\title{
Coherence cost for violating conservation laws
}

\author{
Hiroyasu Tajima, ${ }^{1}$ Naoto Shiraishi, ${ }^{2}$ and Keiji Saito ${ }^{3}$ \\ ${ }^{1}$ Graduate School of Informatics and Engineering, The University of Electro-Communications, 1-5-1 Chofugaoka, Chofu, \\ Tokyo 182-8585, Japan \\ ${ }^{2}$ Department of Physics, Gakushuin University, 1-5-1 Mejiro, Toshima-ku, Tokyo 171-8588, Japan \\ ${ }^{3}$ Department of Physics, Keio University, 3-14-1 Hiyoshi, Yokohama 223-8522, Japan
}

(Received 22 July 2019; revised 25 August 2020; accepted 27 November 2020; published 15 December 2020)

\begin{abstract}
In isolated quantum systems that have conserved quantities, such as energy and total magnetization, the conserved quantities cannot vary their values with their own dynamics. To violate conservation laws of the system keeping the unitary nature in the system's dynamics, quantum coherence must be supplied from the outer environment. This provides a general and abstract interpretation of the underlying mechanism of unitary operations on systems of interest. In this paper, we consider the amount of quantum coherence cost to realize the desired unitary operation. We derive upper and lower bounds for the coherence cost to implement arbitrary unitary operation within the desired error. These two bounds asymptotically match each other in a small error regime, and give an asymptotic equality. We present applications of our theories to several physical situations such as quantum heat engines and entanglement erasure.
\end{abstract}

DOI: 10.1103/PhysRevResearch.2.043374

\section{INTRODUCTION}

Conservation laws indicate that conserved quantities do not change their values with the unitary dynamics of isolated quantum systems. To violate the conservation law without breaking the unitary nature in dynamics, some aid from the outer environments is necessary. Time-dependent parameter control to the system is regarded as a physical phenomenon where the experimental apparatus acts as outer environment. Conceptually, one can interpret this phenomenon as the violation of conservation law by supplying the quantum coherence from the outer environment. A tractable example that has been studied in this context is the realization of the bit flip unitary under the energy conservation law. To realize a bit flip unitary between the ground and excited states on a qubit, an ancillary quantum system which works as an "energy reservoir" is required. Åberg showed that if the ancillary has no quantum coherence, it is impossible to implement the bit flip with high accuracy [1]. The conservation laws restricts the implementation whereas quantum coherence supplied from the outer environment alleviates the restriction.

There have been two main streams of studies regarding this topic. The first type is categorized into studies focusing on sufficient conditions for ancillary coherence to overcome conservation laws and to achieve desired and specific unitary dynamics. These studies mainly aim to coherently control atoms via coherent light [2-9]. These results were further generalized by Åberg in a general framework [1]. This framework clarified what type of quantum superposition is sufficient to

Published by the American Physical Society under the terms of the Creative Commons Attribution 4.0 International license. Further distribution of this work must maintain attribution to the author(s) and the published article's title, journal citation, and DOI. achieve arbitrary unitary operation with arbitrary precision under the conservation law. This idea is applied to several fields such as clocks [10] and heat engines [11]. The second type of research is categorized into studies to clarify necessary conditions for ancillary coherence to overcome the restrictions of the conservation laws [12-16]. This direction of study was initiated by Ozawa who studied the implementation of the controlled-NOT (CNOT) gate [12]. Subsequently, after several researches [13-15], a general lower bound of the required coherence for implementing an arbitrary unitary gate has been obtained [16].

In this paper, we unify these independent studies by providing necessary and sufficient amount of coherence to realize an arbitrary unitary operation. Following previous researches [1,10-16], we regard the unitary operation in the target system as partial dynamics in the composite system. Here, the composite system consists of a target system and an external system (external apparatus) that operates as a "coherence battery." We assume that total dynamics satisfies the conservation law of some physical quantity which is denoted by $A$. (See the schematic in Fig. 1.) We derive upper and lower bounds for the coherence cost, the necessary and sufficient amount of quantum coherence in the battery to achieve unitary time evolution in the target system within the desired error. The upper and lower bounds always converge in the small error limit, and reach an asymptotic equality on the coherence cost. The precise cost can be expressed by only two quantities: implementation error and degree of violation of the conservation law of the desired unitary operation. Furthermore, we derive lower and upper bounds for the coherence cost of nonunitary gates. These bounds show that the tradeoff relations between the coherence cost and the accuracy of operations under conservation laws exist for a broader class of quantum operations than unitary dynamics.

Our result is also significantly related to "channel implementation cost," that is essential in resource theories. In 


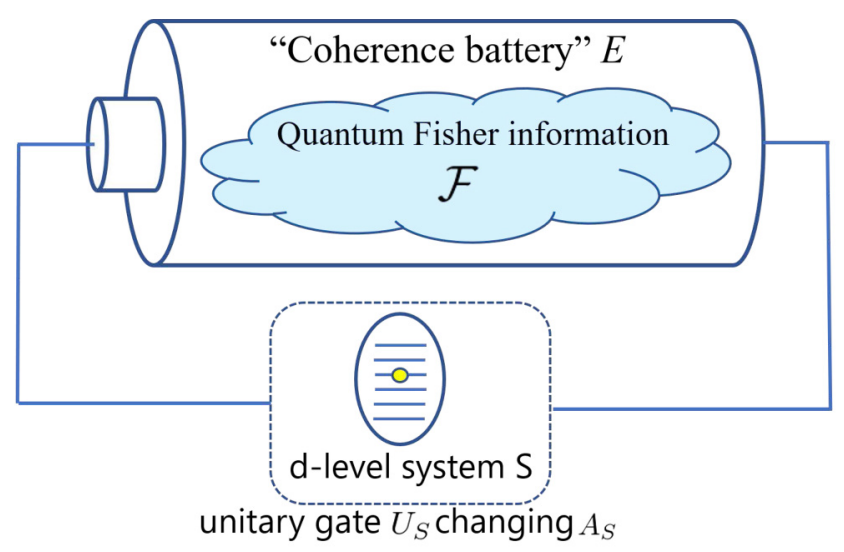

How much $\mathcal{F}$ is "necessary and sufficient"

to implement $U_{S}$ within error $\delta$, under conservation law of $A$ ?

FIG. 1. Schematic of implementation of the unitary operation under the conservation law of physical quantity $A$ (e.g., energy). We aim to implement a unitary operation in the system $S$ that does not conserve the quantity $A_{S}$. Therefore, we attach an external system $E$ that operates as a coherence battery to the system. Although we do not assume that the composite system $S E$ is isolated, we assume that the dynamics of the total system including the environment satisfies the conservation law of $A$, and the environment does not have quantum fluctuation of $A$, i.e., the environment is classical with respect to $A$. In experimental situations, an experimental apparatus that induces the time-dependent Hamiltonian of the system is expected to serve as $E$. We aim to estimate the amount of quantum coherence (measured by the quantum Fisher information) in $E$ required to implement the desired unitary operation on $S$. We present a resource theoretic formulation that expresses this situation (see Fig. 2).

resource theories, we classify some operations and states that can be easily performed and prepared as free, and some states that cannot be created from free states by free operations as resource. Then, operations that are not realizable with only free operations and free states are often possible by using resource states. One of the main subjects in resource theories is to estimate the implementation cost, i.e., the amount of resource required to implement the desired operation by free operations [17]. This problem has been extensively studied for various operations [18-23], such as quantum thermodynamics [18,19], resource erasure [20], and resource theory of coherence [21]. However, the implementation cost in the resource theory of asymmetry [24-35], which considers operations under conservation laws, is not fully understood. Our result fits this category when the implemented channel is unitary and the symmetry is $\mathrm{U}(1)$. In our framework, unitary operation is implemented by combining resource states and covariant operations, which are free operations in the resource theory of asymmetry. We use the quantum Fisher information [36] as a measure of coherence that also serves as a resource measure of the resource theory of asymmetry. Therefore, our results can be interpreted in terms of resource cost for implementing nonfree unitary operations by free operations.

This paper is organized as follows. In Sec. II, we explain the setup and define the quantity of coherence, error of the desired operation on the system, and asymmetry which measures the degree of conservation law violations. In Sec. III, we show the main results of the relations between several quantities defined in Sec. II. In Sec. IV, we discuss the underlying physics of several quantum manipulations. In Secs. V and VI, we briefly present the proof of the main results in Sec. II. Finally, conclusions are presented in Sec. VII.

\section{MOTIVATION AND FORMULATION}

We consider a quantum system $S$. We assume that the system contains a finite-dimensional Hilbert space $\mathcal{H}_{S}$. Suppose that the system comprises a conserved quantity that commutes with the system Hamiltonian. We consider a conserved quantity $A$ and denote its operator by $A_{S}$. Here, $A$ is used as a symbol to denote the conserved quantity, such as energy and total magnetization.

We consider the mechanism to implement the desired unitary operation on the target system $S$ which does not commute with the conserved quantity $A_{S}$. Since time evolution of an isolated system driven by a static system Hamiltonian cannot be such a noncommutative unitary operation, an external resource is necessary. Thus, we attach an external system $E$ that operates as a coherence battery for the system. See the schematic in Fig. 1. As shown later, our theory does not require that the total composite system is completely isolated. We show our results in the most general setup where the composite system is dissipative, being attached to an additional environment. The assumptions that we make are that dynamics of the total system including the additional environment satisfies the conservation law of $A$, and the environment does not have quantum fluctuation on $A$. In experimental situations, an experimental apparatus that induces the time-dependent Hamiltonian of the system is expected to serve as the external system $E$.

The above general setting is expressed in a concise manner using the language of the resource theory of asymmetry [24-35], which is a branch of resource theory that considers operations under conservation laws. In the subsequent subsection, we briefly explain the framework of the resource theory of asymmetry, and, thereafter, more concrete formulation of the above physical situation will be presented. We address the following problem: What is the fundamental limitation to implement unitary dynamics violating the conservation law using the resource stored in the external system? In our formulation, the required resource is measured by the quantum Fisher information, which is a well-known resource measure in the resource theory of asymmetry. Using the measure, we clarify the necessary and sufficient amount of the required resource to implement a given unitary operation. Because the quantum Fisher information is well known as a measure of the quantum fluctuation (i.e., quantum coherence), our result will also clarify the relation between the amount of coherence in the external system and implementation error of the unitary operation.

\section{A. Preliminary: Symmetric state, covariant operation, and quantum Fisher information}

In this subsection, we introduce the basic notions of the resource theory of asymmetry. We introduce symmetric states, covariant operations, and the quantum Fisher information, 
which are, respectively, free states, free operations, and one of the resource measures in the framework of the resource theory of asymmetry. We here explain these using a general quantum system $Q$. In the subsequent sections, the formulation made for the general system $Q$ here is applied to the specific systems $S, E$, or $S E$. We consider a Hermitian operator $A_{Q}$ on the system $Q$ as a conserved quantity in $Q$. A quantum state $\rho_{Q}$ of $Q$ is symmetric with respect to $A_{Q}$ when $\rho_{Q}$ satisfies the following condition:

$$
\mathcal{U}_{Q}(t)\left[\rho_{Q}\right]=\rho_{Q}, \quad \forall t \in \mathcal{R},
$$

where $\mathcal{U}_{Q}(t)[\rho]:=e^{-i A_{Q} t} \rho e^{i A_{Q} t}$ and $\mathcal{R}$ is the total set of real numbers. Note that Eq. (1) holds if and only if the state $\rho_{Q}$ is block-diagonal, or incoherent relative to the eigenspaces of $A_{Q}$, i.e., $\left[A_{Q}, \rho_{Q}\right]=0$. That is, symmetric states are states without quantum fluctuation of $A_{Q}$ because it does not have superposition between the eigenvectors with different eigenvalues.

We next explain the covariant operations. To this end, we introduce invariant unitary operations that are in a special class of the covariant operations. Unitary dynamics $\mathcal{V}_{Q}[\rho]:=$ $V_{Q} \rho V_{Q}^{\dagger}$ is invariant with respect to $A_{Q}$ if and only if the unitary operator conserves $A_{Q}$ :

$$
\left[V_{Q}, A_{Q}\right]=0 .
$$

The name invariance is given because the invariant unitary is invariant with respect to the unitary time evolution generated by $A_{Q}$ :

$$
\mathcal{V}_{Q} \circ \mathcal{U}_{Q}(t)[\ldots]=\mathcal{U}_{Q}(t) \circ \mathcal{V}_{Q}[\ldots], \quad \forall t \in \mathcal{R} .
$$

By generalizing this framework to the completely positive and trace-preserving (CPTP) map, we define the covariant operation. We say that a CPTP map $\Lambda_{Q}$ is covariant with respect to $A_{Q}$ if and only if the map satisfies the covariance condition

$$
\Lambda_{Q} \circ \mathcal{U}_{Q}(t)[\ldots]=\mathcal{U}_{Q}(t) \circ \Lambda_{Q}[\ldots], \quad \forall t \in \mathcal{R} .
$$

It is known that a CPTP map is covariant if and only if the map can be realized by combining invariant unitary operation and symmetric state [28,37]. In other words, the covariance condition (4) is equivalent to the existence of an additional quantum system $Q^{\prime}$, a Hermitian operator $A_{Q^{\prime}}$ on $Q^{\prime}$, a symmetric state $\rho_{Q^{\prime}}$ with respect to $A_{Q^{\prime}}$, and an invariant unitary $V_{Q Q^{\prime}}$ with respect to $A_{Q}+A_{Q^{\prime}}$ on the composite system $Q Q^{\prime}$ satisfying the following relation:

$$
\Lambda_{Q}[\ldots]=\operatorname{Tr}_{Q^{\prime}}\left[V_{Q Q^{\prime}}\left(\ldots \otimes \rho_{Q^{\prime}}\right) V_{Q Q^{\prime}}^{\dagger}\right] .
$$

Note that $\rho_{Q^{\prime}}$ are the incoherent (i.e., classical) state with respect to $A_{Q^{\prime}}$ and $V_{Q Q^{\prime}}$ is the interaction between $Q$ and the additional system $Q^{\prime}$ satisfying the conservation law of $A_{Q}+A_{Q^{\prime}}$. Therefore, we interpret any covariant operation as an open dynamics under the conservation law the environment of which is classical in view of the conserved quantity. In Sec. V (the proof section), the above equivalence between covariant condition (4) and existence of $Q^{\prime}, A_{Q^{\prime}}$, and $V_{Q Q^{\prime}}$ satisfying (5) is used to show that the coherence cost of unitary implementation for the case where $S E$ is isolated is the same as that of the case where $S E$ is not isolated simultaneously. (For details, see the beginning of Sec. V.)
Symmetric states and covariant operations are free states and free operations in the resource theory of asymmetry. Particularly, from (1) and (4), we note that a symmetric state cannot be transformed into a nonsymmetric state by covariant operations. In resource theories, we refer to such nonfree states that we cannot create from free states and free operations as resource states, and evaluate the amount of resource in the resource states by using resource measures. In the resource theory of asymmetry, the quantum Fisher information [36] with respect to $A_{Q}$ is a well-known resource measure [32-35] defined as

$$
\mathcal{F}_{\rho}\left(A_{Q}\right)=2 \sum_{a, b} \frac{\left(p_{a}-p_{b}\right)^{2}}{p_{a}+p_{b}}\left|A_{a b}\right|^{2}
$$

for a given state $\rho$. Herein, $p_{a}$ is the $a$ th eigenvalue of the density matrix $\rho$ with the eigenvector $\psi_{a}$, and $A_{a b}:=\left\langle\psi_{a}\left|A_{Q}\right| \psi_{b}\right\rangle$. The quantum Fisher information satisfies several desirable features as a resource measure in the resource theory of asymmetry. For example, it does not increase through the covariant operation, and it is zero if and only if $\rho$ is symmetric with respect to $A_{Q}$ [32]. Roughly speaking, the quantum Fisher information $\mathcal{F}_{\rho}\left(A_{Q}\right)$ represents the asymptotic conversion rate from $e$-bit state $|\psi\rangle=(|0\rangle+|1\rangle) / \sqrt{2}$ to the state $\rho$ by the covariant operation with respect to $A_{Q}$ [35]. Namely, the quantum Fisher information plays a significantly similar role as the entanglement cost [38] in the entanglement theory. Hence, it can be used to evaluate the cost of the state formation via covariant operations.

The quantum Fisher information is also known as a measure of quantum coherence [35,36,39-41], which satisfies the following relation $[39,40]$ :

$$
\mathcal{F}_{\rho}\left(A_{Q}\right):=\min _{\left\{q_{j}, \phi_{j}\right\}: \rho=\sum_{j} q_{j} \phi_{j}} 4 \sum_{j} q_{j} V_{\phi_{j}}^{2}\left(A_{Q}\right) .
$$

Here the minimization is taken over all possible decompositions $\left\{q_{j},\left|\phi_{j}\right\rangle\right\}$ of the given density matrix $\rho$, and $V_{\rho}\left(A_{Q}\right)$ is the standard deviation of the quantity $A_{Q}$ for the pure state $\left|\phi_{j}\right\rangle$, i.e.,

$$
V_{\phi_{j}}\left(A_{Q}\right):=\sqrt{\left\langle\phi_{j}\left|A_{Q}^{2}\right| \phi_{j}\right\rangle-\left\langle\phi_{j}\left|A_{Q}\right| \phi_{j}\right\rangle^{2}} .
$$

If the decomposition $\phi_{j}$ for the density matrix is identical to the eigenstates of $A_{Q}$ (i.e., $\rho$ is symmetric), the Fisher information $\mathcal{F}$ is exactly zero. Note that in this case, the origin of the fluctuation of $A_{Q}$ in the state $\rho$ is the classical probability $\left\{q_{j}\right\}$. The Fisher information $\mathcal{F}$ takes a nonzero value if $\left|\phi_{j}\right\rangle$ is a superposition of eigenstates with different eigenvalues of $A_{Q}$. In particular, if $\rho$ is a pure state given by $|\phi\rangle\langle\phi|$, the quantum Fisher information is equal to $4 V_{\phi}^{2}\left(A_{Q}\right)$.

\section{B. Implementation of unitary operations under conservation law}

Now, we formulate the implementation of unitary operations on the target system $S$ using the resource in the external system $E$ under the conservation law of the physical quantity $A$. In resource theory of asymmetry, this situation corresponds to the implementation of a nonfree unitary operation on $S$ by combining a free operation on $S E$ and a resource state on $E$ (see Fig. 2). 


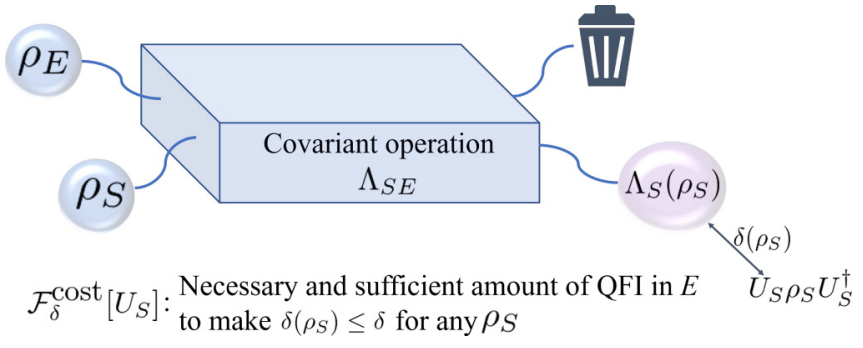

FIG. 2. Schematic of the resource theoretic formulation for implementing the unitary operation under the conservation law of $A$. This formulation precisely corresponds to the situation explained in Fig. 1. We consider the target system $S$ and the external coherence battery $E$, and denote the Hermitian operator of $A$ on $S$ and $E$ by $A_{S}$ and $A_{E}$, respectively. We aim to implement the desired unitary operation $U_{S}$ on $S$ by combining the covariant operation on $S E$ and the initial nonsymmetric state $\rho_{E}$ on $E$. Our purpose is to evaluate the necessary and sufficient amount of the quantum Fisher information (QFI) $\mathcal{F}_{\rho_{E}}\left(A_{E}\right)$ to implement $U_{S}$ within error $\delta$. Because covariant operations and the quantum Fisher information are free operations and a resource measure in the resource theory of asymmetry, we aim to clarify the necessary and sufficient amount of resource to implement the desired unitary operation in resource theory of asymmetry.

We denote the Hilbert space of $E$ by $\mathcal{H}_{E}$. We assume that there also exists a Hermitian operator of the conserved quantity $A$ on $E$ denoted by $A_{E}$. We do not assume that the composite system $S E$ is isolated, and use the CPTP map satisfying the conservation law for the composite system. Dynamics of the composite system $S E$ becomes a covariant operation with respect to the conserved quantity $A_{S}+A_{E}$. We refer to this dynamics as $\Lambda_{S E}$ [42]. We also define the CPTP map of the target system $\Lambda_{S}$ as follows:

$$
\Lambda_{S}\left(\rho_{S}\right):=\operatorname{Tr}_{E}\left[\Lambda_{S E}\left(\rho_{S} \otimes \rho_{E}\right)\right],
$$

where $\rho_{S}$ and $\rho_{E}$ are initial states of the target system and the external system, respectively.

We implement a unitary operation $U_{S}$ that violates the conservation law in the target system. The implementation of $U_{S}$ is fully determined by the set

$$
\mathcal{I}:=\left(\mathcal{H}_{E}, A_{E}, \rho_{E}, \Lambda_{S E}\right),
$$

called the implementation set for the unitary operation. If $\Lambda_{S}\left(\rho_{S}\right)$ approximates $U_{S} \rho_{S} U_{S}^{\dagger}$ accurately for any initial density matrix $\rho_{S}$, we say that the set $\mathcal{I}$ is a good implementation set.

We need to quantify the accuracy of the implementation. To this end, we first introduce a measure of the error in the implementation of the desired unitary dynamics by using the entanglement Bures distance [43]:

$$
\begin{gathered}
L_{e}\left(\rho_{S}, \Lambda\right):=\sqrt{2\left[1-F_{e}\left(\rho_{S}, \Lambda\right)\right]}, \\
F_{e}\left(\rho_{S}, \Lambda\right):=\sqrt{\left\langle\left.\psi\right|_{S R}\left[1_{R} \otimes \Lambda\right]\left(\psi_{S R}\right) \mid \psi\right\rangle_{S R}},
\end{gathered}
$$

where $|\psi\rangle_{S R}$ is a purification of $\rho_{S}$. The symbol $R$ denotes the reference system. The symbol $\psi_{S R}$ is an abbreviation of $\left|\psi_{S R}\right\rangle\left\langle\psi_{S R}\right|$ that will be frequently used in this paper. The operator $\Lambda$ is an arbitrary time-evolution operator that acts only on the Hilbert space of the target system. The entanglement fidelity $F_{e}\left(\rho_{S}, \Lambda\right)$ provides an amplitude of the overlap between the initial state and the final state driven by the time-evolution operator $\Lambda$. The entanglement Bures distance $L_{e}\left(\rho_{S}, \Lambda\right)$ quantifies the distance between these two states. For this general setup of the Bures distance, we next set the time evolution as $\Lambda=\Lambda_{U_{s}^{\dagger}} \circ \Lambda_{S}$, where $\Lambda_{S}$ is the CPTP map defined in Eq. (9) and $\Lambda_{U_{S}^{\dagger}}^{\dagger}$ is an inverse time evolution of the desired unitary dynamics, i.e.,

$$
\Lambda_{U_{S}^{\dagger}}\left(\rho_{S}\right):=U_{S}^{\dagger} \rho_{S} U_{S} .
$$

The operator $\Lambda$ is a successive application of these two maps. With this setup, we can quantify the error by the Bures distance between the final state driven by the desired unitary time evolution and the actual final state. We write this error as a function of the initial state $\rho_{S}$ as

$$
\delta\left(\rho_{S}\right):=L_{e}\left(\rho_{S}, \Lambda_{U_{S}^{\dagger}} \circ \Lambda_{S}\right) .
$$

In addition, we define the error of implementation as the worst case over all initial states:

$$
\delta_{\mathcal{I}}:=\max _{\rho_{S}} \delta\left(\rho_{S}\right) .
$$

If the error $\delta_{\mathcal{I}}$ of an implementation set $\mathcal{I}$ is less than a value $\delta$, we say that the implementation set $\mathcal{I}$ realizes $U_{S}$ within error $\delta$.

As already discussed, we can regard the quantum Fisher information as an amount of coherence (or resource). We here define the amount of coherence cost that the external system must bear to achieve the desired unitary operation in the system. We consider the situation wherein the desired unitary operation is achieved within the error $\delta$. We define the coherence cost $\mathcal{F}_{\delta}^{\text {cost }}\left[U_{S}\right]$ as the minimal value of the quantum coherence over all possible implementation sets that implements the desired unitary operation within error $\delta$ :

$$
\mathcal{F}_{\delta}^{\text {cost }}\left[U_{S}\right]:=\min _{\mathcal{I}: \delta_{\mathcal{I}} \leqslant \delta} \mathcal{F}_{\rho_{E}}\left(A_{E}\right) .
$$

We finally define the degree of asymmetry of the desired unitary $U_{S}$. The asymmetry in the present context implies a degree of violating the conservation law inside the target system by the unitary operation $U_{S}$. We quantify this through the amount of noncommutativity between $U_{S}$ and $A_{S}$ :

$$
\mathcal{A}_{U_{S}}:=\frac{\Delta_{A_{S}^{\prime}-A_{S}}}{2},
$$

where $A_{S}^{\prime}:=U_{S}^{\dagger} A_{S} U_{S}$, and $\Delta_{X}$ is the absolute value of the difference between maximum and minimum eigenvalues of the operator $X$, respectively. Note that the maximum (minimum) eigenvalue of $A_{S}^{\prime}-A_{S}$ is the maximum (minimum) value of the difference between expectation values of $A_{S}$ for $\rho_{S}$ and $U_{S} \rho_{S} U_{S}^{\dagger}$. Therefore, the quantity $\mathcal{A}_{U_{S}}$ shows the ability of the desired unitary $U_{S}$ to change the expectation value of the conserved quantity $A_{S}$. This quantity is non-negative, and becomes zero if and only if $U_{S}$ and $A_{S}$ commute with each other [44]. Hence we can interpret that a finite $\mathcal{A}_{U_{S}}$ reflects the violation of the conservation law by unitary operation.

In the next section, we derive an asymptotic equality among $\mathcal{F}_{\delta}^{\text {cost }}\left[U_{S}\right], \delta$, and $\mathcal{A}_{U_{S}}$. It clarifies the necessary and sufficient amount of resource to implement a desired 
nonfree unitary by free operations in the resource theory of asymmetry.

\section{MAIN RESULTS}

\section{A. Coherence cost for unitary operations}

We present the main results of this paper and discuss their physical consequences. The proof of the results will be provided later.

Theorem 1. Let $(\mathcal{F}, \delta)$ be a tuple of positive real numbers satisfying $0 \leqslant \delta \leqslant \sqrt{2}$. If the tuple of positive numbers $(\mathcal{F}, \delta)$ satisfies the following inequality, then there is no implementation set $\mathcal{I}$ satisfying $\delta_{\mathcal{I}} \leqslant \delta$ and $\mathcal{F}_{\rho_{E}}\left(A_{E}\right)=\mathcal{F}$ :

$$
\sqrt{\mathcal{F}}<\frac{\mathcal{A}_{U_{S}}}{\delta}-6 \Delta_{A_{S}}
$$

Theorem 2. Let $(\mathcal{F}, \delta)$ be a tuple of positive real numbers satisfying $0 \leqslant \delta \leqslant 4 \sqrt{2} \mathcal{A}_{U_{s}} /\left(9 \Delta_{A_{s}}\right)$. If the tuple satisfies the following inequality, then there exists an implementation set $\mathcal{I}$ such that $\Lambda_{S E}$ is an invariant unitary operation, $\delta_{\mathcal{I}} \leqslant \delta$ and $\mathcal{F}_{\rho_{E}}\left(A_{E}\right)=\mathcal{F}$ :

$$
\sqrt{\mathcal{F}} \geqslant \frac{\mathcal{A}_{U_{S}}}{\delta}+\sqrt{2} \Delta_{A_{S}}
$$

Theorem 1 presents a necessary condition for the existence of the implementation set. It indicates that to realize $U_{S}$ within error $\delta$, the quantum Fisher information of the implementation device $\mathcal{F}_{\rho_{E}}\left(A_{E}\right)$ should be larger than or equal to $\frac{\mathcal{A}_{U_{S}}}{\delta}-6 \Delta_{A_{S}}$. Theorem 2 shows a sufficient condition for the existence of the implementation set. It guarantees that there exists an implementation device that achieves $U_{S}$ within error $\delta$ and the quantum Fisher information of which is less than or equal to $\frac{\mathcal{A}_{U_{S}}}{\delta}+\sqrt{2} \Delta_{A_{S}}$. From Theorems 1 and 2, we can derive lower and upper bounds for the coherence cost in implementation of $U_{S}$ within error $\delta$ :

$$
\frac{\mathcal{A}_{U_{S}}}{\delta}-6 \Delta_{A_{S}} \leqslant \sqrt{\mathcal{F}_{\delta}^{\text {cost }}\left[U_{S}\right]} \leqslant \frac{\mathcal{A}_{U_{S}}}{\delta}+\sqrt{2} \Delta_{A_{S}},
$$

where the upper bound holds for the region $0 \leqslant \delta \leqslant$ $4 \sqrt{2} \mathcal{A}_{U_{S}} /\left(9 \Delta_{A_{S}}\right)$. We emphasize that we do not impose any conditions on the unitary operation $U_{S}$ and hence the two theorems hold for any desired unitary operation. The desired implementation set, the existence of which is guaranteed by Theorem 2, will be given constructively in Sec. VI.

We numerically demonstrate Theorems 1 and 2 by taking a specific example. We consider $S$ as a qubit, $A_{S}$ as a Hamiltonian $|1\rangle\langle 1|$ (i.e., the energy eigenvalue of $|0\rangle$ is zero), and $U_{S}$ as a bit flip unitary $|0\rangle\langle 1|+| 1\rangle\langle 0|$. Thereafter, the inequality (18) in Theorem 1 draws a regime of the implementation error and the quantum Fisher information that we cannot achieve. We depict the unachievable regime as the region $A$ in Fig. 3. In the region $A$, one cannot implement the desired unitary operation within error $\delta$ by any implementation set $\mathcal{I}$ satisfying $\mathcal{F}_{\rho_{E}}\left(A_{E}\right)=\mathcal{F}$. That is, in the region $A$, the amount of coherence $\mathcal{F}$ is insufficient to implement the desired unitary operation within error $\delta$. Moreover, the inequality (19) in Theorem 2 indicates an achievable region that is shown as the region $B$ in Fig. 3. In the region $B$, we can implement the desired unitary operation within error $\delta$ using an implementation set $\mathcal{I}$ satisfying $\mathcal{F}_{\rho_{E}}\left(A_{E}\right)=\mathcal{F}$. That

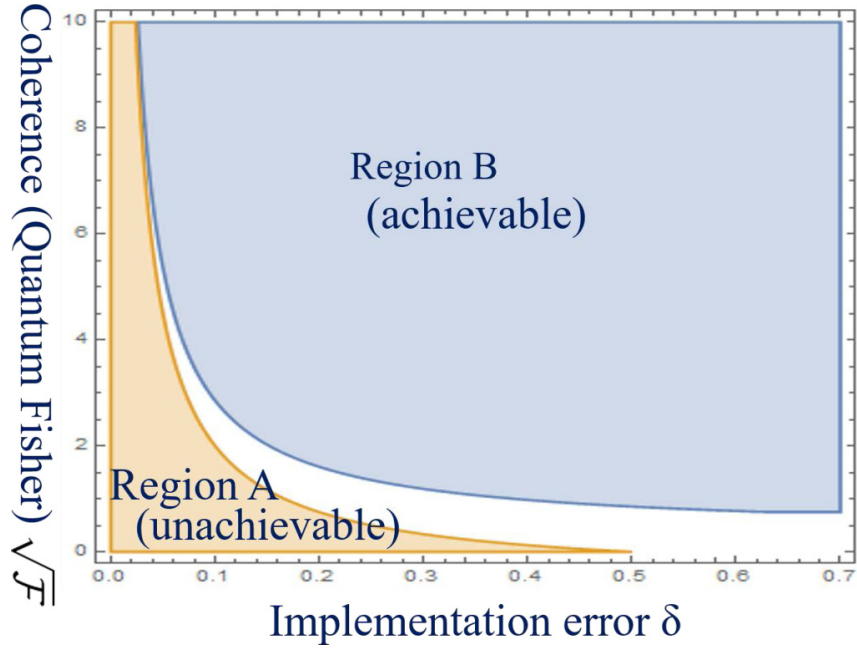

FIG. 3. A graph indicating (18) and (19) for the specific model. The system is a qubit system the Hamiltonian of which is $|1\rangle\langle 1|$ (i.e., the energy eigenvalue of the ground state $|0\rangle$ is zero) and the desired unitary operation is the bit flip unitary $|1\rangle\langle 0|+| 0\rangle\langle 1|$. In the region $A$, no set achieves the desired unitary operation, as indicated from the inequality (18), whereas in the region $B$ at least one set achieves the desired unitary operation.

is, in the region $B$, the amount of coherence $\mathcal{F}$ is sufficient to implement the desired unitary within the error $\delta$ [45].

As illustrated in Fig. 3, the two regions A and B (achievable and unachievable regimes) converge to the same line as the implementation error $\delta$ approaches zero. This implies that the coherence $\operatorname{cost} \mathcal{F}_{\delta}^{\text {cost }}\left[U_{S}\right]$ approaches the bound in Theorem 1. The upper and lower bounds in (20) indicate the following asymptotic relation for the coherence cost:

$$
\sqrt{\mathcal{F}_{\delta}^{\text {cost }}\left[U_{S}\right]}=\frac{\mathcal{A}_{U_{S}}}{\delta}+O(1)
$$

where $O$ is Bachmann-Landau notation as $\delta \rightarrow 0$. The asymptotic equality (21) explicitly shows a closed relation among the coherence cost, and the degree of asymmetry and the implementation error of the desired unitary operation. For a fixed asymmetry, a large coherence is necessary for an accurate implementation of the unitary operation. The equation (21) represents the tradeoff relation between them. Also, large asymmetry requires large coherence.

The asymptotic equality (21) shows that the coherence cost quadratically depends on the asymmetry $\mathcal{A}_{U_{S}}$. From the definition (17), the asymmetry $\mathcal{A}_{U_{S}}$ is of the same order as the conserved quantity $A_{S}$. Suppose that the system of interest is a macroscopic system, and the conserved quantities satisfy extensivity with respect to the system size. In this case, the coherence cost for achieving the desired operation must be the square of the system size. Remarkably, this implies that the implementation of unitary operations for macroscopic systems requires significantly large coherence cost.

\section{B. Coherence cost for restricted initial states}

In the previous subsection, we established an asymptotically tight relation among the coherence cost, error of the unitary operation, and amount of asymmetry. Note that in 
the previous subsection, we have quantified the error as that of the worst case over all possible initial states. However, sometimes we are interested in the implementation of desired unitary dynamics for specific initial states. In this case, what conditions must be imposed on the coherence cost in external systems? We herein address this question.

Let $U_{S}$ be a transformation providing the desired final state from the initial state $\rho_{S}$. We herein discuss the properties of transformation on the implementation set $\mathcal{I}$. For the goal, we take reference state $\left\{\psi_{i}\right\}$ that is an orthonormal basis of the system satisfying $r_{i}:=\left\langle\psi_{i}\left|\rho_{S}\right| \psi_{i}\right\rangle<1$ for all $i$. We have the following inequality that presents a necessary condition for the implementation set similar to Theorem 1:

$$
\sqrt{\mathcal{F}_{\rho_{E}}\left(A_{E}\right)} \geqslant \frac{\chi\left(\rho_{S},\left\{\psi_{i}\right\}\right)}{5 \sqrt{\delta\left(\rho_{S}\right)^{2}+\sum_{i} r_{i} \delta\left(\psi_{i}\right)^{2}}}-4 \Delta_{A_{S}},
$$

where $A_{S}^{\prime}:=U_{S}^{\dagger} A_{S} U_{S}$, and $r_{i}:=\left\langle\psi_{i}\left|\rho_{S}\right| \psi_{i}\right\rangle$ quantifies the weight of $\left|\psi_{i}\right\rangle$ in $\rho_{S}$. In the above, $\chi$ is defined as the fluctuation of the change in $A_{S}$ with respect to the reference states $\left\{\psi_{i}\right\}$ as follows:

$$
\chi\left(\rho_{S},\left\{\psi_{i}\right\}\right):=\sqrt{\sum_{i} r_{i}\left(\left\langle A_{S}^{\prime}-A_{S}\right\rangle_{\psi_{i}}-\left\langle A_{S}^{\prime}-A_{S}\right\rangle_{\rho_{S}}\right)^{2}},
$$

where $\langle\ldots\rangle_{\psi_{i}}=\operatorname{tr}\left(\ldots\left|\psi_{i}\right\rangle\left\langle\psi_{i}\right|\right)$ and $\langle\ldots\rangle_{\rho_{S}}=\operatorname{tr}\left(\ldots \rho_{S}\right)$.

The inequality (22) shows that for various nonunitary CPTP maps, much coherence is necessary to implement the CPTP map within small error. In general, the inequality (22) guarantees that there is a tradeoff relation between the error and the coherence cost for an arbitrary implementation of an arbitrary CPTP map $\mathcal{E}$ satisfying the following three conditions [46].

(1) $\mathcal{E}$ acts as unitary dynamics $U_{S}$ for a certain initial state $\rho_{S}$.

(2) $\mathcal{E}$ acts as unitary dynamics $U_{S}$ for the orthonormal states $\left\{\psi_{k}\right\}_{k \in \mathcal{K}}$ that span the support of $\rho_{S}$

(3) For states $\psi_{i}$ satisfying $r_{i}>0$, at least one of $\left\{\left\langle A_{S}-A_{S}^{\prime}\right\rangle_{\psi_{k}}\right\}_{k \in \mathcal{K}}$ has a different value from others.

A typical example of CPTP maps satisfying the above three conditions is a CPTP map that acts as an asymmetric unitary for a subspace of the Hilbert space of $S$. To illustrate this, we consider the gate on a $d$-level system $S$ that behaves as a bit flip unitary only for the space spanned by the ground state and the first excited state but behaves as a nonunitary gate for other states. Because this is not a unitary operation for the entire Hilbert space, we cannot apply Theorem 1. However, the inequality (22) is available to estimate the required coherence cost for implementing this nonunitary gate within error $\delta$ under the energy conservation law. We examine how (22) works for this bit flip example. We refer to the ground state and the first excited state as $\psi_{0}$ and $\psi_{1}$, respectively. We also define the energy eigenvalues of $\psi_{0}$ and $\psi_{1}$ as $E_{0}$ and $E_{1}$, respectively. Let $\rho_{S}$ be defined by $\rho_{S}=\frac{\psi_{0}+\psi_{1}}{2}$. Then, $\chi\left(\rho_{S},\left\{\psi_{i}\right\}\right)=\sqrt{\left(E_{0}-E_{1}\right)^{2} / 2}, \delta\left(\rho_{S}\right) \leqslant \delta$, and $\delta\left(\psi_{k}\right) \leqslant \delta$ for $k=0,1$ hold. Because $r_{k}=0$ holds for all $k \geqslant 2$ in this case, $\sqrt{\delta\left(\rho_{S}\right)^{2}+\sum_{i} r_{i} \delta\left(\psi_{i}\right)^{2}} \leqslant \sqrt{2} \delta$ holds. Therefore, the inequality (22) guarantees that the necessary coherence is larger than or equal to $\left|E_{0}-E_{1}\right| / 10 \delta-4\left\|H_{S}\right\|$, where $H_{S}$ is the Hamiltonian of the system $S$.

\section{APPLICATION}

In this section, based on the relations (21) and (22), we discuss the underlying physical mechanisms in manipulating quantum states. For several cases, special attention is paid to the coherence cost that must be prepared in the external system. The cases in subsections A and B are discussed with the relation (21) and the case in subsection $\mathrm{C}$ is discussed with the relation $(22)$.

\section{A. Underlying physics to implement the time-dependent Hamiltonian}

Our results clarify the coherence cost for realizing unitary dynamics that changes the conserved quantity. Since energy is a conserved quantity, our theory is of course applicable to the implementation the following unitary dynamics $U_{S}$, which is caused by a time-dependent Hamiltonian $\tilde{H}_{S}(t)$ that changes the energy inside the system:

$$
U_{S}:=\mathcal{T} \exp \left(-i \int_{0}^{\tau} d t \tilde{H}_{S}(t)\right),
$$

where $\mathcal{T}$ implies the time-ordered operator.

As an example, let us consider the Jaynes-Cummings (JC) model. In the JC model, we consider a two-level atom interacting with the electromagnetic field. The Hamiltonians are written as

$$
H_{S}=\epsilon \sigma_{z}, H_{S E}=\lambda\left(\sigma_{+} a+a^{\dagger} \sigma_{-}\right), H_{E}=2 \epsilon a^{\dagger} a,
$$

where $\lambda$ is the amplitude of the interaction, and the operator $\sigma_{z}$ is the $z$ component of the Pauli matrix. The operator $\sigma_{+}$ $\left(\sigma_{-}\right)$flips the $z$ spin from down (up) to up (down), and the operators $a^{\dagger}$ and $a$ are, respectively, the creation and annihilation operators of the photon. In the JC model, the coherent light approximately induces the time-dependent Hamiltonian on the atom that changes the energy. By setting $A_{S}$ and $A_{E}$ in Theorems 1 and 2 to $H_{S}$ and $H_{E}$, we figure out the fundamental bounds for the amount of coherence of the coherent light that is necessary to realize the desired time-dependent Hamiltonian on the two-level atom. Let us consider the case where the initial state of the electromagnetic field is the coherent state:

$$
\rho_{E}=|\alpha\rangle\langle\alpha|, \quad| \alpha\rangle=e^{\alpha\left(a^{\dagger}-a\right)}|0\rangle,
$$

where $|0\rangle$ is the vacuum state, and the parameter $\alpha$ is a real number. If we take the limit of $\lambda \rightarrow+0$ setting $\lambda \alpha$ to a constant, the reduced dynamics converges to the unitary time evolution (24), where the Hamiltonian $\tilde{H}_{S}(t)$ is

$$
\tilde{H}_{S}(t)=\epsilon \sigma_{z}+\lambda \alpha\left(\sigma_{+} e^{-i 2 \epsilon t}+\sigma_{-} e^{i 2 \epsilon t}\right) .
$$

If we do not take the above limitation, namely, if we take a finite $\lambda$, there is an error between the reduced dynamics of the two-level atom and the unitary dynamics $U_{S}$. Our results clarify the tradeoff relation between the amount of coherence in the external system (in this case, the external electromagnetic field) and the error of realization of the time-dependent Hamiltonian [in this case (27)] on the target system. 
From the above example, our results show that to realize the time-dependent Hamiltonian accurately, we need to prepare much quantum fluctuation in the external system. This fact gives a resource-theoretic interpretation for the so-called classicalization of external systems. When we implement a time-dependent Hamiltonian, we usually treat the external system as a classical system. Our results indicate that to treat the external system as a classical system, the external system must have much coherence. In the implementation of the time-dependent Hamiltonian using an external apparatus, the apparatus typically uses the classical electromagnetic interaction with the target system. And the classical electromagnetic fields are obtained in the limit of large amplitude of the coherent state. Therefore, it is consistent with the present theory.

Since a classical electromagnetic field has a uniquely determined value, it is important to understand how the above large quantum fluctuations and the classicalization are consistent. Let us consider the above JC model again. If the reduced dynamics of the atom $(=S)$ changes its energy, the amount of change must be compensated by the energy in the electromagnetic field $(=E)$. Moreover, to make the reduced dynamics close to unitary, the state in the external system should not be damaged by exchanging energy between $S$ and $E$. This is possible if the external state has a large variance with respect to the energy scale of $S$ [47], while the variance is small with respect to the energy scale of $E$. Therefore, if we realize a time-dependent Hamiltonian on the target system using a "classical" external system, the external system has quantum energy fluctuation much larger than the energy scale of the target system, while fluctuation is negligibly small with respect to the energy scale of the external system itself.

\section{B. Quantum heat engines: Quantum work storage}

In this subsection, we consider the application of our results to quantum cyclic heat engines, which is particularly suggestive. In the analysis of quantum heat engines, a model called the standard model is widely used [48-57]. In the standard model, we consider a composite system of a working body and a heat bath as a target system $S$, and assume that the dynamics of the target system is described by the timedependent Hamiltonian. Namely, the time evolution of the target system is described as

$$
\begin{gathered}
\rho_{S}(t)=U_{S}(t) \rho_{S}(0) U_{S}^{\dagger}(t), \\
U_{S}(t)=\mathcal{T} \exp \left(-i \int_{0}^{t} d s \tilde{H}_{S}[\lambda(s)]\right)
\end{gathered}
$$

where $\lambda(t)$ is a control parameter of the system Hamiltonian, e.g., the strength of the magnetic field and/or the position of a piston, etc. In the standard model, we also assume that the work extracted from the target system is stored in an external work storage (it is often called the "external agent") through the back action of control parameters. Namely, the conservation law of energy in the composite system of the target system and the work storage is assumed. The amount of extracted work is defined by the difference of the expectation value of energy in the target system:

$$
\langle W\rangle:=\operatorname{Tr}\left\{\rho_{S} \tilde{H}_{S}[\lambda(0)]-U_{S} \rho_{S} U_{S}^{\dagger} \tilde{H}_{S}[\lambda(\tau)]\right\} .
$$

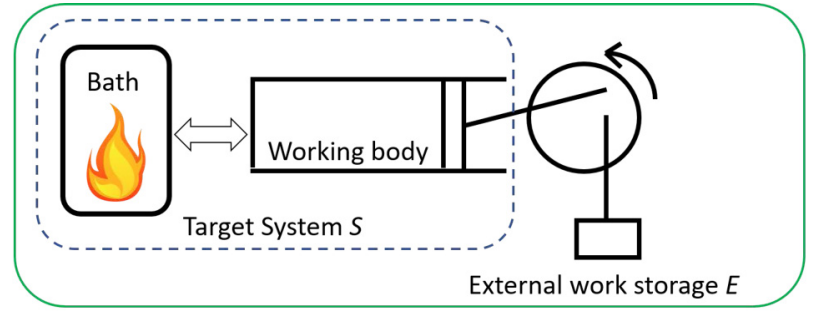

Standard model assumes the dynamics of $?_{---}^{--}$, to be unitary

Quantum thermodynamics assumes the dynamics of $\square$ to be unitary What is the condition for E to obtain standard model from quantum thermodynamic setup?

FIG. 4. Schematic diagram of how we apply our result to the quantum heat engine and work storage. We consider three systems: working body, heat bath, and work storage. In quantum thermodynamics, we consider all of these three as quantum systems, and assume the time evolution of the composite system of these three to be energy-preserving unitary. On the other hand, in the standard model, we consider only the composite system of the working body and the heat bath as a quantum system (that we call "target system"), and assume the time evolution of the target system to be energynonpreserving unitary. The problem is to clarify conditions that work storage must satisfy to obtain a standard model from the quantum thermodynamic setup as a kind of macroscopic limit. Since the total system (the target system and the work storage) satisfies the conservation law of energy, we can apply our theory to clarify conditions. In order for the standard model to be a good approximation, the work storage must contain a much larger fluctuation of energy than the amount of extracted work.

In the standard model, we do not usually treat the external work storage as a quantum system. This treatment of the external work storage goes well with experimental setups, in that the work storage is a classical experimental apparatus that controls parameters [58]. On the other hand, in the framework of purely quantum thermodynamics, the work storage is explicitly treated as a quantum system [11,59-67]. In the quantum thermodynamic setup, dynamics of the composite system of the target system and the work storage is described as an invariant unitary operation, and the work storage stores the work. It is important to understand when and with what condition the setup of the standard model can be justified from the viewpoint of purely quantum thermodynamic setups. Note that purely quantum thermodynamic setup fits our general framework by regarding the work storage as an external system. When the dimension of the Hilbert-space dimension of the bath is large but finite, we can use our theory to clarify what conditions the work storage must satisfy to justify the framework of the standard model (see Fig. 4).

We now apply Theorem 1 to the setup by setting $A_{S}$ to the Hamiltonian of target system $H_{S}$ and $A_{E}$ to the Hamiltonian of work storage. Then, according to Theorem 1, the external system must have sufficiently large energy fluctuations when realizing a time-dependent Hamiltonian. As argued in the previous subsection, for a perfect unitary control on the system, the required energy fluctuation must be large such that the energy gain of the work storage is negligibly small compared to the energy fluctuation of the work storage. 


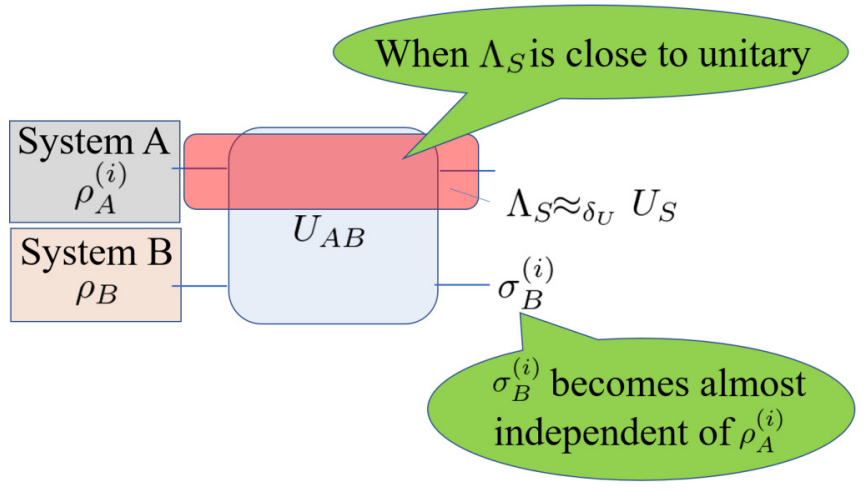

FIG. 5. Schematic diagram of the inequality (40). If the dynamics of the system $A$ is close to unitary, the final state of $B$ is close to independent of the initial state of $A$.

A similar argument to the above also provides an important message regarding the detectability of work of quantum heat engines, that is analyzed in previous results [68,69]. Again, we consider a heat engine that is a composite system of a working body and heat baths, and an external work storage that stores the work extracted from the heat engine. Consider the situation wherein we aim to detect the energy gain in the work storage by measuring the work storage. For example, this situation corresponds to determining the amount of work by comparing the initial and final positions of the weight lifted by the heat engine. Using the tradeoff relation between information gain and disturbance in measurements, previous studies $[68,69]$ showed that if the time evolution of the heat engine can be described in terms of unitary dynamics as assumed by the standard model, the amount of work cannot be detected by measuring the storage. Our result provides an intuitive explanation on why such loss of detectability occurs. As discussed above, the work storage must exhibit a much larger energy fluctuation than the energy gain from the heat engine so that the energy gain of the work storage does not damage the overall energy distribution of the work storage: Thus, we cannot detect the amount of energy gain.

\section{Coherence cost for entanglement erasure}

Our main result (21) provides the coherence cost for the implementation of unitary gates. However, we can also consider the coherence costs for nonunitary state transformations using the relation (22). As a typical example, we apply our results to entanglement erasure. Given an entangled initial state $\alpha|00\rangle+\beta|11\rangle$ with arbitrary $\alpha, \beta$ satisfying $|\alpha|^{2}+|\beta|^{2}=1$, we perform the following entanglement erasure process:

$$
\alpha|00\rangle+\beta|11\rangle \rightarrow \alpha|00\rangle+\beta|10\rangle .
$$

This erasure process might be a nonunitary CPTP operation, since the state transformation is not specified for initial states except for the states written in the form of $\alpha|00\rangle+\beta|11\rangle$. Therefore, the formula (21) cannot evaluate the necessary coherence for the state transition (31). Even for this case, our second main result (22) is still valid and claims that if some devices can perform the state transition (31) within the error $\delta$ for arbitrary $\alpha$, the device must contain much coherence (i.e., quantum Fisher information) in proportion to $1 / \delta^{2}$.
In order to apply our framework to this case, we set the two qubits as the system $S$, and an implementation device as the external system $E$. We assume that the total magnetization, i.e., the amount of $z$ component of the two spins, is conserved. We here write the magnetization as $A_{S}=2|1\rangle\left\langle\left. 1\right|^{\otimes 2}+\right.$ $|1\rangle\langle 1|\otimes| 0\rangle\langle 0|+| 0\rangle\langle 0|\otimes| 1\rangle\langle 1|$. We also assume that an implementation set $\mathcal{I}=\left(\mathcal{H}_{E}, A_{E}, \rho_{E}, U_{S E}\right)$ realizes the state transition (31) within the error $\delta$ for arbitrary $\alpha$, and that the total dynamics $U_{S E}$ satisfies $\left[U_{S E}, A_{S}+A_{E}\right]=0$. Subsequently, for arbitrary $\alpha$ and $\beta$ such that $|\alpha|^{2}+|\beta|^{2}=1$, three inequalities $\delta(|00\rangle) \leqslant \delta, \delta(|11\rangle) \leqslant \delta$ and $\delta(\alpha|00\rangle+\beta|11\rangle) \leqslant$ $\delta$ are satisfied by setting the specific unitary transformation $U_{S}^{\prime}:=|00\rangle\langle 00|+| 01\rangle\langle 01|+| 11\rangle\langle 10|+| 10\rangle\langle 11|$. Note that the states $|00\rangle$ and $|11\rangle$ are the eigenstates of $A_{S}^{\prime \prime}-A_{S}$ with $A_{S}^{\prime \prime}=U_{S}^{\prime \dagger} A_{S} U_{S}^{\prime}$. Substituting the above into (22), we obtain the following inequality for an arbitrary pair of $(\alpha, \beta)$ :

$$
\sqrt{\mathcal{F}_{\rho_{E}}\left(A_{E}\right)} \geqslant \frac{\chi(\alpha|00\rangle+\beta|11\rangle,\{|00\rangle,|11\rangle\})}{5 \sqrt{2} \delta}-4 \Delta_{A_{S}} .
$$

Since we have assumed that $\mathcal{I}$ satisfies $\delta(\alpha|00\rangle+\beta|11\rangle) \leqslant \delta$ for any $(\alpha, \beta)$, the initial state $\rho_{E}$ of $E$ must satisfy (32) for all $(\alpha, \beta)$. Therefore we obtain

$$
\begin{aligned}
\sqrt{\mathcal{F}_{\rho_{E}}\left(A_{E}\right)} & \geqslant \max _{\alpha} \frac{\chi(\alpha|00\rangle+\beta|11\rangle,\{|00\rangle,|11\rangle\})}{5 \sqrt{2} \delta}-4 \Delta_{A_{S}} \\
& \geqslant \frac{1}{5 \sqrt{2} \delta}-4 \Delta_{A_{S}} .
\end{aligned}
$$

In the second inequality, we used the fact that the maximum value of $\chi(\alpha|00\rangle+\beta|11\rangle,\{|00\rangle,|11\rangle\})$ is 1 . In this derivation, we only assume that the CPTP map implemented by $\mathcal{I}$ is closed to $U_{S}^{\prime}$ only for the special initial states $\alpha|00\rangle+\beta|11\rangle$. Even when the CPTP map given by $\mathcal{I}$ is far from unitary for other initial states, inequality (33) holds. The inequality (33) demonstrates that a large coherence is required for entanglement erasure, even considering the implementation of gates that are not unitary gates.

\section{DERIVATION OF LOWER BOUNDS OF COHERENCE COST}

Here, we derive the lower bounds of coherence cost, i.e., Theorem 1 and the inequality (22). We initially show that it is enough to show the case that $\Lambda_{S E}$ is an invariant unitary, since a covariant operation can always be realized by combining an invariant unitary and a symmetric state [28] as we have explained in Sec. II A (note that the quantum Fisher information of a symmetric state is zero and that the quantum Fisher information is additive for a product state). Therefore, in this section, we treat the case where $\Lambda_{S E}$ is invariant unitary dynamics such that $\Lambda_{S E}[\ldots]=U_{S E}[\ldots] U_{S E}^{\dagger}$, where $U_{S E}$ satisfies $\left[U_{S E}, A_{S}+A_{E}\right]=0$. In this case, the expectation value of $A_{S}+A_{E}$ is conserved, and hence in this dynamics the quantity $A_{E}$ must compensate the change in $A_{S}$.

\section{A. Main idea of proof of lower bounds of coherence cost}

Before discussing the proofs of Theorem 1 and (22), we will present the main idea (outline) of these proofs. The key ingredient in these proofs is the following. 
Lemma 1. Consider two quantum states, $\sigma_{1}$ and $\sigma_{2}$, and an observable $X$. We define the difference between the expectation values of $X$ for $\sigma_{1}$ and $\sigma_{2}$ as $\Delta:=\left|\operatorname{Tr}\left[X\left(\sigma_{1}-\sigma_{2}\right)\right]\right|$, and the Bures distance

$$
L\left(\sigma_{1}, \sigma_{2}\right):=\sqrt{2\left(1-\operatorname{Tr}\left[\sqrt{\sqrt{\sigma_{1}} \sigma_{2} \sqrt{\sigma_{1}}}\right]\right)} .
$$

We obtain the following key relation, that was first presented in Ref. [16]:

$$
\Delta \leqslant L\left(\sigma_{1}, \sigma_{2}\right)\left(V_{\sigma_{1}}(X)+V_{\sigma_{2}}(X)+\Delta\right),
$$

where $V_{\sigma}(X)$ is the standard deviation of $\sigma$ with respect to $X$.

The key relation (35) claims that the expectation values of $X$ can differ significantly in two states only if (i) these two states differ significantly, or (ii) at least one of the standard deviations of $X$ in these states is large. To understand the meaning of the condition (ii), we provide an example: Consider $\langle 0|X| 0\rangle=0,\langle x|X| x\rangle=x$, and set $\left|\sigma_{1}\right\rangle=|0\rangle$ and $\left|\sigma_{2}\right\rangle=\sqrt{1-\varepsilon}|0\rangle+\sqrt{\varepsilon}|x\rangle$. The distance between these two states, $L\left(\sigma_{1}, \sigma_{2}\right)$, depends only on $\varepsilon$, not on $x$. Hence, even if $\varepsilon$ is small (i.e., two states are close to each other), $\Delta$ can increase by setting $x$ large. In this case, the standard deviation of $X$ in $\sigma_{2}$ increases with $x$.

In the derivation of the lower bounds for coherence, we use the key relation (35) by setting $X$ as $A_{E}$, the conserved quantity in $E$, and $\sigma_{1}$ and $\sigma_{2}$ as the two final states of $E$ with different initial states of $S$. In addition, we use the following three relations.

(a) If the time evolution of $S$ is well approximated by a unitary operation, then any two final states of $E$ with different initial states of $S$ are close to each other (See Fig. 5).

(b) If the variance of $A_{E}$ for a final state of $E$ is large, then that for the initial state of $E$ is also large.

(c) If the fluctuation of the exchange of the conserved quantity $A$ between $S$ and $E$ is large, then the expectation value of $A_{E}$ for the final state of $E$ largely varies depending on the initial states of $S$.

The relation (a) is given as a consequence of the fact that a very small correlation between $S$ and $E$ is formed when the time evolution of $S$ is close to unitary. To describe this relation quantitatively, we use an important lemma shown in Appendix A. The relations (b) and (c) are given as the consequences of the conservation law $\left[U_{S E}, A_{S}+A_{E}\right]=0$. In deriving Theorem 1 , the relation (a) connects $L\left(\sigma_{1}, \sigma_{2}\right)$ and $\delta$, (b) connects $V_{\sigma_{i}}(X)(i=1,2)$ and $\mathcal{F}_{\rho_{E}}\left(A_{E}\right)$, and (c) connects $\Delta$ and $\mathcal{A}_{U_{S}}$.

\section{B. Proof of Theorem 1}

In this subsection, we explain the proof of Theorem 1. As pointed out at the beginning of this section, it is enough to prove Theorem 1 for the case where $\Lambda_{S E}$ is unitary. We show the following proposition that is the contraposition of Theorem 1.

Proposition 1. Let $\delta$ be a real positive number satisfying $0 \leqslant \delta \leqslant \sqrt{2}$. For any implementation set $\mathcal{I}=$ $\left(\mathcal{H}_{E}, A_{E}, \rho_{E}, U_{S E}\right)$ satisfying $\delta_{\mathcal{I}} \leqslant \delta$, the following inequality holds:

$$
\sqrt{\mathcal{F}_{\rho_{E}}\left(A_{E}\right)} \geqslant \frac{\mathcal{A}_{U_{S}}}{\delta}-6 \Delta_{A_{S}}
$$

To prove this proposition, we introduce some symbols. We prepare three initial states of $S ; \rho_{S, \uparrow}, \rho_{S, \downarrow}$; and $\rho_{S, \uparrow+\downarrow}:=$ $\left(\rho_{S, \uparrow}+\rho_{S, \downarrow}\right) / 2$. The state $\rho_{S, \uparrow}\left(\rho_{S, \downarrow}\right)$ maximizes (minimizes) the loss of the quantity $A$ in the system through the unitary dynamics $U_{S}$ :

$$
\begin{aligned}
\rho_{S, \uparrow} & :=\operatorname{argmax}_{\rho_{S}} \operatorname{Tr}\left[\rho_{S}\left(A_{S}^{\prime}-A_{S}\right)\right], \\
\rho_{S, \downarrow} & :=\operatorname{argmin}_{\rho_{S}} \operatorname{Tr}\left[\rho_{S}\left(A_{S}^{\prime}-A_{S}\right)\right],
\end{aligned}
$$

where we used the abbreviation $A_{S}^{\prime}=U_{S}^{\dagger} A_{S} U_{S}$. Using the definition (37), $\mathcal{A}_{U_{S}}$ is expressed as

$$
\mathcal{A}_{U_{S}}=\frac{\left\langle A_{S}^{\prime}-A_{S}\right\rangle_{\rho_{S, \uparrow}}+\left\langle A_{S}^{\prime}-A_{S}\right\rangle_{\rho_{S, \downarrow}}}{2} .
$$

We write the final state of $E$ in actual dynamics with the initial state $\rho_{S, i}(i=\uparrow, \downarrow, \uparrow+\downarrow)$ as

$$
\sigma_{E, i}:=\operatorname{Tr}_{S}\left[U_{S E}\left(\rho_{S, i} \otimes \rho_{E}\right) U_{S E}^{\dagger}\right]
$$

We now state the aforementioned three relations (a)-(c) in a concrete form. First, the relation (a) is represented by the following inequality:

$$
L\left(\sigma_{E, \uparrow}, \sigma_{E, \downarrow}\right) \leqslant 2 \sqrt{2} \delta\left(\rho_{S, \uparrow+\downarrow}\right) .
$$

For $\delta \leqslant 1 / 2 \sqrt{2}$, a stronger inequality,

$$
L\left(\sigma_{E, \uparrow}, \sigma_{E, \downarrow}\right) \leqslant 2 \delta\left(\rho_{S, \uparrow+\downarrow}\right),
$$

is satisfied. These inequalities indicate a clear connection between the distance of two final states in $E$ and the accuracy of implementation (for the initial state $\rho_{S, \uparrow+\downarrow}$ ). We note that these inequalities apply even if $U_{S E}$ does not commute with $A_{S}+A_{E}$, and even if the dynamics of $S E$ is not unitary. We prove the generalized version of these inequalities in Appendix A.

Next, the relation (b) is represented by the following inequality:

$$
V_{\sigma_{E, \uparrow}}\left(A_{E}\right)+V_{\sigma_{E, \downarrow}}\left(A_{E}\right) \leqslant 2\left(V_{\rho_{E}}\left(A_{E}\right)+\Delta_{A_{S}}\right) .
$$

The term $\Delta_{A_{S}}$ is a correction term. This inequality connects the variance in the final state and that in the initial state.

Finally, the relation (c) is represented by the following inequality:

$$
2 \mathcal{A}_{U_{S}}-4 \delta\left(\rho_{S, \uparrow+\downarrow}\right) \Delta_{A_{S}} \leqslant \Delta \leqslant 2 \Delta_{A_{S}}
$$

where we set $\Delta:=\left|\operatorname{Tr}\left[\left(\sigma_{E, \uparrow}-\sigma_{E, \downarrow}\right) A_{E}\right]\right|$. Again $\delta\left(\rho_{S, \uparrow+\downarrow}\right) \Delta_{A_{S}}$ is a correction term. This inequality connects the degree of violation of the conservation of $A$ and the difference between the expected change in $A_{S}$ with the initial state $\rho_{S, \uparrow}$ and $\rho_{S, \downarrow}$. We prove these two inequalities in Appendix B. 
Proof. Theorem 1 We initially note that $\delta_{\mathcal{I}} \geqslant \delta\left(\rho_{S, \uparrow+\downarrow}\right)$. Therefore, to prove (36), we only have to show the inequality

$$
\sqrt{\mathcal{F}_{\rho_{E}}\left(A_{E}\right)} \geqslant \frac{\mathcal{A}_{U_{S}}}{\delta\left(\rho_{S, \uparrow+\downarrow}\right)}-6 \Delta_{A_{S}}
$$

for any implementation set $\left(\mathcal{H}_{E}, A_{E}, \rho_{E}, U_{S E}\right)$ satisfying $\delta_{\mathcal{I}} \leqslant$ $\delta$ for the desired unitary $U_{S}$. We divide the problem into two cases: $\delta>\mathcal{A}_{U_{S}} / 6 \Delta_{A_{S}}$ and $\delta \leqslant \mathcal{A}_{U_{S}} / 6 \Delta_{A_{S}}$. The former is trivial because in this case the right-hand side of (44) is negative while the quantum Fisher information $\mathcal{F}$ is always non-negative. Therefore, if we prove (44) for the case of $0<\delta \leqslant \mathcal{A}_{U_{S}} / 6 \Delta_{A_{S}}$, the inequality (44) holds for arbitrary $0<\delta \leqslant \sqrt{2}$. In the following, we consider the latter case: $\delta \leqslant \mathcal{A}_{U_{S}} / 6 \Delta_{A_{S}}$.

We first show (44) in the case that $\rho_{E}$ is a pure state. Since $\delta \leqslant \mathcal{A}_{U_{S}} / 6 \Delta_{A_{S}} \leqslant 1 / 6$, the stronger inequality (41) is satisfied, that suggests

$$
L\left(\sigma_{E, \uparrow}, \sigma_{E, \downarrow}\right) \leqslant 2 \delta\left(\rho_{S, \uparrow+\downarrow}\right) .
$$

Substituting the above relation, (42), and (43), into Lemma 1,

$$
\Delta \leqslant L\left(\sigma_{E, \uparrow}, \sigma_{E, \downarrow}\right)\left(V_{\sigma_{E, \uparrow}}\left(A_{E}\right)+V_{\sigma_{E, \downarrow}}\left(A_{E}\right)+\Delta\right)
$$

and, using $\Delta \leqslant 2 \Delta_{A_{S}}$, we obtain

$$
\mathcal{A}_{U_{S}} \leqslant \delta\left(\rho_{S, \uparrow+\downarrow}\right)\left[2 V_{\rho_{E}}\left(A_{E}\right)+6 \Delta_{A_{S}}\right] .
$$

Note that (47) holds for all $0<\delta\left(\rho_{S, \uparrow+\downarrow}\right) \leqslant \sqrt{2}$, since (47) is trivial in the case of $\mathcal{A}_{U_{S}} / 6 \Delta_{A_{S}}<\delta\left(\rho_{S, \uparrow+\downarrow}\right)$. By definition of the quantum Fisher information (7), $2 V_{\rho_{E}}\left(A_{E}\right)=\sqrt{\mathcal{F}_{\rho_{E}}\left(A_{E}\right)}$ holds for a pure state $\rho$. Thus, we obtain (44) for the case that $\rho_{E}$ is pure.

Next, we show (44) in the case that $\rho_{E}$ is a mixed state. We expand the initial state of the external system as $\rho_{E}:=$ $\sum_{j} p_{j} \phi_{E, j}$ satisfying $\mathcal{F}_{\rho_{E}}\left(A_{E}\right)=4 \sum_{j} p_{j} V_{\phi_{E, j}}^{2}\left(A_{E}\right)$. We denote $\delta\left(\rho_{S, \uparrow+\downarrow}\right)$ for the case that the initial state of $E$ is $\phi_{E, j}$ by $\delta_{j}$ :

$$
\delta_{j}:=L_{e}\left(\rho_{S, \uparrow+\downarrow}, \Lambda_{U_{S}^{\dagger}} \circ \Lambda_{S, j}\right),
$$

where $\Lambda_{S, j}$ is the dynamics of $S$ for the case that $\phi_{j}$ is the initial state of $E$, i.e., $\Lambda_{S, j}(\ldots):=\operatorname{Tr}_{E}\left[U_{S E}\left(\ldots \otimes \phi_{E, j}\right) U_{S E}^{\dagger}\right]$. The inequality (44) for pure states, which we earlier proved, yields

$$
2 V_{\phi_{E, j}}\left(A_{E}\right) \geqslant \frac{\mathcal{A}_{U_{S}}}{\delta_{j}}-6 \Delta_{A_{S}}
$$

for any $j$. By defining $k(x):=\left(\max \left\{0, \frac{\mathcal{A}_{U_{S}}}{x}-6 \Delta_{A_{S}}\right\}\right)^{2},(49)$ and the downward convexity of $k(x)$ yield ${ }^{x}$

$\mathcal{F}_{\rho_{E}}\left(A_{E}\right)=4 \sum_{j} p_{j} V_{\phi_{E, j}}\left(A_{E}\right)^{2} \geqslant \sum_{j} p_{j} k\left(\delta_{j}\right) \geqslant k\left(\sum_{j} p_{j} \delta_{j}\right)$.

Hence, to prove (44) for a mixed state, it suffices to show

$$
\sum_{j} p_{j} \delta_{j} \leqslant \delta\left(\rho_{S, \uparrow+\downarrow}\right),
$$

because the function $k$ is nonincreasing.

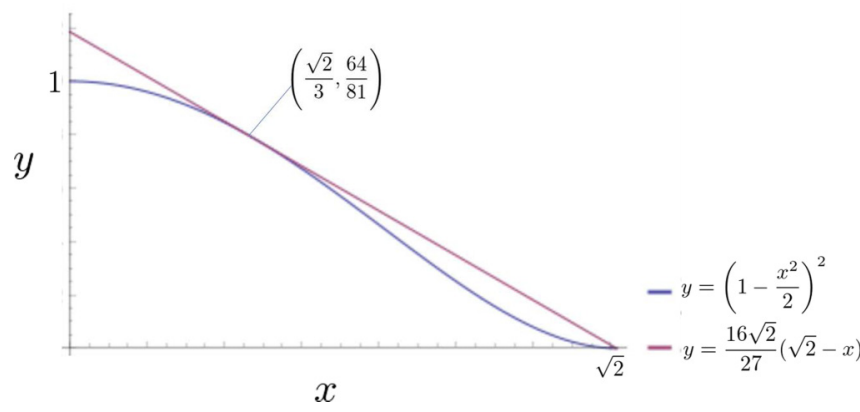

FIG. 6. Graphs of the functions $y=g(x)=\left(1-x^{2} / 2\right)^{2}$ and $16 \sqrt{2} / 27(\sqrt{2}-x)$. The function $y=l(x):=16 \sqrt{2} / 27(\sqrt{2}-x)$ is the tangent line on $y=g(x)$ at the point $(x, y)=(\sqrt{2} / 3,64 / 81)$. Since $g(x)$ is upward convex for $0 \leqslant x \leqslant \sqrt{2} / 3$, the function $g^{\prime}$ defined in (53) is upward convex and satisfies $g \leqslant g^{\prime}$.

Finally, we shall show (51). We employ the following equality:

$$
\begin{aligned}
(1 & \left.-\frac{\delta\left(\rho_{S, \uparrow+\downarrow}\right)^{2}}{2}\right)^{2} \\
& =\left\langle\psi_{S R, \uparrow+\downarrow}\left|U_{S}^{\dagger} \Lambda_{S}\left(\psi_{S R, \uparrow+\downarrow}\right) U_{S}\right| \psi_{S R, \uparrow+\downarrow}\right\rangle \\
& =\sum_{j} p_{j}\left\langle\psi_{S R, \uparrow+\downarrow}\left|U_{S}^{\dagger} \Lambda_{S, j}\left(\psi_{S R, \uparrow+\downarrow}\right) U_{S}\right| \psi_{S R, \uparrow+\downarrow}\right\rangle \\
& =\sum_{j} p_{j}\left(1-\frac{\delta_{j}^{2}}{2}\right)^{2}
\end{aligned}
$$

where $\psi_{S R, \uparrow+\downarrow}$ is the purification of $\rho_{S, \uparrow+\downarrow}$.

To show (51) from (52), we define $g(x):=\left(1-x^{2} / 2\right)^{2}$. As we show in Fig. 6, the function $y=l(x):=16 \sqrt{2} / 27(\sqrt{2}-$ $x)$ is the tangent line on at the point $(x, y)=(\sqrt{2} / 3,64 / 81)$. Since $g(x)$ is upward convex for $0 \leqslant x \leqslant \sqrt{2} / 3$, the following function $g^{\prime}$ is upward convex and satisfies $g \leqslant g^{\prime}$ :

$$
g^{\prime}(x):= \begin{cases}g(x) & \left(0 \leqslant x \leqslant \frac{\sqrt{2}}{3}\right) \\ l(x) & \left(\frac{\sqrt{2}}{3}<x \leqslant \sqrt{2}\right) .\end{cases}
$$

Therefore, by using Jensen's inequality, we obtain the following inequality for any probability distribution $\left\{q_{j}\right\}$ and real numbers $0 \leqslant x_{j} \leqslant \sqrt{2}$ :

$$
\sum_{j} q_{j} g\left(x_{j}\right) \leqslant \sum_{j} q_{j} g^{\prime}\left(x_{j}\right) \leqslant g^{\prime}\left(\sum_{j} q_{j} x_{j}\right) .
$$

As we have pointed out at the beginning of this proof, we only have to show (44) for the case of $\delta \leqslant \frac{\mathcal{A}_{U_{S}}}{6 \Delta_{A_{S}}}$. Due to $\mathcal{A}_{U_{S}} \leqslant \Delta_{A_{S}}$, we have $\frac{\mathcal{A}_{U_{S}}}{6 \Delta_{A_{S}}} \leqslant 1 / 6$. Therefore, from (53) and $\delta\left(\rho_{S, \uparrow+\downarrow}\right) \leqslant$ $\delta \leqslant 1 / 6$, we obtain

$$
g^{\prime}\left(\delta\left(\rho_{S, \uparrow+\downarrow}\right)\right)=g\left[\delta\left(\rho_{S, \uparrow+\downarrow}\right)\right] .
$$

Due to (52), we obtain

$$
g\left[\delta\left(\rho_{S, \uparrow+\downarrow}\right)\right]=\sum_{j} p_{j} g\left(\delta_{j}\right) .
$$


Combining (54)-(56), we obtain

$$
g^{\prime}\left[\delta\left(\rho_{S, \uparrow+\downarrow}\right)\right] \leqslant g^{\prime}\left(\sum_{j} p_{j} \delta_{j}\right) .
$$

Since $g^{\prime}$ is a nonincreasing function of $x$, we obtain (51).

\section{The proof of (22) for pure states}

We consider the inequality for a single initial state (22). Because the complete proof is a little complicated, we here only prove it for pure states $\rho_{S}$ and $\rho_{E}$ using several inequalities that are shown in Appendix. We shall present a complete proof in Appendix D.

We first introduce some symbols used in the proof. We denote the desired final state and realized final state by

$$
\begin{gathered}
\left|\rho_{S}^{\prime}\right\rangle:=U_{S}\left|\rho_{S}\right\rangle, \\
\left|\Psi_{S E}^{\prime}\right\rangle:=U_{S E}\left|\rho_{S}\right\rangle \otimes\left|\rho_{E}\right\rangle, \\
\sigma_{E}:=\operatorname{Tr}_{S}\left[\Psi_{S E}^{\prime}\right], \\
\sigma_{S}:=\operatorname{Tr}_{E}\left[\Psi_{S E}^{\prime}\right] .
\end{gathered}
$$

In a similar manner, the final state corresponding to the initial state $\psi_{i}$ is denoted by

$$
\begin{gathered}
\left|\psi_{i}^{\prime}\right\rangle:=U_{S}\left|\psi_{i}\right\rangle, \\
\left|\Psi_{i, S E}^{\prime}\right\rangle:=U_{S E}\left|\psi_{i}\right\rangle \otimes\left|\rho_{E}\right\rangle, \\
\sigma_{i, E}:=\operatorname{Tr}_{S}\left[\Psi_{i, S E}^{\prime}\right], \\
\sigma_{i, S}:=\operatorname{Tr}_{E}\left[\Psi_{i, S E}^{\prime}\right] .
\end{gathered}
$$

Note that now $\rho_{S}, \rho_{S}^{\prime} \psi_{i}$, and $\psi_{i}^{\prime}$ are pure and that

$$
\begin{aligned}
& 1-\frac{\delta\left(\rho_{S}\right)^{2}}{2}=F\left(\sigma_{S}, \rho_{S}^{\prime}\right), \\
& 1-\frac{\delta\left(\psi_{i}\right)^{2}}{2}=F\left(\sigma_{i, S}, \psi_{i}^{\prime}\right)
\end{aligned}
$$

hold. Therefore, due to Uhlmann's theorem [43], there are two pure states $\phi_{E}^{\prime}$ and $\phi_{i, E}^{\prime}$ satisfying

$$
\begin{array}{r}
\left\langle\Psi_{S E}^{\prime} \mid \rho_{S}^{\prime} \otimes \phi_{E}^{\prime}\right\rangle=F\left(\sigma_{S}, \rho_{S}^{\prime}\right)=1-\frac{\delta\left(\rho_{S}\right)^{2}}{2}, \\
\left|\left\langle\Psi_{i, S E}^{\prime} \mid \psi_{i}^{\prime} \otimes \phi_{i, E}^{\prime}\right\rangle\right|=F\left(\sigma_{i, S}, \psi_{i}^{\prime}\right)=1-\frac{\delta\left(\psi_{i}\right)^{2}}{2},
\end{array}
$$

where we wrote $|\rho\rangle \otimes|\phi\rangle$ as $|\rho \otimes \phi\rangle$. We also employ the abbreviation

$$
\bar{\delta}^{2}\left(\rho_{S},\left\{\psi_{i}\right\}\right):=\delta\left(\rho_{S}\right)^{2}+\sum_{i} r_{i} \delta\left(\psi_{i}\right)^{2} .
$$

Since (22) becomes trivial if $\bar{\delta}>\frac{\chi\left(\rho_{s}\left\{\psi_{i}\right\}\right)}{20 \Delta_{A_{S}}}$, we hereafter focus on the case of $\bar{\delta} \leqslant \frac{\chi\left(\rho_{s},\left\{\psi_{i}\right\}\right)}{20 \Delta_{A_{S}}}$.

Similar to (36), the derivation of (22) is based on the relations corresponding to Lemma 1 and the relations (a)-(c). Let us start from the relation (c). The relation (c) in this case is represented as

$$
\chi\left(\rho_{S},\left\{\psi_{i}\right\}\right) \leqslant \sqrt{\sum_{i} r_{i} \Delta_{i}^{2}}+\Delta^{\prime}+4 \bar{\delta}\left(\rho_{S}\right) \Delta_{A_{S}},
$$

where we defined

$$
\begin{aligned}
\Delta_{i} & :=\left|\left\langle A_{E}\right\rangle_{\sigma_{i, E}}-\left\langle A_{E}\right\rangle_{\phi_{E}^{\prime}}\right|, \\
\Delta^{\prime} & :=\left|\left\langle A_{E}\right\rangle_{\sigma_{E}}-\left\langle A_{E}\right\rangle_{\phi_{E}^{\prime}}\right| .
\end{aligned}
$$

To bound $\Delta_{i}$ and $\Delta^{\prime}$, we use Lemma 1. Using Lemma 1, (68), and $\delta\left(\rho_{S}\right) \leqslant \tilde{\delta}<1$, the quantity $\Delta^{\prime}$ is bounded as follows:

$$
\Delta^{\prime} \leqslant \frac{\delta\left(\rho_{S}\right)}{1-\delta\left(\rho_{S}\right)}\left[V_{\sigma_{E}}\left(A_{E}\right)+V_{\phi_{E}^{\prime}}\left(A_{E}\right)\right] .
$$

We also bound $\sum_{i} r_{i} \Delta_{i}^{2}$ by using Lemma 1 in the following form:

$$
\sum_{i} r_{i} \Delta_{i}^{2} \leqslant \sum_{i} r_{i} L\left(\sigma_{i, E}, \phi_{E}^{\prime}\right)^{2}\left[V_{\sigma_{i, E}}\left(A_{E}\right)+V_{\phi_{E}^{\prime}}\left(A_{E}\right)+\Delta_{i}\right]^{2} .
$$

The third term $\Delta_{i}$ in the right-hand side of (74) is bounded by $\Delta^{\prime}$ and $\Delta_{A_{S}}$ as follows (its proof is in Appendix D):

$$
\Delta_{i} \leqslant \Delta^{\prime}+2 \Delta_{A_{S}} .
$$

We note that $V_{\rho_{E}}\left(A_{E}\right)$ has a direct connection to the Fisher information $\mathcal{F}_{\rho_{E}}\left(A_{E}\right)$ in the case with a pure state. Therefore, we obtain (22) by bounding the three remaining terms $\sum_{i} r_{i} L\left(\sigma_{i, E}, \phi_{E}^{\prime}\right)^{2}, V_{\sigma_{i, E}}\left(A_{E}\right)$, and $V_{\phi_{E}^{\prime}}\left(A_{E}\right)$ by $V_{\rho_{E}}\left(A_{E}\right)$ and $\bar{\delta}\left(\rho_{S},\left\{\psi_{i}\right\}\right)$. To do this, we use the relations (a)-(c).

The relation (a) in this case is represented as

$$
\sum_{i} r_{i} L\left(\phi_{E}^{\prime}, \sigma_{i, E}\right)^{2} \leqslant 4 \bar{\delta}\left(\rho_{S},\left\{\psi_{i}\right\}\right)^{2},
$$

that is close to the relation (40). The version relation (b) in this case is represented as

$$
\begin{gathered}
V_{\sigma_{i, E}}\left(A_{E}\right) \leqslant V_{\rho_{E}}\left(A_{E}\right)+\Delta_{A_{S}}, \\
V_{\phi_{E}^{\prime}}\left(A_{E}\right) \leqslant \frac{\sqrt{\frac{1}{4} \Delta_{A_{S}}^{2}+V_{\rho_{E}}\left(A_{E}\right)^{2}}}{1-\frac{\delta^{2}\left(\rho_{S}\right)}{2}}
\end{gathered}
$$

that are close to the relation (42). These relations are shown in a similar manner to those for (40) and (42), and shown in Appendix D. Combining these relations and evaluating all correction terms, we arrive at the desired relation (22).

\section{DERIVATION OF THE SUFFICIENT CONDITION OF COHERENCE COST (THEOREM 2)}

We prove Theorem 2 by using the following lemma:

Lemma 2. For $\zeta \geqslant 9 \mathcal{A}_{U_{S}} / 2 \sqrt{2}$, there exists an implementation set $\mathcal{I}_{\zeta}=\left(\mathcal{H}_{E}, A_{E}, \phi_{\zeta}, U_{S E}\right)$ the $\mathcal{H}_{E}$ of which is a finite-dimensional system, and $\mathcal{I}_{\zeta}$ provides a good implementation of $U_{S}$ in the following sense:

$$
\begin{gathered}
\delta_{\mathcal{I}_{\zeta}} \leqslant \frac{\mathcal{A}_{U_{S}}}{2 \zeta}\left(1+\frac{\Delta_{A_{S}}}{\sqrt{2} \zeta}\right), \\
\mathcal{F}_{\phi_{\zeta}}\left(A_{E}\right)=4 \xi^{2} .
\end{gathered}
$$

We leave the proof of Lemma 2 to Appendix $C$. 
Proof. Theorem 2 Given an arbitrary $U_{S}$ on $S$, arbitrary precision $\delta$ with $0 \leqslant \delta \leqslant \frac{4 \sqrt{2} \mathcal{A}_{U_{S}}}{9 \Delta_{A_{S}}}$, and an arbitrary real number $\mathcal{F}$ satisfying $\sqrt{\mathcal{F}} \geqslant \frac{\mathcal{A}_{U_{S}}}{\delta}+\sqrt{2} \Delta_{A_{S}}$, we construct an implementation set $\mathcal{I}$ for $U_{S}$ satisfying $\delta_{\mathcal{I}} \leqslant \delta$ and $\mathcal{F}_{\rho_{E}}\left(A_{E}\right)=$ $\mathcal{F}$. We set a real positive number $\zeta$ as follows:

$$
2 \zeta:=\sqrt{\mathcal{F}}
$$

Here, we show that the implementation set $\mathcal{I}_{\zeta}=$ $\left(\mathcal{H}_{E}, A_{E}, \phi_{\zeta}, U_{S E}\right)$ constructed in Lemma 2 is $\mathcal{I}$ which we seek. Namely, we show $\mathcal{F}_{\phi_{\zeta}}\left(A_{E}\right)=\mathcal{F}$ and $\delta_{\mathcal{I}_{\zeta}} \leqslant \delta$. The relation $\mathcal{F}_{\phi_{\zeta}}\left(A_{E}\right)=\mathcal{F}$ is easily derived from (80) and (81). To show $\delta_{\mathcal{I}_{\zeta}} \leqslant \delta$, let us substitute (81) into (79):

$$
\delta_{\mathcal{I}_{\zeta}} \leqslant \frac{\mathcal{A}_{U_{S}}}{\sqrt{\mathcal{F}}}\left(1+\frac{\sqrt{2} \Delta_{A_{S}}}{\sqrt{\mathcal{F}}}\right) .
$$

By substituting $\sqrt{\mathcal{F}} \geqslant \frac{\mathcal{A}_{U_{S}}}{\delta}+\sqrt{2} \Delta_{A_{S}}$ into the above, we obtain

$$
\begin{aligned}
\delta_{\mathcal{I}_{\zeta}} & \leqslant \delta \frac{\mathcal{A}_{U_{S}}}{\mathcal{A}_{U_{S}}+\sqrt{2} \delta \Delta_{A_{S}}}\left(1+\frac{\sqrt{2} \delta \Delta_{A_{S}}}{\mathcal{A}_{U_{S}}+\sqrt{2} \delta \Delta_{A_{S}}}\right) \\
& =\delta \frac{\left(\mathcal{A}_{U_{S}}^{2}+2 \sqrt{2} \mathcal{A}_{U_{S}} \delta \Delta_{A_{S}}\right)}{\left(\mathcal{A}_{U_{S}}+\sqrt{2} \delta \Delta_{A_{S}}\right)^{2}} \\
& \leqslant \delta
\end{aligned}
$$

which means that $\mathcal{I}_{\zeta}$ implements $U_{S}$ within error $\delta$.

\section{SUMMARY AND DISCUSSION}

In this paper, we established simple relations between quantum coherence and asymmetry (violation of a conservation law). The coherence cost to realize unitary dynamics in a partial system under a symmetry (a conservation law) in a total system is asymptotically equal to the ratio between the degree of asymmetry of the implemented unitary and the implementation error. We derive the upper and lower bounds for the coherence cost that are asymptotically identical in the region where the error is small. This asymptotic equation quantitatively links two fundamental concepts in physics, i.e., symmetry and coherence.

Our results are applicable even when the whole system has multiple conservation laws. If the desired unitary dynamics alters two physical quantities and if the two physical quantities are conserved in the total system, then the external system must have the coherence required by Theorem 1 for each quantity.

Since our results are valid for any unitary operation, there are various applications of our results. In this paper, applications to the implementation of quantum heat engines, resource theories, and entanglement erasure are described as examples. In addition, our results are applicable to implementation of a time-dependent Hamiltonian and some controls while maintaining the quantum superposition.

The relation between our results and previous tradeoff relations in implementation of unitary dynamics [12-16] is as follows. The results given by Ozawa showed a tradeoff relation between accuracy of implementation of the CNOT gate and fluctuation of a conserved quantity [12]. Although these re- sults were generalized to other various unitary gates [13-15], it was an open problem whether there is a similar tradeoff relation for general unitary gates. In Ref. [16], this open problem was solved. Reference [16] also showed that the fluctuation must have quantum origin. However, the bound given by Ref. [16] was not tight. In this paper, we improve the bound in Ref. [16]. We show the asymptotic optimality of the improved bound, and give an asymptotic equality of coherence cost.

The given asymptotic equality for the coherence cost also has a close relation to the Wigner-Araki-Yanase theorem [70-74], that is a very famous theorem for quantum measurements. Particularly, an asymptotic equality of the coherence cost for quantum measurements under conservation laws was recently given [74]. The equality given in Ref. [74] has the same form as (21).

Is there a similar tradeoff equality for arbitrary CPTP maps? Here, we present a possible extension of our result. Let us consider an arbitrary CPTP map $\mathcal{E}_{S}$ on $S$. We will implement this CPTP map by using the same type of implementation set $\mathcal{I}=\left(\mathcal{H}_{E}, A_{E}, \rho_{E}, U_{S E}\right)$. Its total dynamics $U_{S E}$ conserves $A_{S}+A_{E}$ and the initial state $\rho_{E}$ might have coherence, i.e., $\left[U_{S E}, A_{S}+A_{E}\right]$ must be zero and $\mathcal{F}_{\rho_{E}}\left(A_{E}\right)$ can be zero. To define the degree of asymmetry (violation of the conservation of $A_{S}$ ), we consider another type of implementation $\mathcal{J}=\left(\mathcal{H}_{E}, A_{E}, \eta_{E}, V_{S E}\right)$, the initial state of which does not have coherence, that is, $\mathcal{F}_{\rho_{E}}\left(A_{E}\right)$ must be zero, and $V_{S E}$ might not conserve $A_{S}+A_{E}$, that is, $\left[V_{S E}, A_{S}+A_{E}\right]$ might be nonzero. We define the degree of asymmetry of $\mathcal{E}_{S}$ as the minimum degree of asymmetry in all possible $\mathcal{J}$ that implement $\mathcal{E}_{S}$ with no error [75]:

$$
\mathcal{A}_{\mathcal{E}_{S}}:=\min _{\mathcal{J}: \delta_{\mathcal{J}}=0} \mathcal{A}_{V_{S E}} .
$$

We also define the coherence cost of $\mathcal{E}_{S}$ as

$$
\mathcal{F}_{\delta}^{\mathrm{cost}}\left[\mathcal{E}_{S}\right]:=\min _{\mathcal{I} \models_{\delta} \mathcal{E}_{S}} \mathcal{F}_{\rho_{E}}\left(A_{E}\right)
$$

Note that if $\mathcal{E}_{S}=U_{S}$, the quantities $\mathcal{A}_{\Lambda_{S}}$ and $\mathcal{F}_{\delta}^{\text {cost }}\left[\mathcal{E}_{S}\right]$ reduce to $\mathcal{A}_{U_{S}}$ and $\mathcal{F}_{\delta}^{\text {cost }}\left[U_{S}\right]$, respectively. Hence, $\mathcal{A}_{\Lambda_{S}}$ and $\mathcal{F}_{\delta}^{\text {cost }}\left[\Lambda_{S}\right]$ are generalizations of $\mathcal{A}_{U_{S}}$ and $\mathcal{F}_{\delta}^{\text {cost }}\left[U_{S}\right]$. Theorem 2 provides the same form of inequality with these quantities:

$$
\sqrt{\mathcal{F}_{\delta}^{\text {cost }}\left[\mathcal{E}_{S}\right]} \leqslant \frac{\mathcal{A}_{\mathcal{E}_{S}}}{\delta}+\sqrt{2} \Delta_{A_{S}} .
$$

However, unfortunately we do not have an inequality similar to Theorem 1. If such an inequality is shown, we obtain the following asymptotic relation in a concise form:

$$
\text { Conjecture: } \sqrt{\mathcal{F}_{\delta}^{\text {cost }}\left[\mathcal{E}_{S}\right]}=\frac{\mathcal{A}_{\mathcal{E}_{S}}}{\delta}+O\left(\Delta_{A_{S}}\right) .
$$

We leave this problem as a future work.

\section{ACKNOWLEDGMENTS}

We thank Hiroshi Nagaoka, Tomohiro Ogawa, Satoshi Ishizaka, Eyuri Wakakuwa, and Yui Kuramochi for the fruitful discussion and helpful comments. The present work was supported by Japan Society for the Promotion of Science Grants-in-Aid for Scientific Research No. JP19K14610 
(H.T.), No. JP19K14615 (N.S.), No. JP25103003 (K.S.), and No. JP16H02211 (K.S.).

APPENDIX A: PROOF OF (40) AND (41)—NO-CORRELATION LEMMA

In this Appendix, we prove (40) and (41). More precisely, we prove the following generalized version of (40) and (41). This lemma is an improved version of Eq. (16) in Ref. [16].

Lemma 3. Consider two quantum systems $A$ and $B$. Let $\Lambda_{A B}$ be a CPTP map on the composite system $A B$ and let $U_{A}$ be a unitary operation on $A$. We consider three possible initial states of $A: \rho_{A}^{(0)}, \rho_{A}^{(1)}$, and $\rho_{A}^{(0+1)}:=\left(\rho_{A}^{(0)}+\rho_{A}^{(1)}\right) / 2$. We write the initial state of $B$ as $\rho_{B}$. We refer to the final states of $A B$ and $B$ with the initial state $\rho_{A}^{(i)}(i=0,1,0+1)$ as

$$
\begin{gathered}
\sigma_{A B}^{(i)}:=\Lambda_{A B}\left(\rho_{A}^{(i)} \otimes \rho_{B}\right), \\
\sigma_{B}^{(i)}:=\operatorname{Tr}_{A}\left[\sigma_{A B}^{(i)}\right] .
\end{gathered}
$$

We refer to the time evolution of $A$ determined by $\Lambda_{A B}$ and $\rho_{B}$ as $\Lambda_{A}(\rho):=\operatorname{Tr}_{B}\left[\Lambda_{A B}\left(\rho \otimes \rho_{B}\right)\right]$. Using this symbol, we define the accuracy of implementation of $U_{A}$ by $\Lambda_{A B}$ for the initial states $\rho_{A}^{(i)}(i=0,1,0+1)$ as

$$
\delta_{U}^{(i)}:=L_{e}\left(\rho_{A}^{(i)}, \Lambda_{U_{A}^{\dagger}} \circ \Lambda_{A}\right) .
$$

In this setup, we have the following results.

(1) The following inequality holds:

$$
L\left(\sigma_{A B}^{(0)}, U_{A} \rho_{A}^{(0)} U_{A}^{\dagger} \otimes \sigma_{B}^{(0)}\right) \leqslant 2 \delta_{U}^{(0)} .
$$

(2) There exists a state $\sigma_{B}^{\prime(0+1)}$ of $B$ such that

$$
L\left(\sigma_{B}^{(0)}, \sigma_{B}^{\prime(0+1)}\right)+L\left(\sigma_{B}^{\prime(0+1)}, \sigma_{B}^{(1)}\right) \leqslant 2 \sqrt{2} \delta_{U}^{(0+1)} .
$$

Moreover, if $\delta_{U}^{(0+1)} \leqslant 1 / 2 \sqrt{2}$ holds, there exists a state $\sigma_{B}^{\prime(0+1)}$ of $B$ such that

$$
L\left(\sigma_{B}^{(0)}, \sigma_{B}^{\prime(0+1)}\right)+L\left(\sigma_{B}^{\prime(0+1)}, \sigma_{B}^{(1)}\right) \leqslant 2 \delta_{U}^{(0+1)} .
$$

If $\rho_{B}$ is a pure state and $\Lambda_{A B}$ is a unitary operation, the aforementioned $\sigma_{B}^{\prime(0+1)}$ is a pure state.

Proof. Lemma 3 We first introduce some symbols. We take the purification $\psi_{A R_{A}}^{(i)}$ of $\rho_{A}^{(i)}(i=0,1,0+1)$ such that $\rho_{R_{A}}^{(0)}:=$ $\operatorname{Tr}_{A}\left[\psi_{A R_{A}}^{(0)}\right]$ and $\rho_{R_{A}}^{(1)}:=\operatorname{Tr}_{A}\left[\psi_{A R_{A}}^{(1)}\right]$ are pure states and orthogonal to each other. In that case, $\left|\psi^{(0+1)}\right\rangle_{A R}=\left(\left|\psi^{(0)}\right\rangle_{A R}+\right.$ $\left.\left|\psi^{(1)}\right\rangle_{A R}\right) / \sqrt{2}$ holds. We write the purification of $\rho_{B}$ as $\psi_{B R_{B}}$. We employ the Steinspring representation [43] of $\Lambda_{A B}$, that is, we describe $\Lambda_{A B}(\rho)$ by using a pure state $\psi_{C}$ and a unitary transformation $U_{\mathrm{ABC}}$ as $\Lambda_{A B}(\rho)=\operatorname{Tr}_{C}\left[U_{\mathrm{ABC}}\left(\rho \otimes \psi_{C}\right) U_{\mathrm{ABC}}^{\dagger}\right]$. We denote the initial and final states of the total system $A R_{A} B R_{B} C$ by

$$
\begin{gathered}
\psi_{\text {tot }}^{(i)}:=\psi_{A R_{A}}^{(i)} \otimes \psi_{B R_{B}} \otimes \psi_{C}, \\
\psi_{\text {tot }}^{\prime(i)}:=U_{\mathrm{ABC}} \psi_{\mathrm{tot}}^{(i)} U_{\mathrm{ABC}}^{\dagger},
\end{gathered}
$$

respectively. We also denote the final states of $A R_{A}$ and $B R_{B} C$ by

$$
\begin{aligned}
\sigma_{A R_{A}}^{(i)} & :=\operatorname{Tr}_{B R_{B} C}\left[\psi_{\text {tot }}^{\prime(i)}\right], \\
\sigma_{B R_{B} C}^{(i)} & :=\operatorname{Tr}_{A R_{A}}\left[\psi_{\text {tot }}^{(i)}\right],
\end{aligned}
$$

respectively.
From Uhlmann's theorem, the definition of $\delta_{U}^{(i)}$,

$$
\delta_{U}^{(i)}=L\left(U_{A} \psi_{A R_{A}}^{(i)} U_{A}^{\dagger}, \sigma_{A R_{A}}^{(i)}\right),
$$

has another expression with a proper pure state $\phi_{B R_{B} C}^{\prime(i)}$ as

$$
\delta_{U}^{(i)}=L\left(U_{A} \psi_{A R_{A}}^{(i)} U_{A}^{\dagger} \otimes \phi_{B R_{B} C}^{\prime(i)}, \psi_{\text {tot }}^{\prime(i)}\right) .
$$

Owing to the contractivity of the Bures distance, by taking the partial trace of $A R_{A}$ in (A12) we obtain

$$
\delta_{U}^{(i)} \geqslant L\left(\phi_{B R_{B} C}^{\prime(i)}, \sigma_{B R_{B} C}^{(i)}\right) .
$$

We now derive (A5) and (A4) by using (A13). We first derive (A4). We start from the following triangle inequality:

$$
\begin{aligned}
& L\left(\psi_{\mathrm{tot}}^{\prime(i)}, U_{A} \psi_{A R_{A}}^{(i)} U_{A}^{\dagger} \otimes \sigma_{B R_{B} C}^{(i)}\right) \\
& \leqslant L\left(\psi_{\mathrm{tot}}^{\prime(i)}, U_{A} \psi_{A R_{A}}^{(i)} U_{A}^{\dagger} \otimes \phi_{B R_{B} C}^{\prime(i)}\right) \\
& \quad+L\left(U_{A} \psi_{A R_{A}}^{(i)} U_{A}^{\dagger} \otimes \phi_{B R_{B} C}^{(i)}, U_{A} \psi_{A R_{A}}^{(i)} U_{A}^{\dagger} \otimes \sigma_{B R_{B} C}^{(i)}\right) .
\end{aligned}
$$

The first and second terms of the right-hand side are bounded by (A12) and (A13), respectively, which yields

$$
L\left(\psi_{\text {tot }}^{\prime(i)}, U_{A} \psi_{A R_{A}}^{(i)} U_{A}^{\dagger} \otimes \sigma_{B R_{B} C}^{(i)}\right) \leqslant 2 \delta_{U}^{(i)} .
$$

By taking the partial trace of $A R_{A}$ in the above inequality, we obtain the desired relation (A4):

$$
L\left(\sigma_{A B}^{(i)}, U_{A} \rho_{A}^{(i)} U_{A}^{\dagger} \otimes \sigma_{B}^{(i)}\right) \leqslant 2 \delta_{U}^{(i)} .
$$

Next we show (A5). We note the following relation:

$$
\sigma_{B R_{B} C}^{(0+1)}=\frac{\sigma_{B R_{B} C}^{(0)}+\sigma_{B R_{B} C}^{(1)}}{2},
$$

which comes from a relation $\operatorname{Tr}_{A B R_{B} C}\left[\psi_{\text {tot }}^{\prime(a)}\right]=\rho_{R_{A}}^{(a)}$ for $a=$ 0,1 , and the fact that $\rho_{R_{A}}^{(0)}$ and $\rho_{R_{A}}^{(1)}$ are orthogonal to each other. Then, (A13) implies

$$
\delta_{U}^{(0+1)} \geqslant L\left(\phi_{B R_{B} C}^{\prime(0+1)}, \sigma_{B R_{B} C}^{(0+1)}\right)=L\left(\phi_{B R_{B} C}^{\prime(0+1)}, \frac{\sigma_{B R_{B} C}^{(0)}+\sigma_{B R_{B} C}^{(1)}}{2}\right),
$$

or, equivalently,

$$
F\left(\phi_{B R_{B} C}^{\prime(0+1)}, \frac{\sigma_{B R_{B} C}^{(0)}+\sigma_{B R_{B} C}^{(1)}}{2}\right) \geqslant 1-\frac{\left(\delta_{U}^{(0+1)}\right)^{2}}{2} .
$$

Noting that $\phi_{B R_{B} C}^{\prime(0+1)}$ is a pure state, the left-hand side of the above inequality is transformed into

$$
\begin{aligned}
& F\left(\phi_{B R_{B} C}^{(0+1)}, \frac{\sigma_{B R_{B} C}^{(0)}+\sigma_{B R_{B} C}^{(1)}}{2}\right)^{2} \\
& =\left\langle\phi_{B R_{B} C}^{(0+1)}\left|\frac{\sigma_{B R_{B} C}^{(0)}+\sigma_{B R_{B} C}^{(1)}}{2}\right| \phi_{B R_{B} C}^{\prime(0+1)}\right\rangle \\
& =\frac{1}{2} \sum_{i=0,1} F\left(\phi_{B R_{B} C}^{(0+1)}, \sigma_{B R_{B} C}^{(i)}\right)^{2} .
\end{aligned}
$$

Combining the above equations and the relation (1$\left.x^{2} / 2\right)^{2} \geqslant 1-x^{2}$, we obtain

$$
\frac{1}{2} \sum_{i=0,1} F\left(\phi_{B R_{B} C}^{(0+1)}, \sigma_{B R_{B} C}^{(i)}\right)^{2} \geqslant 1-\left(\delta_{U}^{(0+1)}\right)^{2},
$$


which can be evaluated as

$$
\begin{aligned}
& \left(\delta_{U}^{(0+1)}\right)^{2} \geqslant 1-\frac{1}{2} \sum_{i=0,1} F\left(\phi_{B R_{B} C}^{(0+1)}, \sigma_{B R_{B} C}^{(i)}\right)^{2} \\
& \geqslant 1-\frac{1}{2} \sum_{i=0,1} F\left(\phi_{B R_{B} C}^{(0+1)}, \sigma_{B R_{B} C}^{(i)}\right) \\
& =\frac{1}{4} \sum_{i=0,1} L\left(\phi_{B R_{B} C}^{(0+1)}, \sigma_{B R_{B} C}^{(i)}\right)^{2} \\
& \geqslant \frac{1}{8}\left(\sum_{i=0,1} L\left(\phi_{B R_{B} C}^{(0+1)}, \sigma_{B R_{B} C}^{(i)}\right)\right)^{2} \\
& \geqslant \frac{1}{8}\left(\sum_{i=0,1} L\left(\sigma_{B}^{(0+1)}, \sigma_{B}^{(i)}\right)\right)^{2} .
\end{aligned}
$$

This is equivalent to the desired relation (A5). Here, we defined $\sigma_{B}^{\prime(0+1)}:=\operatorname{Tr}_{R_{B} C}\left[\phi_{B R_{B} C}^{\prime(0+1)}\right]$, and we used the relation $X^{2}+Y^{2} \geqslant \frac{(X+Y)^{2}}{2}$ for positive numbers $X$ and $Y$ in the fourth line, and the contractivity of the Bures distance in the last line. We remark that if $\rho_{B}$ is pure and $\Lambda_{A B}$ is unitary, by following the above derivation without $R_{B} C$, we obtain the fact that $\sigma_{B}^{\prime(0+1)}$ is a pure state.

We finally derive (A6) when $\delta_{U}^{(0+1)} \leqslant 1 / 2 \sqrt{2}$ holds. Using again the fact that $\phi_{B R_{B} C}^{\prime(0+1)}$ is a pure state, (A19) reads

$$
\frac{1}{2} \sum_{i=0,1}\left(1-\frac{L\left(\phi_{B R_{B} C}^{(0+1)}, \sigma_{B R_{B} C}^{(i)}\right)^{2}}{2}\right)^{2} \geqslant\left(1-\frac{\left(\delta_{U}^{(0+1)}\right)^{2}}{2}\right)^{2} .
$$

Noting the relation

$$
\frac{1}{2}\left[\left(1-\frac{X^{2}}{2}\right)^{2}+\left(1-\frac{Y^{2}}{2}\right)^{2}\right] \leqslant\left(1-\frac{(X+Y)^{2}}{8}\right)^{2}
$$

for real numbers $X$ and $Y$ satisfying $0 \leqslant X+Y \leqslant 1$, we arrive at

$$
1-\frac{\left(\sum_{i=0,1} L\left(\phi_{B R_{B} C}^{(0+1)}, \sigma_{B R_{B} C}^{(i)}\right)\right)^{2}}{8} \geqslant 1-\frac{\left(\delta_{U}^{(0+1)}\right)^{2}}{2} .
$$

Here we used the relation $L\left(\phi_{B R_{B} C}^{\prime(0+1)}, \sigma_{B R_{B} C}^{(0)}\right)+$ $L\left(\phi_{B R_{B} C}^{\prime(0+1)}, \sigma_{B R_{B} C}^{(1)}\right) \leqslant 2 \sqrt{2} \delta_{U}^{(0+1)} \leqslant 1$, which follows from (A5), in application of (A24). By taking the partial trace of $R_{B} C$, the above inequality directly implies the desired relation (A6).

\section{APPENDIX B: PROOF OF (42) AND (43)}

Proof. (42) The conservation of $A_{S}+A_{E}$ under $U_{S E}$ yields

$$
\begin{gathered}
V_{\rho_{S, i}}^{2}\left(A_{S}\right)+V_{\rho_{E}}^{2}\left(A_{E}\right)=V_{\sigma_{S, i}}^{2}\left(A_{S}\right)+V_{\sigma_{E, i}}^{2}\left(A_{E}\right) \\
+2 \operatorname{Cov}_{A_{S}+A_{E}}\left[U_{S E}\left(\rho_{S, i} \otimes \rho_{E}\right) U_{S E}^{\dagger}\right],
\end{gathered}
$$

where $i \in\{\uparrow, \downarrow\}$ and $\sigma_{S, i}:=\operatorname{Tr}_{E}\left[U_{S E}\left(\rho_{S, i} \otimes \rho_{E}\right) U_{S E}^{\dagger}\right] . V_{\rho}\left(A_{S}\right)$ represents the standard deviation of the quantity $A_{S}$ in $\rho$, and $\operatorname{Cov}_{A_{S}+A_{E}}(\sigma)$ is the covariance between $A_{S}$ and $A_{E}$ with the state $\sigma$. Using a basic property of covariance $-V_{\sigma_{S, i}}\left(A_{S}\right) V_{\sigma_{E, i}}\left(A_{E}\right) \leqslant \operatorname{Cov}_{A_{S}+A_{E}}\left(U_{S E}\left(\rho_{S, i} \otimes \rho_{E}\right) U_{S E}^{\dagger}\right)$, we arrive at

$$
\begin{aligned}
& V_{\sigma_{E, i}}\left(A_{E}\right)-V_{\sigma_{S, i}}\left(A_{S}\right) \\
& \quad \leqslant \sqrt{V_{\sigma_{S, i}}^{2}\left(A_{S}\right)+V_{\sigma_{E, i}}^{2}\left(A_{E}\right)-2 V_{\sigma_{S, i}}\left(A_{S}\right) V_{\sigma_{E, i}}\left(A_{E}\right)} \\
& \quad \leqslant \sqrt{V_{\rho_{E}}^{2}\left(A_{E}\right)+V_{\rho_{S, i}}^{2}\left(A_{S}\right)} \\
& \quad \leqslant V_{\rho_{E}}\left(A_{E}\right)+V_{\rho_{S, i}}\left(A_{S}\right) .
\end{aligned}
$$

Using a relation $V_{\rho}\left(A_{S}\right) \leqslant \Delta_{A_{S}} / 2$ for any state $\rho$ and taking the sum of $i \in\{\uparrow, \downarrow\}$, we obtain (42).

Proof. (43) Let us introduce the following quantities:

$$
\begin{gathered}
\Delta_{i}:=\operatorname{Tr}\left[A_{E}\left(\sigma_{E, i}-\rho_{E}\right)\right]=\operatorname{Tr}\left[A_{S}\left(\rho_{S, i}-\sigma_{S, i}\right)\right], \\
\Delta_{U, i}:=\operatorname{Tr}\left[A_{S}\left(\rho_{S, i}-U_{S} \rho_{S, i} U_{S}^{\dagger}\right)\right],
\end{gathered}
$$

where $i \in\{\uparrow, \downarrow\}$ and $\sigma_{S, i}:=\operatorname{Tr}_{E}\left[U_{S E}\left(\rho_{S, i} \otimes \rho_{E}\right) U_{S E}^{\dagger}\right]$. The three quantities appearing in (43) can be written or evaluated in terms of the above quantities:

$$
\begin{aligned}
\Delta & =\left|\Delta_{\downarrow}-\Delta_{\uparrow}\right|, \\
\mathcal{A}_{U_{S}} & =\frac{\Delta_{U, \uparrow}-\Delta_{U, \downarrow}}{2}, \\
2 \delta\left(\rho_{S, i}\right) \Delta_{A_{S}} & \geqslant 2 L_{B}\left(\sigma_{S, i}, U_{S} \rho_{S, i} U_{S}^{\dagger}\right) \Delta_{A_{S}} \\
& \geqslant\left\|\sigma_{S, i}-U_{S} \rho_{S, i} U_{S}^{\dagger}\right\|_{1} \Delta_{A_{S}} \\
& \geqslant\left|\Delta_{i}-\Delta_{U, i}\right|,
\end{aligned}
$$

where $i \in\{\uparrow, \downarrow\},\|X\|_{1}:=\operatorname{Tr} \sqrt{X^{\dagger} X}$ is the trace norm, and we used $\|\rho-\sigma\|_{1} \leqslant 2 \sqrt{1-F^{2}(\rho, \sigma)} \leqslant 2 L_{B}(\rho, \sigma)[43]$ in (B7). Combining (B5)-(B7), we obtain

$$
\begin{aligned}
2 \mathcal{A}_{U_{S}} & =\left|\Delta_{U, \uparrow}-\Delta_{U, \downarrow}\right| \\
& \leqslant\left|\Delta_{\uparrow}-\Delta_{\downarrow}\right|+2\left[\delta\left(\rho_{S, \uparrow}\right)+\delta\left(\rho_{S, \downarrow}\right)\right] \Delta_{A_{S}} \\
& =\Delta+2\left[\delta\left(\rho_{S, \uparrow}\right)+\delta\left(\rho_{S, \downarrow}\right)\right] \Delta_{A_{S}} .
\end{aligned}
$$

Hence, proving

$$
\delta\left(\rho_{S, \uparrow}\right)+\delta\left(\rho_{S, \downarrow}\right) \leqslant 2 \delta\left(\rho_{S, \uparrow+\downarrow}\right)
$$

suffices to show the left-hand side inequality of the desired (43). We first define some symbols. We take purification $\psi_{S R_{S}, i}$ of $\rho_{S, i}(i=\uparrow, \downarrow, \uparrow+\downarrow)$ such that $\rho_{R_{S}, \uparrow}:=$ $\operatorname{Tr}_{S}\left[\psi_{S R_{S}, \uparrow}\right]$ and $\rho_{R_{S, \downarrow} \downarrow}:=\operatorname{Tr}_{S}\left[\psi_{S R_{S}, \downarrow}\right]$ are pure states and orthogonal to each other. In this case, $\left|\psi_{S R_{S}, \uparrow+\downarrow}\right\rangle=\left(\left|\psi_{S R_{S}, \uparrow}\right\rangle+\right.$ $\left.\left|\psi_{S R_{S}, \downarrow}\right\rangle\right) / \sqrt{2}$ holds. We denote the purification of $\rho_{E}$ by $\psi_{E R_{E}}$. We denote the initial and final states of the total system $S R_{S} E R_{E}$ by $\psi_{\mathrm{tot}, i}:=\psi_{S R_{S}, i} \otimes \psi_{E R_{E}}$ and $\psi_{\mathrm{tot}, i}^{\prime}:=$ $U_{S E} \psi_{\mathrm{tot}, i} U_{S E}^{\dagger}$, respectively. We also denote the final states of $S R_{S}$ and $E R_{E}$ by $\sigma_{S R_{S}, i}:=\operatorname{Tr}_{E R_{E}}\left[\psi_{\text {tot }, i}^{\prime}\right]$ and $\sigma_{E R_{E}, i}:=$ $\operatorname{Tr}_{S R_{S}}\left[\psi_{\text {tot, }, i}^{\prime}\right]$, respectively.

We recall the fact that $\delta(\rho)$ is expressed in terms of fidelity:

$$
1-\frac{\delta\left(\rho_{S, i}\right)^{2}}{2}=F\left(\sigma_{S R_{S}, i}, U_{S} \psi_{S R_{S}, i} U_{S}^{\dagger}\right) .
$$


Uhlmann's theorem implies that there is a proper pure state $\phi_{E R_{E}}^{\prime}$ on $E R_{E}$ such that

$$
\begin{aligned}
& F\left(\sigma_{S R_{S}, \uparrow+\downarrow}, U_{S} \psi_{S R_{S}, \uparrow+\downarrow} U_{S}^{\dagger}\right) \\
& \quad=\mid\left\langle\psi_{\mathrm{tot}, \uparrow+\downarrow}\left|U_{S}\right| \psi_{S R_{S}, \uparrow+\downarrow} \otimes \phi_{E R_{E}}^{\prime}\right\rangle .
\end{aligned}
$$

Using $\quad\left|\psi_{S R_{S}, \uparrow+\downarrow}\right\rangle=\left(\left|\psi_{S R_{S}, \uparrow}\right\rangle+\left|\psi_{S R_{S}, \downarrow}\right\rangle\right) / \sqrt{2}$ and $\left|\psi_{\text {tot }, \uparrow+\downarrow}\right\rangle=\left(\left|\psi_{\text {tot }, \uparrow}\right\rangle+\left|\psi_{\text {tot }, \downarrow}\right\rangle\right) / \sqrt{2}$, the right-hand side of the above relation is bounded from above as

$$
\begin{aligned}
& \mid\left\langle\psi_{\mathrm{tot}, \uparrow+\downarrow}\left|U_{S}\right| \psi_{S R_{S}, \uparrow+\downarrow} \otimes \phi_{E R_{E}}^{\prime}\right\rangle \\
& \quad=\left|\frac{1}{2} \sum_{m=\uparrow, \downarrow}\left\langle\psi_{\mathrm{tot}, m}\left|U_{S}\right| \psi_{S R_{S}, m} \otimes \phi_{E R_{E}}^{\prime}\right\rangle\right| \\
& \quad \leqslant \frac{1}{2} \sum_{m=\uparrow, \downarrow}\left|\left\langle\psi_{\mathrm{tot}, m}\left|U_{S}\right| \psi_{S R_{S}, m} \otimes \phi_{E R_{E}}^{\prime}\right\rangle\right| \\
& \quad \leqslant \frac{1}{2} \sum_{m=\uparrow, \downarrow} F\left(\sigma_{S R_{S}, m}, U_{S} \psi_{S R_{S}, m} U_{S}^{\dagger}\right) .
\end{aligned}
$$

Combining (B10)-(B12), we obtain (B9).

Next, let us show the right-hand side inequality of (43). It is easily shown as follows:

$$
\begin{aligned}
\Delta & \leqslant\left|\Delta_{\downarrow}\right|+\left|\Delta_{\uparrow}\right| \\
& =\left|\operatorname{Tr}\left[A_{S}\left(\rho_{S, \uparrow}-\sigma_{S, \uparrow}\right)\right]\right|+\left|\operatorname{Tr}\left[A_{S}\left(\rho_{S, \downarrow}-\rho_{S, \downarrow}^{\prime}\right)\right]\right| \\
& \leqslant 2 \Delta_{A_{S}} .
\end{aligned}
$$

Here we use (B3) in the second equality in (B13).

\section{APPENDIX C: PROOF OF LEMMA 2}

In this section, we prove Lemma 2 in the main text. In the proof, we use the following abbreviation for convenience:

$$
\Delta_{X}=\lambda_{\max }(X)-\lambda_{\min }(X),
$$

where $\lambda_{\max }(X)$ and $\lambda_{\min }(X)$ are the maximum and minimum eigenvalues of $X$. Note that $\mathcal{A}_{U_{S}}=\Delta_{A_{S}-U_{S}^{\dagger} A_{S} U_{S}} / 2$.

\section{Strategy of the proof: How to construct a "good" implementation set}

In Appendix C, we prove Lemma 2 implementation set $\mathcal{I}_{\zeta}$ which satisfies (79) and (80). In this subsection, we explain our strategy for how to construct such an implementation set.

To define an implementation set, we have to give an external system $E$, an initial state on $E$, and an interaction unitary dynamics $U_{S E}$ between $S$ and $E$. Our external system $E$ is divided into five subsystems: $S^{\prime}, E_{0}, E_{\alpha}, E_{\beta}$, and $E_{\gamma}$.

Let us explain the role of each subsystem. $S^{\prime}$ is an approximate copy system of $S$, the Hilbert space of which has the same dimension as $S$. The conserved quantity $A_{S^{\prime}}$ is very close to $A_{S}$, but all the eigenvalues of $A_{S^{\prime}}$ are integer multiples of a real positive number $1 / M^{2}$. As we will see later, $1 / M^{2}$ is equal to the minimum difference of eigenvalues of the conserved quantity $A$ in the main part of the external system. The subsystems $E_{\alpha}$ and $E_{\beta}$ are used to perform approximately a SWAP gate between $S$ and $S^{\prime}$. As we will show in the next subsection, when $M$ is very large, we can perform the SWAP gate with negligibly small coherence in $E_{\alpha}$ and $E_{\beta}$. The subsystem $E_{0}$ is

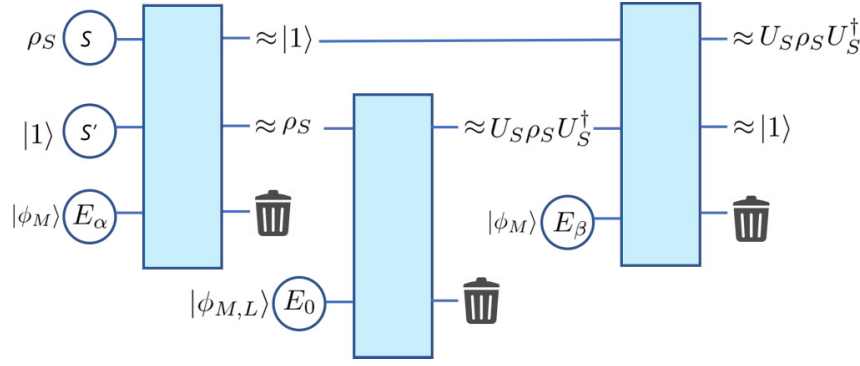

FIG. 7. Schematic of the sequence of use of the subsystems $S^{\prime}, \ldots, E_{\beta}$.

the main part of $E$, and is used to perform approximately $U_{S}$ on $S^{\prime}$. The $E_{0}$ is a discrete finite-dimensional ladder system, and contains an approximate Gaussian pure state, which will be defined in (C14). The order of use of $S^{\prime}, E_{0}, E_{\alpha}$, and $E_{\beta}$ is shown in Fig. 7.

As we will see later, with choosing parameters of $S^{\prime}$, $E_{0}, E_{\alpha}$, and $E_{\beta}$ properly, we can make the error $\delta_{\mathcal{I}_{\xi}}$ and the quantum Fisher information of $S^{\prime} E_{0} E_{\alpha} E_{\beta}$ smaller than $\frac{\mathcal{A}_{U_{S}}}{2 \zeta}\left(1+\frac{\Delta_{A_{S}}}{\sqrt{2 \zeta}}\right)$ and $4 \zeta^{2}$, respectively. To make the total Fisher information in $E$ equal to $4 \zeta^{2}$ and obtain (80), we use a two-dimensional system $E_{\gamma}$. Note that the two-dimensional system $E_{\gamma}$ does not interact with other systems. The role of $E_{\gamma}$ is only to make the total Fisher information in $E$ equal to $4 \zeta^{2}$.

\section{Preparation: Construction of a good implementation set}

In this subsection, we give a concrete definition of an implementation set $\mathcal{I}_{\zeta, L, M, \epsilon}=\left(\mathcal{H}_{E}^{L, M}, A_{E}^{L, M, \epsilon}, \phi_{\zeta}^{L, M}, U_{S E}^{L, M}\right)$ as a function of real positive numbers $\zeta$ and $\epsilon$ and positive integers $L$ and $M$, satisfying $M>L>8 d_{S} \max \left\{d_{S}\left\|A_{S}\right\|\right\}$ and $M / L \in$ $\mathcal{N}$ is a positive integer. As we will see later, $L$ determines the maximum and minimum eigenvalues of $A_{E_{0}}$, and $1 / M^{2}$ is equal to the minimum difference of eigenvalues of $A_{E_{0}}$. In the next subsection, we will construct $\mathcal{I}_{\zeta}$ in Lemma 2 from $\mathcal{I}_{\zeta, L, M, \epsilon}$. lows:

We define the Hilbert space of the external system as fol-

$$
\mathcal{H}_{E}^{L, M}:=\left(\otimes_{i=0}^{2 d_{S}+1} \mathcal{H}_{E_{i}}^{L, M}\right) \otimes \mathcal{H}_{S^{\prime}}^{M},
$$

where $d_{S}$ is the dimension of the Hilbert space of $S$, and the dimensions of $\mathcal{H}_{E_{i}}^{L, M}$ and $\mathcal{H}_{S_{i}}^{M}$ are

$$
\begin{aligned}
\operatorname{dim}\left(\mathcal{H}_{E_{i}}^{L, M}\right):= & \begin{cases}8 L M^{2} & (i=0) \\
2 M^{2}+1 & \left(1 \leqslant i \leqslant 2 d_{S}\right) \\
2 & \left(i=2 d_{S}+1\right),\end{cases} \\
& \operatorname{dim}\left(\mathcal{H}_{S^{\prime}}^{M}\right):=d_{S} .
\end{aligned}
$$

$S^{\prime}, E_{0}, \otimes_{k=1}^{d_{S}} E_{k}, \otimes_{k=1}^{d_{S}} E_{k+d_{S}}$, and $E_{2 d_{S}+1}$ correspond to $S^{\prime}, E_{0}$, $E_{\alpha}, E_{\beta}$, and $E_{\gamma}$ in subsection C 1 , respectively.

The conserved quantity $A_{E}^{L, M, \epsilon}$ is defined as follows:

$$
A_{E}^{L, M, \epsilon}=A_{E_{0}}^{L, M}+A_{E_{2 d_{S}+1}}^{\epsilon}+\sum_{i=1}^{2 d_{S}} A_{E_{i}}^{M}+\tilde{A}_{S^{\prime}}^{M},
$$


where

$$
\begin{gathered}
A_{E_{0}}^{L, M}:=\sum_{m=1}^{8 L M^{2}} x_{m}|m\rangle\left\langle\left. m\right|_{E_{0}},\right. \\
A_{E_{i}}^{L, M}:=A_{E_{d_{S}+i}}^{L, M}=\sum_{m=-M^{2}}^{M^{2}} \epsilon_{i} m|m\rangle\left\langle\left. m\right|_{E_{i}}\left(1 \leqslant i \leqslant d_{S}\right),\right. \\
A_{E_{2 d_{S}+1}^{L, M}}^{L}:=\epsilon|1\rangle\left\langle\left. 1\right|_{E_{2 d_{S}+1},}\right. \\
\tilde{A}_{S^{\prime}}^{M}:=\sum_{i=1}^{d_{S}} \tilde{h}_{i}|i\rangle\left\langle\left. i\right|_{S^{\prime}} .\right.
\end{gathered}
$$

Here, $x_{m}, \epsilon_{m}$, and $\tilde{h}_{i}$ are defined as

$$
\begin{gathered}
x_{m}:=-4 L+\frac{1}{M^{2}}\left(m-\frac{1}{2}\right), \\
\epsilon_{i}:=h_{i}-\tilde{h}_{i}, \\
\tilde{h}_{i}:=\frac{1}{M^{2}} n_{i},
\end{gathered}
$$

where $n_{i}:=\left\lfloor M^{2} h_{i}\right\rfloor$ and $h_{i}$ is the $i$ th eigenvalue of $A_{S}$. Note that $0 \leqslant \epsilon_{i}<1 / M^{2}$ holds by definition. We will define $\epsilon$ in the next subsection.

The pure initial state $\phi_{\zeta}^{L, M}$ of $E$ is given as follows:

$$
\phi_{\zeta}^{L, M}=\phi_{E_{0}}^{L, M, \zeta} \otimes\left(\otimes_{i=1}^{d_{S}} \phi_{E_{i}}^{L, M}\right) \otimes|1\rangle\left\langle\left. 1\right|_{S^{\prime}} \otimes \phi_{E_{d_{S}+1}},\right.
$$

where $|1\rangle_{S^{\prime}}$ is the first eigenstate of $\tilde{A}_{S^{\prime}}^{M}$ and

$$
\begin{aligned}
\left|\phi_{E_{0}}^{L, M, \zeta}\right\rangle:=C_{\zeta, L, M} & \frac{1}{2 \sqrt{L} M} \sum_{m \in B_{2 L}} e^{-\frac{x_{m}^{2}}{4 \zeta^{2}}}|m\rangle_{E_{0}}, \\
\left|\phi_{E_{i}}^{L, M}\right\rangle & :=\frac{1}{\sqrt{M}} \sum_{m=1}^{M}|m\rangle_{E_{i}}, \\
\left|\phi_{E_{d_{S}+1}}\right\rangle & :=\frac{|0\rangle_{E_{d_{S}+1}}+|1\rangle_{E_{d_{S}+1}}}{\sqrt{2}},
\end{aligned}
$$

where $C_{\zeta, L, M}$ is the normalized factor and

$$
B_{2 L}:=\left\{m|| x_{m} \mid<2 L\right\} .
$$

To construct $U_{S E}^{L, M}$, we introduce two types of unitary operations:

$$
\begin{aligned}
U_{S^{\prime} E_{0}}^{L, M}:= & \sum_{i} \sum_{(j, m) \in B} u_{i j}|i\rangle\left\langle\left. j\right|_{S^{\prime}} \otimes \mid m-n_{i}+n_{j}\right\rangle\left\langle\left. m\right|_{E_{0}}\right. \\
& +\sum_{(j, m) \notin B}|j\rangle\left\langle\left. j\right|_{S^{\prime}} \otimes \mid m\right\rangle\left\langle\left. m\right|_{E_{0^{\prime}}}\right. \\
B & :=\left\{(j, m) \mid-3 L \leqslant \tilde{h}_{j}+x_{m} \leqslant 3 L\right\},
\end{aligned}
$$

where $u_{i j}$ is defined as $u_{i j}:=\left\langle\left. i\right|_{S} U_{S} \mid j\right\rangle_{S}$ :

$$
\begin{aligned}
U_{S S^{\prime},\left\{E_{k}\right\}_{k=1}^{d_{S}}}^{L, M} & =\sum_{\left(i, j,\left\{m_{k}\right\}\right) \in C}|j\rangle\left\langle\left. i\right|_{S} \otimes \mid i\right\rangle\left\langle\left. j\right|_{S^{\prime}}\right. \\
& \otimes\left(\otimes_{k=1}^{d_{S}}\left|m_{k}+\delta_{k, i}-\delta_{k, j}\right\rangle\left\langle\left. m_{k}\right|_{E_{k}}\right)\right. \\
& +\sum_{(i, j, m) \notin C}|i\rangle\left\langle\left. i\right|_{S} \otimes \mid j\right\rangle\left\langle\left. j\right|_{S^{\prime}}\right. \\
& \otimes\left(\otimes_{k=1}^{d_{S}}\left|m_{k}\right\rangle\left\langle\left. m_{k}\right|_{E_{k}}\right),\right.
\end{aligned}
$$

$$
\begin{aligned}
C:= & \left\{\left(i, j,\left\{m_{k}\right\}_{k=1}^{d_{S}}\right) \mid\right. \\
& \left.-2 M \leqslant m_{k}+\delta_{k, i}-\delta_{k, j} \leqslant 2 M \text { for each } k\right\} .
\end{aligned}
$$

With using these two unitary operations, we construct $U_{S E}^{L, M}$ as follows:

$$
U_{S E}^{L, M}:=\left(U_{S S^{\prime}\left\{E_{d_{S}+k},\right.}^{L, M} U_{S^{\prime} E_{0}}^{L, M} U_{S S^{\prime}\left\{E_{k}\right\}}^{L, M}\right) \otimes 1_{E_{2 d_{S}+1}},
$$

where $U_{S S^{\prime}\left\{E_{d_{s}+k}\right\}}^{L, M}$ is a unitary operation defined by substituting $E_{d_{s}+k}$ for $E_{k}$ of (C20). In this section, we sometimes use the abbreviation of $U_{S^{\prime} E_{0}}^{L, M}, U_{S S^{\prime}\left\{E_{k}\right\}}^{L, M}$, and $U_{S S^{\prime}\left\{E_{k}\right\}}^{L, M}$ as $U_{0}, U_{\{k\}}$, and $U_{\left\{k+d_{S}\right\}}$, respectively.

Now, we have defined $\mathcal{I}_{\zeta, L, M, \epsilon}=\left(\mathcal{H}_{E}^{L, M}, A_{E}^{L, M, \epsilon}, \phi_{\zeta}^{L, M}\right.$, $U_{S E}^{L, M}$ ). To show that $\mathcal{I}_{\zeta, L, M, \epsilon}$ is well defined, we have to show the unitarity of $U_{S E}^{L, M}$ and $\left[U_{S E}^{L, M}, A_{S}+A_{E}^{L, M, \epsilon}\right]=0$. Due to

$$
\begin{gathered}
U_{0}, \tilde{A}_{S^{\prime}}^{M}+A_{E_{0}}^{L, M}=0, \\
U_{\{k\}}, A_{S}+\tilde{A}_{S^{\prime}}^{M}+\sum_{k=1}^{d_{S}} A_{E_{k}}^{L, M}=0, \\
U_{\left\{k+d_{S}\right\}}, A_{S}+\tilde{A}_{S^{\prime}}^{M}+\sum_{k=1}^{d_{S}} A_{E_{k+d_{S}}}^{L, M}=0,
\end{gathered}
$$

the relation $\left[U_{S E}^{L, M}, A_{S}+A_{E}^{L, M, \epsilon}\right]=0$ clearly holds. Below, we show the unitarity of $U_{S E}^{L, M}$.

Proof. the unitarity of $U_{S E}^{L, M}$ To show this, we only have to show the unitarity of $U_{0}$ and $U_{\{k\}}$, respectively. We first show the unitarity of $U_{0}$, i.e., $U_{0}^{\dagger} U_{0}=U_{0}^{\dagger} U_{0}=I$. Let us refer to the first and second summations in (C18) as $U_{0, A}$ and $U_{0, B}$, respectively. Due to $U_{0}=U_{0, A}+U_{0, B}$ and $U_{0, B}^{\dagger}=U_{0, B}=U_{0, B}^{2}$, we only have to show the following:

$$
\begin{gathered}
U_{0, A} U_{0, B}=U_{0, B} U_{0, A}=0, \\
U_{0, A}^{\dagger} U_{0, A}+U_{0, B}=U_{0, A} U_{0, A}^{\dagger}+U_{0, B}=I .
\end{gathered}
$$

To show these relations, we show the following relation:

$$
U_{0, A}=\sum_{i} \sum_{(j, \bar{m}) \in B} u_{j i}|j\rangle\left\langle\left. i\right|_{S^{\prime}} \otimes \mid \bar{m}\right\rangle\left\langle\bar{m}+n_{j}-\left.n_{i}\right|_{E_{0}} .\right.
$$

To obtain the relation $(\mathrm{C} 28)$, we only have to note the following relation:

$$
(j, m) \in B \Leftrightarrow\left(i, m-n_{i}+n_{j}\right) \in B .
$$

Therefore, by relabeling $\left(i, j, m-n_{i}+m_{j}\right)$ of the first term of (C18) to $(j, i, \bar{m})$, we obtain (C28). [Here, note that when $(j, m) \in B$ holds, the inequality $-3 L \leqslant x_{m+n_{j}} \leqslant 3 L$ holds by definition. Since $\left\|A_{S}\right\|<L$, the inequality $-4 L<x_{m-n_{i}+n_{j}}<$ $4 L$ always holds. Therefore, $\left|m-n_{i}+n_{j}\right\rangle_{E_{0}}$ always exists when $(j, m) \in B$ holds. Similarly, we can show that $|m\rangle_{E_{0}}$ always exists when $\left(i, m-n_{i}+n_{j}\right) \in B$ holds, and thus the above relation (C29) is well defined.]

Using the relation (C28) and the definition (C18), we obtain the following relation:

$$
U_{0, A}=\Pi_{B} U_{0, A}=U_{0, A} \Pi_{B},
$$

where $\Pi_{B}:=\sum_{(j, m) \in B}|j\rangle\left\langle\left. j\right|_{S^{\prime}} \otimes \mid m\right\rangle\left\langle\left. m\right|_{E_{0}}\right.$. Here, to derive $U_{0, A}=\Pi_{B} U_{0, A}$ in (C30), note that the left half of the 
right-hand side of $(\mathrm{C} 28)$ has the same form as the right half of the first term of the right-hand side of $(\mathrm{C} 18)$. Due to $(\mathrm{C} 30)$ and $U_{B}=I-\Pi_{B}$, we obtain (C26).

Next, we obtain (C27). Due to $U_{B}=I-\Pi_{B}$, we only have to show $U_{0, A}^{\dagger} U_{0, A}=U_{0, A} U_{0, A}^{\dagger}=\Pi_{B}$. By using (C18), we obtain

$$
\begin{aligned}
U_{0, A}^{\dagger} U_{0, A}= & \sum_{i, i^{\prime}} \sum_{(j, m) \in B} \sum_{\left(j^{\prime}, m^{\prime}\right) \in B} u_{i^{\prime} j^{\prime}}^{*} u_{i j}\left|j^{\prime}, m^{\prime}\right\rangle\left\langle j,\left.m\right|_{S^{\prime} E_{0}}\right. \\
& \times\left\langle i^{\prime}, m^{\prime}-n_{i^{\prime}}+n_{j^{\prime}} \mid i, m-n_{i}+n_{j}\right\rangle_{S^{\prime} E_{0}} \\
= & \sum_{i} \sum_{(j, m) \in B} \sum_{\left(j^{\prime}, m^{\prime}\right) \in B} u_{i j^{\prime}}^{*} u_{i j}\left|j^{\prime}, m^{\prime}\right\rangle\left\langle j,\left.m\right|_{S^{\prime} E_{0}}\right. \\
& \times \delta_{m^{\prime}+n_{j^{\prime}}, m+n_{j}} \\
= & \sum_{(j, m) \in B}|j, m\rangle\left\langle j,\left.m\right|_{S^{\prime} E_{0}}\right. \\
= & \Pi_{B} .
\end{aligned}
$$

Similarly, by using (C30), we obtain

$$
\begin{aligned}
U_{0, A} U_{0, A}^{\dagger}= & \sum_{j, j^{\prime}} \sum_{(i, \bar{m}) \in B} \sum_{\left(i^{\prime}, \bar{m}^{\prime}\right) \in B} u_{i^{\prime} j^{\prime}} u_{i j}^{*}\left|i^{\prime}, \bar{m}^{\prime}\right\rangle\left\langle i,\left.\bar{m}\right|_{S^{\prime} E_{0}}\right. \\
& \times\left\langle j^{\prime}, \bar{m}^{\prime}+n_{i^{\prime}}-n_{j^{\prime}} \mid j, \bar{m}+n_{i}-n_{j}\right\rangle_{S^{\prime} E_{0}} \\
= & \sum_{j} \sum_{(i, \bar{m}) \in B} \sum_{\left(i^{\prime}, \bar{m}^{\prime}\right) \in B} u_{i^{\prime} j^{\prime}} u_{i j}^{*}\left|i^{\prime}, m^{\prime}\right\rangle\left\langle i,\left.m\right|_{S^{\prime} E_{0}}\right. \\
& \times \delta_{\bar{m}^{\prime}+n_{i^{\prime}}, \bar{m}+n_{i}} \\
= & \sum_{(i, \bar{m}) \in B}|i, \bar{m}\rangle\left\langle i,\left.\bar{m}\right|_{S^{\prime} E_{0}}\right. \\
= & \Pi_{B} .
\end{aligned}
$$

Therefore, $U_{0}$ is unitary.

Next, we show the unitarity of $U_{\{k\}}$. We refer to the first and second summations in (C20) as $U_{\{k\}, A}$ and $U_{\{k\}, B}$, respectively. In the same manner as the proof of unitarity of $U_{0}$, we only have to show

$$
\begin{gathered}
U_{\{k\}, A} U_{\{k\}, B}=U_{\{k\}, B} U_{\{k\}, A}=0, \\
U_{\{k\}, A}^{\dagger} U_{\{k\}, A}+U_{\{k\}, B}=U_{\{k\}, A} U_{\{k\}, A}^{\dagger}+U_{\{k\}, B}=I .
\end{gathered}
$$

Again, we note the following relation:

$$
\begin{aligned}
U_{\{k\}, A}= & \sum_{\left(i, j,\left\{\bar{m}_{k}\right\}\right) \in C_{k}}|i\rangle\left\langle\left. j\right|_{S_{k-1}^{\prime}} \otimes \mid j\right\rangle\left\langle\left. i\right|_{S_{k}^{\prime}}\right. \\
& \otimes\left(\otimes_{k=1}^{d_{S}}\left|\bar{m}_{k}\right\rangle\left\langle\bar{m}_{k}+\delta_{k, i}-\left.\delta_{k, j}\right|_{E_{k}}\right) .\right.
\end{aligned}
$$

Therefore,

$$
U_{\{k\}, A}=U_{\{k\}, A} \Pi_{C}=U_{\{k\}, A} \Pi_{C}
$$

holds, and thus (C33) holds. (Here we define $\Pi_{C}:=$ $\sum_{\left(i, j,\left\{m_{k}\right\}\right) \in C}|i, j\rangle\left\langle i,\left.j\right|_{S, S^{\prime}} \otimes \otimes_{k=1}^{d_{S}} \mid m_{k}\right\rangle\left\langle\left. m_{k}\right|_{E_{k}}\right)$.

Finally, we show (C34). Due to $U_{\{k\}, B}=I-\Pi_{C}$, we only have to show $U_{\{k\}, A}^{\dagger} U_{\{k\}, A}=U_{\{k\}, A} U_{\{k\}, A}^{\dagger}=\Pi_{C}$. Due to (C20), we obtain $U_{\{k\}, A}^{\dagger} U_{\{k\}, A}=\Pi_{C}$. Due to (C35), we obtain $U_{\{k\}, A} U_{\{k\}, A}^{\dagger}=\Pi_{C}$.

\section{Three lemmas to prove Lemma 2}

Using $\mathcal{I}_{\zeta, L, M, \epsilon}$, we prove Lemma 2 . To prove it, we use the following three lemmas.
Lemma 4. There exist positive real numbers $b$ and $c$ such that the following inequalities hold for arbitrary enough large positive integers $L$ and $M$ :

$$
\begin{gathered}
\left|\left\langle\phi_{E_{0}, n_{h}}^{L, M, \zeta} \mid \phi_{E_{0},-n_{j}+n_{i}}^{L, M, \zeta}\right\rangle-e^{-\frac{\left(h_{i}-h_{j}-h\right)^{2}}{8 \zeta^{2}}}\right| \leqslant \frac{b}{L}+\frac{c}{M}, \\
\left|\left\langle\phi_{E_{i}}^{L, M} \mid \phi_{E_{i}, \pm 1}^{L, M}\right\rangle-1\right| \leqslant \frac{1}{M},
\end{gathered}
$$

where shifted $\left|\phi_{E_{0}}^{L, M, \zeta}\right\rangle$ and $\left|\phi_{E_{i}}^{L, M}\right\rangle$ are defined as follows:

$$
\begin{aligned}
&\left|\phi_{E_{0}, n}^{L, M, \zeta}\right\rangle:= C_{\zeta, L, M} \frac{1}{2 \sqrt{L} M} \sum_{m \in B_{2 L}} e^{-\frac{x_{m}^{2}}{4 \zeta^{2}}}|m+n\rangle_{E_{0}}, \\
&\left|\phi_{E_{i}, \pm 1}^{L, M}\right\rangle:=\frac{1}{\sqrt{M}} \sum_{m=1}^{M}|m \pm 1\rangle_{E_{i}}
\end{aligned}
$$

And we define $h$ as a real number satisfying $\| A_{S}-A_{S}^{\prime}-$ $h \hat{1} \|=\frac{\Delta_{A_{S}^{\prime}-A_{S}}}{2}=\mathcal{A}_{U_{S}}$ and also define

$$
\begin{aligned}
n_{h} & :=\left\lfloor h M^{2}\right\rfloor, \\
\tilde{h} & :=M^{2} n_{h} .
\end{aligned}
$$

Lemma 5. For $\zeta \geqslant 9 \mathcal{A}_{U_{S}} / 2 \sqrt{2}$, there exists a real positive number $r$, such that for any sufficiently large positive integers $L$ and $M$, the implementation set $\mathcal{I}_{\zeta, L, M, \epsilon}=$ $\left(\mathcal{H}_{E}^{L, M}, A_{E}^{L, M, \epsilon}, \phi_{\zeta}^{L, M}, U_{S E}^{L, M}\right)$ satisfies the following inequality for an arbitrary initial state $\rho_{S}$ on $S$ :

$$
\begin{aligned}
& F_{e}\left(\rho_{S}, \Lambda_{U_{S}^{\dagger}} \circ \Lambda_{S}^{\zeta, L, M}\right) \\
& \quad \geqslant\left|\left\langle T\left[e^{-\left(A_{S}-A_{S}^{\prime}-h \hat{\imath}\right)^{2} / 8 \zeta^{2}}\right]\right\rangle_{\rho_{S}}\right|\left(1-\frac{r d_{S}^{2}}{L}-\frac{r d_{S}^{2}}{M}\right),
\end{aligned}
$$

where we set $\Lambda_{S}^{\zeta, L, M}\left(\rho_{S}\right):=\operatorname{Tr}_{E}\left[U_{S E}^{L, M}\left(\rho_{S} \otimes \phi_{\zeta}^{L, M}\right) U_{S E}^{L, M \dagger}\right]$, $A_{S}^{\prime}:=U_{S}^{\dagger} A_{S} U_{S}$, and $h$ is a real number satisfying $\| A_{S}-$ $A_{S}^{\prime}-h \hat{1} \|=\frac{\Delta_{A_{S}^{\prime}-A_{S}}}{2}=\mathcal{A}_{U_{S}}$. The symbol $T$ represents the timeordering product (e.g., $T\left[A_{S}^{\prime} A_{S} A_{S}^{\prime}\right]=A_{S}^{\prime 2} A_{S}$ ).

Lemma 6 . We consider a quantum system, and take an arbitrary Hermitian $X$ and arbitrary unitary $U$ on the system. For $X$ and $X^{\prime}:=U^{\dagger} X U$, we define $X_{0}:=x_{0} \hat{1}$ such that $\| X-X^{\prime}-$ $X_{0} \|=\Delta_{X-X^{\prime}} / 2$. When $\left\|X-X^{\prime}-X_{0}\right\| \leqslant\|X\| \leqslant a$ holds for a positive number $a \leqslant 1 / 9$, the following inequality holds:

$$
\min _{\rho}\left|\left\langle T\left[e^{-\left(X-X^{\prime}-X_{0}\right)^{2}}\right]\right\rangle_{\rho}\right| \geqslant 1-\frac{\Delta_{X-X^{\prime}}^{2}}{4} \frac{e^{6 a}-1}{6 a} .
$$

Let us prove Lemmas 4-6.

Proof. Lemma 4 We first show (C37):

$$
\begin{aligned}
\left|\left\langle\phi_{E_{i}}^{L, M} \mid \phi_{E_{i}, \pm 1}^{L, M}\right\rangle-1\right| & =\left|\frac{1}{M} \sum_{m=1}^{M} \sum_{m^{\prime}=1}^{M}\left\langle m \mid m^{\prime} \pm 1\right\rangle-1\right| \\
& =\left|\frac{M-1}{M}-1\right|=\frac{1}{M}
\end{aligned}
$$


To show (C36), we note that

$$
\begin{aligned}
& \left\langle\phi_{E_{0},-n_{h}}^{L, M, \zeta} \mid \phi_{E_{0},-n_{j}+n_{i}}^{L, M, \zeta}\right\rangle \\
& =\frac{C_{\zeta, L, M}^{2}}{4 L M^{2}} \sum_{m^{\prime} \in B_{2 L}} \sum_{m \in B_{2 L}} e^{-\frac{x_{m^{\prime}}^{2}+x_{m}^{2}}{4 \zeta^{2}}} \delta_{m^{\prime}+n_{h}, m-n_{j}+n_{i}} \\
& =\frac{C_{\zeta, L, M}^{2}}{4 L M^{2}} \sum_{m \in B_{2 L}} e^{-\frac{x_{m+n_{i}-n_{j}-n_{h}}^{2}+x_{m}^{2}}{4 \zeta^{2}}}+o\left(e^{-L}\right) .
\end{aligned}
$$

We transform the first term of the right-hand side of $(\mathrm{C} 45)$ as follows:

$$
\begin{aligned}
& \frac{C_{\zeta, L, M}^{2}}{4 L M^{2}} \sum_{m \in B_{2 L}} e^{-\frac{x_{m+n_{i}-n_{j}-n_{h}+x_{m}^{2}}^{4 \zeta^{2}}}{4 L M^{2}}} \sum_{m \in B_{2 L}} e^{-\frac{\left(x_{m}+h_{i}-h_{j}+h+\gamma\right)^{2}+x_{m}^{2}}{4 \zeta^{2}}} \\
& =\frac{C_{\zeta, L, M}^{2}}{4 L M^{2}} \sum_{m \in B_{2 L}} e^{-\frac{2\left(x_{m}+\frac{h_{i}-h_{j}+h+\gamma}{2}\right)^{2}+\frac{\left(h_{i}-h_{j}+h+\gamma\right)^{2}}{2}}{4 \zeta^{2}}} \\
& =C_{\zeta, L, M}^{2} \\
& =e^{-\frac{\left(h_{i}-h_{j}+h+\gamma\right)^{2}}{8 \zeta^{2}}} \frac{C_{\zeta, L, M}^{2}}{4 L M^{2}} \sum_{m \in B_{2 L}} e^{-\frac{\left(x_{m}+\frac{h_{i}-h_{j}+h+\gamma}{2}\right)^{2}}{2 \zeta^{2}}},
\end{aligned}
$$

where $\gamma:=\tilde{h}_{i}-\tilde{h}_{j}+\tilde{h}-\left(h_{i}-h_{j}+h\right)$.

Let us evaluate the right-hand side of (C46). By defining $y_{m}:=x_{m}+\frac{\gamma}{2}$, and noting that $|\gamma|<2 / M^{2}$, we obtain

$$
\begin{aligned}
& \frac{C_{\zeta, L, M}^{2}}{4 L M^{2}} \sum_{m \in B_{2 L}} e^{-\frac{\left(x_{m}+\frac{h_{i}-h_{j}+h+\gamma}{2}\right)^{2}}{2 \zeta^{2}}} \\
& =\frac{C_{\zeta, L, M}^{2}}{4 L M^{2}} \sum_{m:-2 L-\gamma / 2 \leqslant y_{m} \leqslant 2 L-\gamma / 2} e^{-\frac{\left(y m+\frac{h_{i}-h_{j}+h}{2}\right)^{2}}{2 \zeta^{2}}} \\
& =\frac{\frac{1}{4 L M^{2}} \sum_{m:-2 L-\gamma / 2 \leqslant y_{m} \leqslant 2 L-\gamma / 2} e^{-\frac{\left(y m+\frac{h_{i}-h_{j}+h}{2}\right)^{2}}{2 \zeta^{2}}}}{\frac{1}{4 L M^{2}} \sum_{m:-2 L \leqslant x_{m} \leqslant 2 L} e^{-\frac{x_{m}^{2}}{2 \zeta^{2}}}} .
\end{aligned}
$$

To evaluate the right-hand side of (C47), we use the following relation for a smooth function $f$, which was given by Chui [76]:

$$
\begin{aligned}
& \frac{1}{n} \sum_{i=1}^{n} f\left(\frac{i(\beta-\alpha)}{n}-\frac{(\beta-\alpha)}{2 n}\right) \\
& \quad=\int_{\alpha}^{\beta} f(x) d x+\frac{(\beta-\alpha)^{2}}{24 n^{2}}\left[f^{\prime}(\beta)-f^{\prime}(\alpha)\right]+o\left(\frac{1}{n^{2}}\right) .
\end{aligned}
$$

When $\beta-\alpha$ is a positive integer taking $n=n^{\prime}(\beta-\alpha)$ with an integer $n^{\prime}$, we obtain the following relation from $(\mathrm{C} 48)$ :

$$
\begin{aligned}
& \frac{1}{n^{\prime}(\beta-\alpha)} \sum_{i=1}^{n^{\prime}(\beta-\alpha)} f\left(\frac{i}{n^{\prime}}-\frac{1}{2 n^{\prime}}\right) \\
& =\int_{\alpha}^{\beta} f(x) d x+\frac{1}{24 n^{\prime 2}}\left[f^{\prime}(\beta)-f^{\prime}(\alpha)\right]+o\left(\frac{1}{n^{\prime 2}}\right) .
\end{aligned}
$$

Due to (C49), we can take a positive number $r_{1}$ which is independent of $M$ and $L$ such that the following inequality is valid for any sufficiently large $M$ and $L$ :

$$
\begin{aligned}
& \left|\frac{1}{4 L M^{2}} \sum_{m:-2 L \leqslant x_{m} \leqslant 2 L} e^{-\frac{x_{m}^{2}}{2 \zeta^{2}}}-\int_{-2 L}^{2 L} e^{-\frac{x^{2}}{2 \zeta^{2}}} d x\right| \\
& \leqslant \frac{L}{6\left(M^{2}\right)^{2} \zeta^{2}} e^{-\frac{2 L^{2}}{\zeta^{2}}}+\frac{r_{1}}{M^{4}} .
\end{aligned}
$$

Here, we substitute $e^{-\frac{x^{2}}{2 \zeta^{2}}}, M^{2}, 2 L,-2 L$, and $m$ for $f(x), n^{\prime}, \beta$, $\alpha$, and $i$ in (C49).

Since $\quad \int_{-2 L}^{2 L} e^{-\frac{x^{2}}{2 \zeta^{2}}} d x=\int_{-\infty}^{\infty} e^{-\frac{x^{2}}{2 \zeta^{2}}} d x-2 \operatorname{Erfc}[2 L /(\sqrt{2} \zeta)]$ and $\operatorname{Erfc}(x)=o\left(e^{-x^{2}}\right)$, there exist positive numbers $r_{2}$ and $r_{3}$ which are independent of $M$ and $L$, and the following inequality holds for any sufficiently large $M$ and $L$ :

$$
\begin{aligned}
& \left|\frac{1}{4 L M^{2}} \sum_{m:-2 L \leqslant x_{m} \leqslant 2 L} e^{-\frac{x_{m}^{2}}{2 \zeta^{2}}}-\int_{-\infty}^{\infty} e^{-\frac{x^{2}}{2 \zeta^{2}}} d x\right| \\
& \leqslant \frac{r_{1}}{M^{4}}+r_{2} e^{-L}+\frac{r_{3} L e^{-L}}{M^{2}} .
\end{aligned}
$$

Similarly, there exist positive numbers $s_{1}, s_{2}$, and $s_{3}$ which are independent of $L$ and $M$ such that the following inequality holds for any sufficiently large $L$ and $M$ :

$$
\begin{aligned}
& \left|\frac{1}{4 L M^{2}} \sum_{-2 L-\gamma / 2 \leqslant y_{m} \leqslant 2 L-\gamma / 2} e^{-\frac{\left(y m+\frac{h_{i}-h_{j}+h}{2}\right)^{2}}{2 \zeta^{2}}}-\int_{-\infty}^{\infty} e^{-\frac{x^{2}}{2 \zeta^{2}}} d x\right| \\
& \leqslant \frac{s_{1}}{M^{4}}+s_{2} e^{-L}+\frac{s_{3} L e^{-L}}{M^{2}} .
\end{aligned}
$$

Now, we can evaluate the right-hand side of (C45). Due to $|\gamma|<1 / M^{2}$

$$
e^{-\frac{\left(h_{i}-h_{j}+h+\gamma\right)^{2}}{8 \zeta^{2}}}=e^{-\frac{\left(h_{i}-h_{j}+h\right)^{2}}{8 \zeta^{2}}}\left[1+O\left(\frac{1}{M^{2}}\right)\right] .
$$

Due to (C45), (C46), and (C51)-(C53), there exist $b, c>0$ which are independent of $L$ and $M$ such that the following inequality is valid for any sufficiently large $L$ and $M$ :

$$
\left|\left\langle\phi_{E_{0}, n_{h}}^{L, M, \zeta} \mid \phi_{E_{0},-n_{j}+n_{i}}^{L, M, \zeta}\right\rangle-e^{-\frac{\left(h_{i}-h_{j}-h\right)^{2}}{8 \zeta^{2}}}\right| \leqslant \frac{b}{L}+\frac{c}{M} .
$$

Proof. Lemma 5 We expand the initial state as

$$
\begin{gathered}
\rho_{S}:=\sum_{\lambda} p_{\lambda}\left|\psi_{S}^{(\lambda)}\right\rangle\left\langle\psi_{S}^{(\lambda)}\right|, \\
\left|\psi_{S}^{(\lambda)}\right\rangle:=\sum_{i} a_{i}^{(\lambda)}|i\rangle, \\
\left|\psi_{S R}\right\rangle:=\sum_{i} \sqrt{p^{(\lambda)}} a_{i}^{(\lambda)}|i\rangle|\lambda\rangle_{R} .
\end{gathered}
$$

Using these symbols, we can describe $F_{e}\left(\rho_{S}, \Lambda_{U_{S}^{\dagger}} \circ \Lambda_{S}\right)$ as follows:

$$
\begin{aligned}
& F_{e}\left(\rho_{S}, \Lambda_{U_{S}^{\dagger}} \circ \Lambda_{S}\right)^{2} \\
& \quad=\left\langle\psi_{S R}\left|\operatorname{Tr}_{E}\left[U_{S}^{\dagger} U_{S E}\left(\psi_{S R} \otimes \phi_{\zeta}\right) U_{S E}^{\dagger} U_{S}\right]\right| \psi_{S R}\right\rangle .
\end{aligned}
$$


By defining shifted $\phi_{\zeta, \tilde{h}}^{L, M}$ as

$$
\phi_{\zeta, \tilde{h}}^{L, M}:=\phi_{E_{0}, n_{h}}^{L, M, \zeta} \otimes\left(\otimes_{i=1}^{d_{S}} \phi_{E_{i}}^{L, M}\right) \otimes|1\rangle\left\langle\left. 1\right|_{S^{\prime}} \otimes \phi_{E_{d_{S}+1}},\right.
$$

we construct an orthonormal basis of $E$ as $\left\{\left|\phi_{\zeta, \tilde{h}}^{L, M}\right\rangle,\left|\phi_{E}^{(1)}\right\rangle, \ldots\right\}$. (Here, $\phi_{\zeta, \tilde{h}}^{L, M}$ depends on $\tilde{h}$ through $\tilde{h}=n_{h} M^{2}$ and $\phi_{E_{0}, n_{h}}^{L, M}$.) Expanding $\left|\phi_{E}\right\rangle$ with this basis, we find

$$
\begin{aligned}
F_{e}\left(\rho_{S}, \Lambda_{U_{S}^{\dagger}}^{L, M} \circ \Lambda_{S}\right)^{2}= & \left\langle\psi_{S R}\left|\operatorname{Tr}_{E}\left[U_{S}^{\dagger} U_{S E}\left(\psi_{S R} \otimes \phi_{\zeta, \tilde{h}}^{L, M}\right) U_{S E}^{\dagger} U_{S}\right]\right| \psi_{S R}\right\rangle \\
= & \left\langle\psi_{S R} \otimes \phi_{\zeta, \tilde{h}}^{L, M}\left|U_{S}^{\dagger} U_{S E}\left(\psi_{S R} \otimes \phi_{\zeta, \tilde{h}}^{L, M}\right) U_{S E}^{\dagger} U_{S}\right| \psi_{S R} \otimes \phi_{\zeta, \tilde{h}}^{L, M}\right\rangle \\
& +\left\langle\psi_{S R} \otimes \phi_{E}^{(1)}\left|U_{S}^{\dagger} U_{S E}\left(\psi_{S R} \otimes \phi_{\zeta, \tilde{h}}^{L, M}\right) U_{S E}^{\dagger} U_{S}\right| \psi_{S R} \otimes \phi_{E}^{(1)}\right\rangle+\ldots \\
\geqslant & \left\langle\psi_{S R} \otimes \phi_{\zeta, \tilde{h}}^{L, M}\left|U_{S}^{\dagger} U_{S E}\left(\psi_{S R} \otimes \phi_{\zeta, \tilde{h}}^{L, M}\right) U_{S E}^{\dagger} U_{S}\right| \psi_{S R} \otimes \phi_{\zeta, \tilde{h}}^{L, M}\right\rangle \\
= & \left.\| \psi_{S R} \otimes \phi_{\zeta, \tilde{h}}^{L, M}\left|U_{S}^{\dagger} U_{S E}\right| \psi_{S R} \otimes \phi_{\zeta, \tilde{h}}^{L, M}\right\rangle^{2},
\end{aligned}
$$

which is equivalent to

$$
F_{e}\left(\rho_{S}, \Lambda_{U_{S}^{\dagger}} \circ \Lambda_{S}^{L, M}\right) \geqslant\left|\left\langle\phi_{\zeta, \tilde{h}}^{L, M} \otimes \psi_{S R}\left|U_{S}^{\dagger} U_{S E}\right| \psi_{S R} \otimes \phi_{\zeta, \tilde{h}}^{L, M}\right\rangle\right| .
$$

To evaluate the right-hand side of (C61), we transform the right-hand side of (C61) as

$$
\begin{aligned}
& \left|\left\langle\phi_{\zeta, \tilde{h}}^{L, M} \otimes \psi_{S R}\left|U_{S}^{\dagger} U_{S E}\right| \psi_{S R} \otimes \phi_{\zeta, \tilde{h}}^{L, M}\right\rangle\right| \\
& \quad=\left|\sum_{\lambda} p_{\lambda} \sum_{i j k} a_{i}^{(\lambda)} u_{j i} a_{k}^{(\lambda) *} u_{j k}^{*} \cdot \Pi_{k=1}^{d_{S}}\left(\left\langle\phi_{E_{k}}^{L, M} \mid \phi_{E_{k},-\delta_{k, i}}^{L, M}\right\rangle\left\langle\phi_{E_{k+d_{S}}}^{L, M} \mid \phi_{E_{k+d_{S}},+\delta_{k, j}}^{L, M}\right\rangle\right) \cdot\left\langle\phi_{E_{0},-n_{h}}^{L, M, \zeta} \mid \phi_{E_{0},-n_{j}+n_{i}}^{L, M, \zeta}\right\rangle\right| .
\end{aligned}
$$

For arbitrary complex numbers $\left\{\alpha_{x}\right\}$ and real positive numbers $\left\{\beta_{x}\right\}$ and $\left\{\tilde{\beta}_{x}\right\}$ satisfying $\left|\beta_{x}-\tilde{\beta}_{x}\right|<\gamma(\gamma$ is a real positive number), the following inequality holds:

$$
\left|\sum_{x} \alpha_{x} \tilde{\beta}_{x}\right|>\left|\sum_{x} \alpha_{x} \beta_{x}\right|-\gamma \sum_{x}\left|\alpha_{x}\right| .
$$

Therefore, due to Lemma 4 and the fact $\left|\sum_{\lambda} p_{\lambda} \sum_{k} a_{i}^{(\lambda)} u_{j i} a_{k}^{(\lambda) *} u_{j k}^{*}\right| \leqslant 1$, there exists a real positive number $r$ which is independent of $L$ and $M$ such that the following inequality holds:

$$
\begin{aligned}
& \left|\left\langle\phi_{\zeta, \tilde{h}}^{L, M} \otimes \psi_{S R}\left|U_{S}^{\dagger} U_{S E}\right| \psi_{S R} \otimes \phi_{\zeta, \tilde{h}}^{L, M}\right\rangle\right| \\
& \quad \geqslant\left|\sum_{\lambda} p_{\lambda} \sum_{i j k} a_{i}^{(\lambda)} u_{j i} a_{k}^{(\lambda) *} u_{j k}^{*} e^{-\frac{\left(h_{i}-h_{j}-h\right)^{2}}{8 \zeta^{2}}}\right| \cdot\left[1-\left(\frac{r d_{S}^{2}}{M}+\frac{r d_{S}^{2}}{L}\right)\right] \\
& \quad=\left|\sum_{\lambda} p_{\lambda} \sum_{i j k} a_{i}^{(\lambda)} u_{j i} a_{k}^{(\lambda) *} u_{j k}^{*} \sum_{l=0}^{\infty} \frac{(-1)^{l}}{l !}\left(\frac{\left(h_{i}-h_{j}-h\right)^{2}}{8 \zeta^{2}}\right)^{l}\right| \cdot\left[1-\left(\frac{r d_{S}^{2}}{M}+\frac{r d_{S}^{2}}{L}\right)\right] .
\end{aligned}
$$

Finally, applying

$$
\sum_{i j k} a_{i}^{(\lambda)} u_{j i} a_{k}^{(\lambda) *} u_{j k}^{*}\left(\frac{\left(h_{i}-h_{j}-h\right)^{2}}{8 \zeta^{2}}\right)^{l}=\left\langle\psi_{S}^{(\lambda)}\left|T\left[\left(\frac{\left(A_{S}-A_{S}^{\prime}-h \hat{I}\right)^{2}}{8 \zeta^{2}}\right)^{l}\right]\right| \psi_{S}^{(\lambda)}\right\rangle
$$

to the right-hand side of (C64), we obtain

$$
\begin{aligned}
& \left|\sum_{\lambda} p_{\lambda} \sum_{i j k} a_{i}^{(\lambda)} u_{j i} a_{k}^{(\lambda) *} u_{j k}^{*} \sum_{l=0}^{\infty} \frac{(-1)^{l}}{l !}\left(\frac{\left(h_{i}-h_{j}-h\right)^{2}}{8 \zeta^{2}}\right)^{l}\right| \\
& \quad=\left|\sum_{\lambda} p_{\lambda}\left\langle\psi_{S}^{(\lambda)}\left|\sum_{l=0}^{\infty} \frac{(-1)^{l}}{l !} T\left[\left(\frac{\left(A_{S}-A_{S}^{\prime}-h \hat{I}\right)^{2}}{8 \zeta^{2}}\right)^{l}\right]\right| \psi_{S}^{(\lambda)}\right\rangle\right|=\left|\left\langle T\left[e^{-\left(A_{S}-A_{S}^{\prime}-h \hat{I}\right)^{2} / 8 \zeta^{2}}\right]\right\rangle_{\rho}\right| .
\end{aligned}
$$

In conclusion, we arrive at the desired inequality

$$
F_{e}\left(\rho_{S}, \Lambda_{U_{S}^{\dagger}} \circ \Lambda_{S}^{\zeta, L, M}\right) \geqslant \mid\left\langle T\left[e^{-\left(A_{S}-A_{S}^{\prime}-h \hat{1}\right)^{2} / 8 \zeta^{2}}\right]\right\rangle_{\rho_{S}}\left(1-\frac{r d_{S}^{2}}{L}-\frac{r d_{S}^{2}}{M}\right) .
$$


Proof. Lemma 6 The Taylor expansion of $T\left[e^{-\left(X-X^{\prime}-X_{0}\right)^{2}}\right]$ reads

$$
T\left[e^{-\left(X-X^{\prime}-X_{0}\right)^{2}}\right]=1-T_{2}+\frac{1}{2 !} T_{4}-\frac{1}{3 !} T_{6}+\ldots,
$$

where we employed the abbreviation $T_{m}:=T\left[\left(X-X^{\prime}-X_{0}\right)^{m}\right]$. Using this expression, we have the following inequality:

$$
\left|\left\langle T\left[e^{-\left(X-X^{\prime}-X_{0}\right)^{2}}\right]\right\rangle_{\rho}\right| \geqslant\left|\operatorname{Re}\left(\left\langle T\left[e^{-\left(X-X^{\prime}-X_{0}\right)^{2}}\right]\right\rangle_{\rho}\right)\right| \geqslant 1-\sum_{m=1}^{\infty} \frac{1}{m !}\left|\left\langle S_{2 m}\right\rangle_{\rho}\right| \geqslant 1-\sum_{m=1}^{\infty} \frac{1}{m !}\left\|S_{2 m}\right\|,
$$

where we denoted the Hermitian part of $T_{2 m}$ by $S_{2 m}:=\left(T_{2 m}+T_{2 m}^{\dagger}\right) / 2$. For convenience, we also define the anti-Hermitian part of $T_{s m}$ as $A_{2 m}$. The operator norms of $S_{2 m}$ and $A_{2 m}$ are bounded from above as

$$
\begin{gathered}
\left\|S_{2 m}\right\| \leqslant\left\|X-X^{\prime}-X_{0}\right\|^{2}(6 a)^{m-1}, \\
\left\|A_{2 m}\right\| \leqslant\left\|X-X^{\prime}-X_{0}\right\|(6 a)^{m-1} .
\end{gathered}
$$

We first see how (C70) leads to the desired inequality (C43), and then we prove the above inequalities. Using (C70), the left-hand side of (C43) is evaluated as

$$
\begin{aligned}
\min _{\rho}\left|\left\langle T\left[e^{-\left(X-X^{\prime}-X_{0}\right)^{2}}\right]\right\rangle_{\rho}\right| & \geqslant 1-\left\|X-X^{\prime}-X_{0}\right\|^{2}\left(1+\frac{1}{2 !}(6 a)+\frac{1}{3 !}(6 a)^{2}+\ldots\right) \\
& =1-\left\|X-X^{\prime}-X_{0}\right\|^{2} \frac{e^{6 a}-1}{6 a} \\
& =1-\frac{\Delta_{X-X^{\prime}}^{2}}{4} \frac{e^{6 a}-1}{6 a} .
\end{aligned}
$$

We now prove $(\mathrm{C} 70)$ by using the mathematical induction on $m$. We also prove $(\mathrm{C} 71)$ as a byproduct. In this proof, we put $Y:=X-X^{\prime}-X_{0}$ for brevity. We first show (C70) and (C71) for $m=1$. Recalling $T_{2}=T\left[\left(X-X^{\prime}-X_{0}\right)^{2}\right]$, we have

$$
S_{2}=Y^{2}, A_{2}=\left[X^{\prime}, X\right]=[Y, X]
$$

which directly imply

$$
\left\|S_{2}\right\|=\|Y\|^{2}, \quad\left\|A_{2}\right\| \leqslant 2 a\|Y\| \leqslant\|Y\| .
$$

Hence, (C70) and (C71) hold for $m=1$.

We next show the inductive step. Assume that (C70) and (C71) hold for $m \leqslant k$. We shall show that (C70) and (C71) also hold for $m=k+1$. Inserting the following recursion twice to the definition of $T_{2 m}$,

$$
T_{n}=T_{n-1} X-\left(X^{\prime}+X_{0}\right) T_{n-1}=\left[T_{n-1}, X\right]+Y T_{n-1},
$$

we obtain the recursion relation between $T_{2 n}$ and $T_{2(n-1)}$ :

$$
T_{2 n}=\left[T_{2 n-1}, X\right]+Y T_{2 n-1}=\left[\left[T_{2(n-1)}, X\right], X\right]+\left[Y T_{2(n-1)}, X\right]+Y\left[T_{2(n-1)}, X\right]+Y^{2} T_{2(n-1)} .
$$

Using the relations $T_{2(n-1)}=S_{2(n-1)}+A_{2(n-1)}, S_{2 n}=\frac{T_{2 n}+T_{2 n}^{\dagger}}{2}$ and $A_{2 n}=\frac{T_{2 n}-T_{2 n}^{\dagger}}{2}$, we divide this recursion into the ones about $S_{2 n}$ and $A_{2 n}$, respectively. From $T_{2(n-1)}=S_{2(n-1)}+A_{2(n-1)}$, we obtain

$$
\begin{aligned}
T_{2 n}= & {\left[\left[S_{2(n-1)}, X\right], X\right]+\left[Y S_{2(n-1)}, X\right]+Y\left[S_{2(n-1)}, X\right]+Y^{2} S_{2(n-1)}+\left[\left[A_{2(n-1)}, X\right], X\right]+\left[Y A_{2(n-1)}, X\right] } \\
& +Y\left[A_{2(n-1)}, X\right]+Y^{2} A_{2(n-1)} \\
= & {\left[\left[S_{2(n-1)}, X\right], X\right]+2 Y S_{2(n-1)} X-X Y S_{2(n-1)}-Y X S_{2(n-1)}+Y^{2} S_{2(n-1)}+\left[\left[A_{2(n-1)}, X\right], X\right]+2 Y A_{2(n-1)} X-X Y A_{2(n-1)} } \\
& -Y X A_{2(n-1)}+Y^{2} A_{2(n-1)} .
\end{aligned}
$$

From $S_{2 n}=\frac{T_{2 n}+T_{2 n}^{\dagger}}{2}$ and $A_{2 n}=\frac{T_{2 n}-T_{2 n}^{\dagger}}{2}$, the above equality reads

$$
\begin{aligned}
S_{2 n}= & {\left[\left[S_{2(n-1)}, X\right], X\right]+\frac{1}{2}\left(2 Y S_{2(n-1)} X-\{X, Y\} S_{2(n-1)}+Y^{2} S_{2(n-1)}\right)+\frac{1}{2}\left(2 X S_{2(n-1)} Y-S_{2(n-1)}\{X, Y\}+S_{2(n-1)} Y^{2}\right) } \\
& +\frac{1}{2}\left(2 Y A_{2(n-1)} X-\{X, Y\} A_{2(n-1)}+Y^{2} A_{2(n-1)}\right)-\frac{1}{2}\left(2 X A_{2(n-1)} Y-A_{2(n-1)}\{X, Y\}+A_{2(n-1)} Y^{2}\right) \\
A_{2 n}=[ & {\left.\left[A_{2(n-1)}, X\right], X\right]+\frac{1}{2}\left(2 Y S_{2(n-1)} X-\{X, Y\} S_{2(n-1)}+Y^{2} S_{2(n-1)}\right)-\frac{1}{2}\left(2 X S_{2(n-1)} Y-S_{2(n-1)}\{X, Y\}+S_{2(n-1)} Y^{2}\right) } \\
& +\frac{1}{2}\left(2 Y A_{2(n-1)} X-\{X, Y\} A_{2(n-1)}+Y^{2} A_{2(n-1)}\right)+\frac{1}{2}\left(2 X A_{2(n-1)} Y-A_{2(n-1)}\{X, Y\}+A_{2(n-1)} Y^{2}\right)
\end{aligned}
$$


By taking operator norms of the above relations, the following recursion relations are obtained:

$$
\begin{aligned}
\left\|S_{2 n}\right\| & \leqslant\left\|S_{2(n-1)}\right\| 4\|X\|^{2}+\left\|S_{2(n-1)}\right\|\left(4\|X\|\|Y\|+\|Y\|^{2}\right)\left|+\left\|A_{2(n-1)}\right\|\left(4\|X\|\|Y\|+\|Y\|^{2}\right)\right| \\
& \leqslant\left\|S_{2(n-1)}\right\| 9 a^{2}+\left\|A_{2(n-1)}\right\| 5 a\|Y\|,
\end{aligned}
$$

$\left\|A_{2 n}\right\| \leqslant\left\|A_{2(n-1)}\right\| 4\|X\|^{2}+\left\|S_{2(n-1)}\right\|\left(4\|X\|\|Y\|+\|Y\|^{2}\right)\left|+\left\|A_{2(n-1)}\right\|\left(4\|X\|\|Y\|+\|Y\|^{2}\right)\right| \leqslant\left\|A_{2(n-1)}\right\| 9 a^{2}+\left\|S_{2(n-1)}\right\| 5 a\|Y\|$.

Finally, using the induction hypothesis, we find that (C70) and (C71) hold for $m=k+1$ :

$$
\begin{gathered}
\left\|S_{2(k+1)}\right\| \leqslant\|Y\|^{2}(6 a)^{k-1} 9 a^{2}+\|Y\|(6 a)^{k-1} 5 a\|Y\| \leqslant\|Y\|^{2}(6 a)^{k}\left(\frac{9 a}{6}+\frac{5}{6}\right) \leqslant\|Y\|^{2}(6 a)^{k}, \\
\left\|A_{2(k+1)}\right\| \leqslant\|Y\|(6 a)^{k-1} 9 a^{2}+\|Y\|^{2}(6 a)^{k-1} 5 a\|Y\| \leqslant\|Y\|(6 a)^{k}\left(\frac{9 a}{6}+\frac{a}{6}\right) \leqslant\|Y\|(6 a)^{k} .
\end{gathered}
$$

By mathematical induction, (C70) and (C71) hold for any $m$.

\section{Proof of Lemma 2}

These two lemmas directly imply Lemma 2 as follows.

Proof. Lemma 2 Let us take $h^{\prime}$ such that the lowest eigenvalue of $A_{S}-h^{\prime} I_{S}$ is zero. Substituting $\left(A_{S}-h^{\prime} I_{S}\right) /(2 \sqrt{2} \zeta)$, $U_{S}$, and $\left\|A_{S}-h^{\prime} I_{S}\right\| /(2 \sqrt{2} \zeta)$ for $X, U$, and $a$ in (C43), we obtain

$$
\begin{aligned}
& \min _{\rho_{S}} F_{e}\left(\rho_{S}, \Lambda_{U_{S}^{\dagger}} \circ \Lambda_{S}^{\zeta, L, M}\right) \\
& \geqslant\left(1-\frac{\Delta_{A_{S}-A_{S}^{\prime}}^{2}}{32 \zeta^{2}} \frac{e^{6 a}-1}{6 a}\right)\left(1-\frac{d_{S}^{2} r}{L}-\frac{d_{S}^{2} r}{M}\right) \\
& =\left(1-\frac{\mathcal{A}_{U_{S}}^{2}}{8 \zeta^{2}} \frac{e^{6 a}-1}{6 a}\right)\left(1-\frac{d_{S}^{2} r}{L}-\frac{d_{S}^{2} r}{M}\right),
\end{aligned}
$$

where $a=\left\|A_{S}-h^{\prime} I_{S}\right\| /(2 \sqrt{2} \zeta)=\Delta_{A_{S}} /(2 \sqrt{2} \zeta)$.

Due to $\zeta \geqslant 9 \mathcal{A}_{U_{S}} / 2 \sqrt{2}$ and $\mathcal{A}_{U_{S}} \leqslant \Delta_{A_{S}}, a \leqslant 1 / 9$ holds, and thus

$$
\frac{e^{6 a}-1}{6 a}<(1+2 a)^{2} .
$$

Therefore, there exist enough large $L_{0}$ and $M_{0}$ such that for arbitrary $L>L_{0}$ and $M>M_{0}$ the following inequality holds:

$$
\min _{\rho_{S}} F_{e}\left(\rho_{S}, \Lambda_{U_{S}^{\dagger}} \circ \Lambda_{S}^{\zeta, L, M}\right) \geqslant 1-\frac{\mathcal{A}_{U_{S}}^{2}}{8 \zeta^{2}}(1+2 a)^{2} .
$$

Therefore, when $L>L_{0}$ and $M>M_{0}$ hold,

$$
\delta_{\mathcal{I}_{\zeta, L, M, \epsilon}} \leqslant \frac{\mathcal{A}_{U_{S}}}{2 \zeta}\left(1+\frac{\Delta_{A_{S}}}{\sqrt{2} \zeta}\right) .
$$

Now, let us give the implementation that we seek. We note that

$$
\lim _{M \rightarrow \infty} V_{\phi^{L, M, \zeta}}\left(A_{E_{0}}^{M, L}\right)=\mathcal{C}_{\zeta, L}^{2} \int_{-2 L}^{2 L} x^{2} e^{-\frac{x^{2}}{2 \zeta^{2}}} d x<\zeta^{2},
$$

where $\mathcal{C}_{\zeta, L}:=1 / \sqrt{\int_{-2 L}^{2 L} e^{-\frac{x^{2}}{2 \zeta^{2}} d x}}$. Hence, there exists a positive integer $M_{L, 0}$ depending on $L$ such that if $M>M_{L, 0}$,

$$
V_{\phi^{L, M, \zeta}}\left(A_{E_{0}}^{L, M}\right)<\zeta^{2}
$$

We also note that

$$
\sum_{i=1}^{d_{S}} V_{\phi_{E_{i}}^{L, M}}\left(A_{E_{i}}^{L, M}\right)<\sum_{i=1}^{d_{S}} \frac{1}{M^{2}}=\frac{d_{S}}{M^{2}} .
$$

Hence, there exists a positive integer $M_{L, 1}>M_{L, 0}$ such that if $M>M_{L, 1}$,

$$
V_{\phi^{L, M, \zeta}}\left(A_{E_{0}}^{M, L}\right)+\sum_{i=1}^{d_{S}} V_{\phi_{E_{i}}^{L, M}}\left(A_{E_{i}}^{L, M}\right)<\zeta^{2} .
$$

Therefore, we take $L>L_{0}$, and define $M_{L}$ as

$$
M_{L}:=\max \left\{M_{0}, M_{L, 1}\right\},
$$

and then define $\epsilon_{L}$ as

$$
\epsilon_{L}:=2 \sqrt{\zeta^{2}-V_{\phi_{E_{0}}^{L, M_{L}, \zeta}}\left(A_{E_{0}}^{L, M_{L}}\right)-\sum_{i=1}^{d^{s}} V_{\phi_{E_{i}}^{L, M_{L}}}\left(A_{E_{i}}^{L, M_{L}}\right) .}
$$

Let us define $\mathcal{I}_{\zeta, L}:=\mathcal{I}_{\zeta, L, M_{L}, \epsilon_{L}}$ and refer to its components as $\left(\mathcal{H}_{E}^{(L)}, A_{E}^{(L)}, \phi_{\zeta}^{(L)}, U_{S E}^{(L)}\right)$. Then, $\mathcal{I}_{\zeta, L}$ satisfies

$$
\begin{gathered}
\delta_{\mathcal{I}_{\zeta, L}} \leqslant \frac{\mathcal{A}_{U_{S}}}{2 \zeta}\left(1+\frac{\Delta_{A_{S}}}{\sqrt{2} \zeta}\right), \\
\mathcal{F}_{\phi_{\zeta}^{(L)}}\left(A_{E}^{(L)}\right)=4 V_{\phi_{E}^{(L)}}\left(A_{E}^{(L)}\right)=4 \zeta^{2} .
\end{gathered}
$$

\section{APPENDIX D: PROOF OF (22)}

Proof. (22) We first consider the case where both $\rho_{S}$ and $\rho_{E}$ are pure states. We here reshow some definitions of symbols used in this proof. We denote the desired final state and realized final state by

$$
\begin{aligned}
\left|\rho_{S}^{\prime}\right\rangle & :=U_{S}\left|\rho_{S}\right\rangle, \\
\left|\Psi_{S E}^{\prime}\right\rangle & :=U_{S E}\left|\rho_{S}\right\rangle\left|\rho_{E}\right\rangle, \\
\sigma_{E} & :=\operatorname{Tr}_{S}\left[\Psi_{S E}^{\prime}\right], \\
\sigma_{S} & :=\operatorname{Tr}_{E}\left[\Psi_{S E}^{\prime}\right] .
\end{aligned}
$$


In a similar manner, the final state of $\psi_{i}$ is denoted by

$$
\begin{aligned}
\left|\psi_{i}^{\prime}\right\rangle & :=U_{S}\left|\psi_{i}\right\rangle, \\
\left|\Psi_{i, S E}^{\prime}\right\rangle & :=U_{S E}\left|\psi_{i}\right\rangle\left|\rho_{E}\right\rangle, \\
\sigma_{i, E} & :=\operatorname{Tr}_{S}\left[\Psi_{i, S E}^{\prime}\right], \\
\sigma_{i, S} & :=\operatorname{Tr}_{E}\left[\Psi_{i, S E}^{\prime}\right] .
\end{aligned}
$$

Noting the definition of $\delta(\rho)$, Uhlmann's theorem leads to the fact that there are two pure states $\phi_{E}^{\prime}$ and $\phi_{i, E}^{\prime}$ satisfying

$$
\begin{aligned}
\left\langle\left\langle\Psi_{S E}^{\prime} \mid \rho_{S}^{\prime} \otimes \phi_{E}^{\prime}\right\rangle\right. & =\left(1-\frac{\delta\left(\rho_{S}\right)^{2}}{2}\right), \\
\mid\left\langle\Psi_{i, S E}^{\prime} \mid \psi_{i}^{\prime} \otimes \phi_{i, E}^{\prime}\right\rangle & =\left(1-\frac{\delta\left(\psi_{i}\right)^{2}}{2}\right),
\end{aligned}
$$

where we wrote $|\rho\rangle \otimes|\phi\rangle$ as $|\rho \otimes \phi\rangle$.

In the case of a pure state, $2 V_{\rho_{E}}\left(A_{E}\right)=\sqrt{\mathcal{F}_{\rho_{E}}\left(A_{E}\right)}$ holds by definition, and the pure state $\rho_{S}$ is written as $\left|\rho_{S}\right\rangle=\sum_{i} \alpha_{i}\left|\psi_{i}\right\rangle$. In this case, $r_{i}=\left|\alpha_{i}\right|^{2}$ holds, and the desired inequality (22) follows from

$$
\chi\left(\rho_{S},\left\{\psi_{i}\right\}\right)^{2} \leqslant 50 \bar{\delta}\left(\rho_{S},\left\{\psi_{i}\right\}\right)^{2}\left(V_{\rho_{E}}\left(A_{E}\right)+2 \Delta_{A_{S}}\right)^{2},
$$

where we used the abbreviation

$$
\bar{\delta}^{2}\left(\rho_{S},\left\{\psi_{i}\right\}\right):=\delta\left(\rho_{S}\right)^{2}+\sum_{i} r_{i} \delta\left(\psi_{i}\right)^{2} .
$$

Since the above inequality (D11) reduces to a trivial relation $V_{\rho_{E}}\left(A_{E}\right) \geqslant 0$ when $\bar{\delta}\left(\rho_{S},\left\{\psi_{i}\right\}\right)>\chi\left(\rho_{S},\left\{\psi_{i}\right\}\right)^{2} / 10 \sqrt{2} \Delta_{A_{S}}$ is satisfied, in the following we prove (D11) only for the case of $\bar{\delta}\left(\rho_{S},\left\{\psi_{i}\right\}\right) \leqslant \chi\left(\rho_{S},\left\{\psi_{i}\right\}\right)^{2} / 10 \sqrt{2} \Delta_{A_{S}}$.

We first employ (71) [the relation (c)], which is repeated below:

$$
\chi\left(\rho_{S},\left\{\psi_{i}\right\}\right) \leqslant \sqrt{\sum_{i} r_{i} \Delta_{i}^{2}}+\Delta^{\prime}+4 \bar{\delta}\left(\rho_{S}\right) \Delta_{A_{S}},
$$

where $\Delta_{i}:=\left|\left\langle A_{E}\right\rangle_{\sigma_{i, E}}-\left\langle A_{E}\right\rangle_{\phi_{E}^{\prime}}\right|$ and $\Delta^{\prime}:=\left|\left\langle A_{E}\right\rangle_{\sigma_{E}}-\left\langle A_{E}\right\rangle_{\phi_{E}^{\prime}}\right|$. The equation (D13) is shown as follows:

$$
\begin{aligned}
\chi\left(\rho_{S},\left\{\psi_{i}\right\}\right)^{2} & =\sum_{i} r_{i}\left(\left\langle A_{S}\right\rangle_{\psi_{i}}-\left\langle A_{S}\right\rangle_{U_{S} \psi_{i} U_{S}^{\dagger}}+\left\langle A_{S}\right\rangle_{U_{S} \rho_{S} U_{S}^{\dagger}}-\left\langle A_{S}\right\rangle_{\rho_{S}}\right)^{2} \\
& \leqslant \sum_{i} r_{i}\left\{\left|\left\langle A_{S}\right\rangle_{\psi_{i}}-\left\langle A_{S}\right\rangle_{\sigma_{i, S}}+\left\langle A_{S}\right\rangle_{\sigma_{S}}-\left\langle A_{S}\right\rangle_{\rho_{S}}\right|+2\left[\delta\left(\psi_{i}\right)+\delta\left(\rho_{S}\right)\right] \Delta_{A_{S}}\right\}^{2} \\
& =\sum_{i} r_{i}\left\{\left|\left\langle A_{E}\right\rangle_{\sigma_{i, E}}-\left\langle A_{E}\right\rangle_{\rho_{E}}+\left\langle A_{E}\right\rangle_{\rho_{E}}-\left\langle A_{E}\right\rangle_{\sigma_{E}}\right|+2\left[\delta\left(\psi_{i}\right)+\delta\left(\rho_{S}\right)\right] \Delta_{A_{S}}\right\}^{2} \\
& =\sum_{i} r_{i}\left\{\left|\left\langle A_{E}\right\rangle_{\sigma_{i, E}}-\left\langle A_{E}\right\rangle_{\sigma_{E}}\right|+2\left[\delta\left(\psi_{i}\right)+\delta\left(\rho_{S}\right)\right] \Delta_{A_{S}}\right\}^{2} \\
& \leqslant\left(\sqrt{\sum_{i} r_{i}\left(\left\langle A_{E}\right\rangle_{\sigma_{i, E}}-\left\langle A_{E}\right\rangle_{\sigma_{E}}\right)^{2}}+2 \sqrt{\sum_{i} r_{i}\left[\delta\left(\psi_{i}\right)+\delta\left(\rho_{S}\right)\right]^{2} \Delta_{A_{S}}^{2}}\right)^{2} \\
& \leqslant\left(\sqrt{\sum_{i} r_{i}\left(\left\langle A_{E}\right\rangle_{\sigma_{i, E}}-\left\langle A_{E}\right\rangle_{\phi_{E}^{\prime}}\right)^{2}}+\left|\left\langle A_{E}\right\rangle_{\sigma_{E}}-\left\langle A_{E}\right\rangle_{\phi_{E}^{\prime}}\right|+2 \sqrt{\sum_{i} r_{i}\left[\delta\left(\psi_{i}\right)+\delta\left(\rho_{S}\right)\right]^{2} \Delta_{A_{S}}^{2}}\right)^{2} \\
& =\left(\sqrt{\left.\sum_{i} r_{i} \Delta_{i}^{2}+\Delta^{\prime}+2 \sqrt{\sum_{i} r_{i}\left[\delta\left(\psi_{i}\right)+\delta\left(\rho_{S}\right)\right]^{2} \Delta_{A_{S}}^{2}}\right)^{2},}\right.
\end{aligned}
$$

where we used the conservation of $A$ in the total system in the third line. We obtain (D13) since the third term in the right-hand side of (D14) is easily bounded as

$$
\sum_{i} r_{i}\left[\delta\left(\psi_{i}\right)+\delta\left(\rho_{S}\right)\right]^{2} \Delta_{A_{S}}^{2} \leqslant 4 \bar{\delta}\left(\rho_{S},\left\{\psi_{i}\right\}\right)^{2} \Delta_{A_{S}}^{2} .
$$


Below we consider the first term in the right-hand side of (D14). This term is evaluated by using Lemma 1, or (74):

$$
\sum_{i} r_{i} \Delta_{i}^{2}=\sum_{i} r_{i}\left(\left\langle A_{E}\right\rangle_{\sigma_{i, E}}-\left\langle A_{E}\right\rangle_{\phi_{E}^{\prime}}\right)^{2} \leqslant \sum_{i} r_{i} L\left(\sigma_{i, E}, \phi_{E}^{\prime}\right)^{2}\left[V_{\sigma_{i, E}}\left(A_{E}\right)+V_{\phi_{E}^{\prime}}\left(A_{E}\right)+\Delta_{i}\right]^{2} .
$$

The first term in the bracket, $V_{\sigma_{i, E}}\left(A_{E}\right)$, is bounded as (78) [relation (b)]:

$$
V_{\sigma_{i, E}}\left(A_{E}\right) \leqslant V_{\rho_{E}}\left(A_{E}\right)+\Delta_{A_{S}} .
$$

In the subsequent analysis, we first bound the two correction terms, $V_{\phi_{E}^{\prime}}\left(A_{E}\right)$ and $\left|\left\langle A_{E}\right\rangle_{\sigma_{i, E}}-\left\langle A_{E}\right\rangle_{\phi_{E}^{\prime}}\right|$, by quantities independent of $i$. We then evaluate $\sum_{i} r_{i} L\left(\sigma_{i, E}, \phi_{E}^{\prime}\right)^{2}$ by using (76) [relation (a)].

We first derive the bound for $V_{\phi_{E}^{\prime}}\left(A_{E}\right)$. We compare the fluctuation of $A$ in $\rho_{S} \otimes \rho_{E}$ and $\rho_{S}^{\prime} \otimes \phi_{E}^{\prime}$ :

$$
\begin{aligned}
V_{\rho_{S} \otimes \rho_{E}}\left(A_{S}+A_{E}\right)^{2} & =V_{\Psi_{S E}^{\prime}}\left(A_{S}+A_{E}\right)^{2} \\
& =\operatorname{Tr}\left[\left(A_{S}+A_{E}-\left\langle A_{S}+A_{E}\right\rangle_{\Psi_{S E}^{\prime}}\right)^{2} \Psi_{S E}^{\prime}\right] \\
& \geqslant \operatorname{Tr}\left[\left(A_{S}+A_{E}-\left\langle A_{S}+A_{E}\right\rangle_{\Psi_{S E}^{\prime}}\right)^{2} P_{\rho_{S}^{\prime} \otimes \phi_{E}^{\prime}} \Psi_{S E}^{\prime} P_{\rho_{S}^{\prime} \otimes \phi_{E}^{\prime}}\right] \\
& =\operatorname{Tr}\left[\left(A_{S}+A_{E}-\left\langle A_{S}+A_{E}\right\rangle_{\Psi_{S E}^{\prime}}\right)^{2} \rho_{S}^{\prime} \otimes \phi_{E}^{\prime}\right] \operatorname{Tr}\left[P_{\rho_{S}^{\prime} \otimes \phi_{E}^{\prime}} \Psi_{S E}^{\prime}\right] \\
& =\operatorname{Tr}\left[\left(A_{S}+A_{E}-\left\langle A_{S}+A_{E}\right\rangle_{\Psi_{S E}^{\prime}}\right)^{2} \rho_{S}^{\prime} \otimes \phi_{E}^{\prime}\right]\left(1-\frac{\delta\left(\rho_{S}\right)^{2}}{2}\right)^{2} \\
& \geqslant V_{\rho_{S}^{\prime} \otimes \phi_{E}^{\prime}}\left(A_{S}+A_{E}\right)^{2}\left(1-\frac{\delta\left(\rho_{S}\right)^{2}}{2}\right)^{2} .
\end{aligned}
$$

Here, $P_{\rho_{S}^{\prime} \otimes \phi_{E}^{\prime}}:=\left|\rho_{S}^{\prime} \otimes \phi_{E}^{\prime}\right\rangle\left\langle\rho_{S}^{\prime} \otimes \phi_{E}^{\prime}\right|$ is the projection operator onto $\rho_{S}^{\prime} \otimes \phi_{E}^{\prime}$. By substituting $V_{\rho_{S} \otimes \rho_{E}}\left(A_{S}+A_{E}\right)^{2}=V_{\rho_{S}}\left(A_{S}\right)^{2}+$ $V_{\rho_{E}}\left(A_{E}\right)^{2} \leqslant \Delta_{A_{S}}^{2} / 4+V_{\rho_{E}}\left(A_{E}\right)^{2}$ and $V_{\rho_{S}^{\prime} \otimes \phi_{E}^{\prime}}\left(A_{S}+A_{E}\right)^{2}=V_{\rho_{S}^{\prime}}\left(A_{S}\right)^{2}+V_{\phi_{E}^{\prime}}\left(A_{E}\right)^{2} \geqslant V_{\phi_{E}^{\prime}}\left(A_{E}\right)^{2}$, we arrive at an upper bound for $V_{\phi_{E}^{\prime}}\left(A_{E}\right)$ :

$$
V_{\phi_{E}^{\prime}}\left(A_{E}\right) \leqslant \frac{\sqrt{\frac{1}{4} \Delta_{A_{S}}^{2}+V_{\rho_{E}}\left(A_{E}\right)^{2}}}{1-\frac{\delta\left(\rho_{S}\right)^{2}}{2}} .
$$

We next derive the bound for $\Delta_{i}:=\left|\left\langle A_{E}\right\rangle_{\sigma_{i, E}}-\left\langle A_{E}\right\rangle_{\phi_{E}^{\prime}}\right|$. We evaluate $\left|\left\langle A_{E}\right\rangle_{\sigma_{i, E}}-\left\langle A_{E}\right\rangle_{\phi_{E}^{\prime}}\right|$ as

$$
\left|\left\langle A_{E}\right\rangle_{\sigma_{i, E}}-\left\langle A_{E}\right\rangle_{\phi_{E}^{\prime}}\right| \leqslant\left|\left\langle A_{E}\right\rangle_{\sigma_{i, E}}-\left\langle A_{E}\right\rangle_{\sigma_{E}}\right|+\left|\left\langle A_{E}\right\rangle_{\sigma_{E}}-\left\langle A_{E}\right\rangle_{\phi_{E}^{\prime}}\right|=\left|\left\langle A_{S}\right\rangle_{\psi_{i}}-\left\langle A_{S}\right\rangle_{\sigma_{i, S}}-\left\langle A_{S}\right\rangle_{\sigma_{S}}+\left\langle A_{S}\right\rangle_{\rho_{S}}\right|+\Delta^{\prime} \leqslant 2 \Delta_{A_{S}}+\Delta^{\prime} \text {. }
$$

From Lemma 1 and $L\left(\sigma_{E}, \phi_{E}^{\prime}\right) \leqslant \delta\left(\rho_{S}\right)$, we derive the bound for $\Delta^{\prime}$ as follows:

$$
\Delta^{\prime} \leqslant \frac{L\left(\sigma_{E}, \phi_{E}^{\prime}\right)}{1-L\left(\sigma_{E}, \phi_{E}^{\prime}\right)}\left[V_{\sigma_{E}}\left(A_{E}\right)+V_{\phi_{E}^{\prime}}\left(A_{E}\right)\right] \leqslant \frac{\delta\left(\rho_{S}\right)}{1-\delta\left(\rho_{S}\right)}\left(V_{\rho_{E}}\left(A_{E}\right)+\Delta_{A_{S}}+\frac{\sqrt{\frac{1}{4} \Delta_{A_{S}}^{2}+V_{\rho_{E}}\left(A_{E}\right)^{2}}}{1-\frac{\delta\left(\rho_{S}\right)^{2}}{2}}\right) .
$$

At present, we have an upper bound for the right-hand side of (D16) as

$$
\begin{aligned}
& \sum_{i} r_{i} L\left(\sigma_{i, E}, \phi_{E}^{\prime}\right)^{2}\left[V_{\sigma_{i, E}}\left(A_{E}\right)+V_{\phi_{E}^{\prime}}\left(A_{E}\right)+\Delta_{i}\right]^{2} \\
& \quad \leqslant\left[\frac{1}{1-\delta\left(\rho_{S}\right)} V_{\rho_{E}}\left(A_{E}\right)+\frac{3-2 \delta\left(\rho_{S}\right)}{1-\delta\left(\rho_{S}\right)} \Delta_{A_{S}}+\frac{1}{1-\delta\left(\rho_{S}\right)} \frac{\sqrt{\frac{1}{4} \Delta_{A_{S}}^{2}+V_{\rho_{E}}\left(A_{E}\right)^{2}}}{1-\frac{\delta\left(\rho_{S}\right)^{2}}{2}}\right]^{2} \sum_{i} r_{i} L\left(\sigma_{i, E}, \phi_{E}^{\prime}\right)^{2} .
\end{aligned}
$$

We finally calculate the bound for $\sum_{i} r_{i} L\left(\sigma_{i, E}, \phi_{E}^{\prime}\right)^{2}$. Using a relation $\operatorname{Tr}[A B] \geqslant \operatorname{Tr}[\rho A \rho B]$ for positive Hermitian operators $A, B$ and a density matrix $\rho$ repeatedly, we have

$$
\begin{aligned}
r_{i}\left\langle\phi_{E}^{\prime}\left|\sigma_{i, E}\right| \phi_{E}^{\prime}\right\rangle & =\operatorname{Tr}\left[\left(\rho_{S}^{\prime} \otimes \phi_{E}^{\prime}\right)\left(\psi_{i}^{\prime} \otimes \sigma_{i, E}\right)\right] \\
& \geqslant \operatorname{Tr}\left[\Psi_{S E}^{\prime}\left(\rho_{S}^{\prime} \otimes \phi_{E}^{\prime}\right) \Psi_{S E}^{\prime}\left(\psi_{i}^{\prime} \otimes \sigma_{i, E}\right)\right] \\
& =\operatorname{Tr}\left[\Psi_{S E}^{\prime}\left(\rho_{S}^{\prime} \otimes \phi_{E}^{\prime}\right)\right] \operatorname{Tr}\left[\Psi_{S E}^{\prime}\left(\psi_{i}^{\prime} \otimes \sigma_{i, E}\right)\right] \\
& \geqslant \operatorname{Tr}\left[\Psi_{S E}^{\prime}\left(\rho_{S}^{\prime} \otimes \phi_{E}^{\prime}\right)\right] \operatorname{Tr}\left[\Psi_{S E}^{\prime} \Psi_{i, S E}^{\prime}\left(\psi_{i}^{\prime} \otimes \sigma_{i, E}\right) \Psi_{i, S E}^{\prime}\right] \\
& =\operatorname{Tr}\left[\Psi_{S E}^{\prime}\left(\rho_{S}^{\prime} \otimes \phi_{E}^{\prime}\right)\right] \operatorname{Tr}\left[\Psi_{S E}^{\prime} \Psi_{i, S E}^{\prime}\right] \operatorname{Tr}\left[\left(\psi_{i}^{\prime} \otimes \sigma_{i, E}\right) \Psi_{i, S E}^{\prime}\right] \\
& \geqslant \operatorname{Tr}\left[\Psi_{S E}^{\prime}\left(\rho_{S}^{\prime} \otimes \phi_{E}^{\prime}\right)\right] \operatorname{Tr}\left[\Psi_{S E}^{\prime} \Psi_{i, S E}^{\prime}\right] \operatorname{Tr}\left[\left(\psi_{i}^{\prime} \otimes \phi_{i, E}^{\prime}\right)\left(\psi_{i}^{\prime} \otimes \sigma_{i, E}\right)\left(\psi_{i}^{\prime} \otimes \phi_{i, E}^{\prime}\right) \Psi_{i, S E}^{\prime}\right]
\end{aligned}
$$




$$
\begin{aligned}
& =\operatorname{Tr}\left[\Psi_{S E}^{\prime}\left(\rho_{S}^{\prime} \otimes \phi_{E}^{\prime}\right)\right] \operatorname{Tr}\left[\Psi_{S E}^{\prime} \Psi_{i, S E}^{\prime}\right] \operatorname{Tr}\left[\left(\psi_{i}^{\prime} \otimes \phi_{i, E}^{\prime}\right)\left(\psi_{i}^{\prime} \otimes \sigma_{i, E}\right)\right] \operatorname{Tr}\left[\left(\psi_{i}^{\prime} \otimes \phi_{i, E}^{\prime}\right) \Psi_{i, S E}^{\prime}\right] \\
& =\operatorname{Tr}\left[\Psi_{S E}^{\prime}\left(\rho_{S}^{\prime} \otimes \phi_{E}^{\prime}\right)\right] \operatorname{Tr}\left[\Psi_{S E}^{\prime} \Psi_{i, S E}^{\prime}\right] \operatorname{Tr}\left[\left(\psi_{i}^{\prime} \otimes \phi_{i, E}^{\prime}\right) \Psi_{i, S E}^{\prime}\right] \operatorname{Tr}\left[\phi_{i, E}^{\prime} \sigma_{i, E}\right] \\
& \geqslant r_{i}\left(1-\frac{\delta\left(\rho_{S}\right)^{2}}{2}\right)^{2}\left(1-\frac{\delta\left(\psi_{i}\right)^{2}}{2}\right)^{4} .
\end{aligned}
$$

We combine $\left\langle\phi_{E}^{\prime}\left|\sigma_{i, E}\right| \phi_{E}^{\prime}\right\rangle=\left(1-L\left(\phi_{E}^{\prime}, \sigma_{i, E}\right)^{2} / 2\right)^{2}$ and

$$
\begin{aligned}
& \sum_{i} r_{i}\left(1-\frac{\delta\left(\rho_{S}\right)^{2}}{2}\right)^{2}\left(1-\frac{\delta\left(\psi_{i}\right)^{2}}{2}\right)^{4} \\
& \geqslant\left(1-\frac{\delta\left(\rho_{S}\right)^{2}}{2}\right)^{2}\left(1-\frac{\sum_{i} r_{i} \delta\left(\psi_{i}\right)^{2}}{2}\right)^{4},
\end{aligned}
$$

which follows from Jensen's inequality, noting $0 \leqslant 1-$ $L\left(\phi_{E}^{\prime}, \sigma_{i, E}\right)^{2} / 2 \leqslant 1$, and obtain

$$
1-\frac{\sum_{i} r_{i} L\left(\phi_{E}^{\prime}, \sigma_{i, E}\right)^{2}}{2} \geqslant 1-\left[\delta\left(\rho_{S}\right)^{2}+2 \sum_{i} r_{i} \delta\left(\psi_{i}\right)^{2}\right],
$$

which directly implies the desired bound

$$
\begin{aligned}
\sum_{i} r_{i} L\left(\phi_{E}^{\prime}, \sigma_{i, E}\right)^{2} & \leqslant 2\left[\delta\left(\rho_{S}\right)^{2}+2 \sum_{i} r_{i} \delta\left(\psi_{i}\right)^{2}\right] \\
& \leqslant 4 \bar{\delta}\left(\rho_{S},\left\{\psi_{i}\right\}\right)^{2} .
\end{aligned}
$$

In summary, by substituting all the obtained results into the right-hand side of (D14) and noting $\delta\left(\rho_{S}\right) \leqslant \bar{\delta}\left(\rho_{S},\left\{\psi_{i}\right\}\right)$, we obtain the following inequality:

$$
\begin{aligned}
\chi\left(\rho_{S},\left\{\psi_{i}\right\}\right) \leqslant & 2 \bar{\delta}\left(\rho_{S},\left\{\psi_{i}\right\}\right) \\
& \times\left[\frac{1.5}{1-\delta\left(\rho_{S}\right)} V_{\rho_{E}}\left(A_{E}\right)+\frac{5.5-4 \delta\left(\rho_{S}\right)}{1-\delta\left(\rho_{S}\right)} \Delta_{A_{S}}\right. \\
& \left.+\frac{1.5}{1-\delta\left(\rho_{S}\right)} \frac{\sqrt{\frac{1}{4} \Delta_{A_{S}}^{2}+V_{\rho_{E}}\left(A_{E}\right)^{2}}}{1-\frac{\delta\left(\rho_{S}\right)^{2}}{2}}\right] . \quad \text { (D27) }
\end{aligned}
$$

We note the inequality $\delta\left(\rho_{S}\right) \leqslant 1 / 10 \sqrt{2}$, which follows from the condition $\bar{\delta}\left(\rho_{S},\left\{\psi_{i}\right\}\right) \leqslant \chi\left(\rho_{S},\left\{\psi_{i}\right\}\right) / 10 \sqrt{2} \Delta_{A_{S}} \leqslant$ $1 / 10 \sqrt{2}$. Then, using the relation $\sqrt{\frac{1}{4} \Delta_{A_{S}}^{2}+V_{\rho_{E}}\left(A_{E}\right)^{2}} \leqslant$ $\frac{\Delta_{A_{S}}}{2}+V_{\rho_{E}}\left(A_{E}\right)$, we obtain

$$
\begin{aligned}
& \chi\left(\rho_{S},\left\{\psi_{i}\right\}\right) \\
& \quad \leqslant \bar{\delta}\left(\rho_{S},\left\{\psi_{i}\right\}\right)\left[6.464 \ldots \times V_{\rho_{E}}\left(A_{E}\right)+12.84 \ldots \times \Delta_{A_{S}}\right] \\
& \quad \leqslant \bar{\delta}\left(\rho_{S},\left\{\psi_{i}\right\}\right)\left[5 \sqrt{2} V_{\rho_{E}}\left(A_{E}\right)+10 \sqrt{2} \Delta_{A_{S}}\right],
\end{aligned}
$$

which readily implies (D11) for the case where both $\rho_{S}$ and $\rho_{E}$ are pure states.

Next, we consider the case where $\rho_{S}$ is mixed and $\rho_{E}$ is pure. We take a purification of $\rho_{S}$ as $\left|\psi_{S R}\right\rangle:=$ $\sum_{\lambda, i} \sqrt{p_{\lambda}}|\lambda\rangle\left|\rho_{\lambda}\right\rangle$. We expand each pure state $\rho_{\lambda}$ with the orthogonal basis $\left\{\psi_{i}\right\}$ as $\left|\rho_{\lambda}\right\rangle=\sum_{i} \alpha_{i}^{(\lambda)}\left|\psi_{i}\right\rangle$. Then, $\left|\psi_{S R}\right\rangle$ is rewritten as

$$
\left|\psi_{S R}\right\rangle=\sum_{i, \lambda} \sqrt{p_{\lambda}} \alpha_{i}^{(\lambda)}|\lambda\rangle\left|\psi_{i}\right\rangle
$$

By setting $\left\{\sqrt{p_{\lambda}} \alpha_{i}^{(\lambda)}\right\}$ and $\left\{|\lambda\rangle\left|\psi_{i}\right\rangle\right\}$ to $\left\{\alpha_{i}\right\}$ and $\left\{\left|\psi_{i}\right\rangle\right\}$ in the derivation of (D28) for pure $\rho_{S}$ and $\rho_{E}$, we obtain (D28) in this case. Therefore, we obtain (D11) in the case where $\rho_{E}$ is a pure state.

Finally, we show (D11) for the case where $\rho_{E}$ is a mixed state. We prove this in a similar manner to the proof of Theorem 1 in the case where $\rho_{E}$ is a mixed state. We employ the decomposition of $\rho_{E}$ into pure states $\left\{\rho_{\eta}\right\}$ (i.e., $\rho_{E}=$ $\left.\sum_{\eta} q_{\eta} \rho_{\eta}\right)$ as satisfying $\mathcal{F}_{\rho_{E}}\left(A_{E}\right)=4 \sum_{\eta} q_{\eta} V_{\rho_{\eta}}\left(A_{E}\right)^{2}$. We denote quantities $\bar{\delta}\left(\rho_{S},\left\{\psi_{i}\right\}\right), \delta\left(\rho_{S}\right)$, and $\delta\left(\psi_{i}\right)$, with the initial state of $E$ as $\rho_{\eta}$ by putting the subscript $\eta$ such as $\bar{\delta}\left(\rho_{S},\left\{\psi_{i}\right\}\right)_{\eta}$, $\delta\left(\rho_{S}\right)_{\eta}$, and $\delta\left(\psi_{i}\right)_{\eta}$.

Since (D28) has already been proven for a pure $\rho_{E}$, we have that the following inequality holds for each $\rho_{\eta}$ :

$$
V_{\rho_{\eta}}\left(A_{E}\right) \geqslant \frac{\chi\left(\rho_{S},\left\{\psi_{i}\right\}\right)}{5 \sqrt{2} \times \bar{\delta}\left(\rho_{S},\left\{\psi_{i}\right\}\right)_{\eta}}-2 \Delta_{A_{S}},
$$

where $\bar{\delta}\left(\rho_{S},\left\{\psi_{i}\right\}\right)_{\eta}$ has the following expression:

$$
\bar{\delta}\left(\rho_{S},\left\{\psi_{i}\right\}\right)_{\eta}=\sqrt{\delta\left(\psi_{i}\right)_{\eta}^{2}+\sum_{i} r_{i} \delta\left(\rho_{S}\right)_{\eta}^{2}}
$$

with $r_{i}:=\sum_{\lambda} p_{\lambda}\left|\alpha_{i}^{(\lambda)}\right|^{2}$. With keeping (D30) in mind, $V_{\rho_{E}}\left(A_{E}\right)$ is evaluated with a downward convex function $l(x):=\left(\max \left\{0, \frac{\chi\left(\rho_{S},\left\{\psi_{i}\right\}\right)}{5 \sqrt{2} x}-2 \Delta_{A_{S}}\right\}\right)^{2}(x>0)$ as

$$
\begin{aligned}
\frac{1}{4} \mathcal{F}_{\rho_{E}}\left(A_{E}\right) & =\sum_{\eta} q_{\eta} V_{\rho_{\eta}}\left(A_{E}\right)^{2} \\
& \geqslant \sum_{\eta} q_{\eta} l\left[\bar{\delta}\left(\rho_{S},\left\{\psi_{i}\right\}\right)_{\eta}\right] \\
& \geqslant l\left[\sum_{\eta} q_{\eta} \bar{\delta}\left(\rho_{S},\left\{\psi_{i}\right\}\right)_{\eta}\right] .
\end{aligned}
$$

Here, we shall prove

$$
\sum_{\eta} q_{\eta} \bar{\delta}\left(\rho_{S},\left\{\psi_{i}\right\}\right)_{\eta} \leqslant \sqrt{2} \times \bar{\delta}\left(\rho_{S},\left\{\psi_{i}\right\}\right) .
$$

We start from the following simple inequality:

$$
\begin{aligned}
\sum_{\eta} q_{\eta} \bar{\delta}\left(\rho_{S},\left\{\psi_{i}\right\}\right)_{\eta} & \leqslant \sqrt{\sum_{\eta} q_{\eta} \bar{\delta}\left(\rho_{S},\left\{\psi_{i}\right\}\right)_{\eta}^{2}} \\
& =\sqrt{\sum_{\eta} q_{\eta} \delta\left(\rho_{S}\right)_{\eta}^{2}+\sum_{\eta, i} q_{\eta} r_{i} \delta\left(\psi_{i}\right)_{\eta}^{2}} .
\end{aligned}
$$


Here, the following inequality holds for any $\rho$ :

$$
\sum_{\eta} q_{\eta} \delta(\rho)_{\eta}^{2} \leqslant 2 \delta(\rho)^{2},
$$

which is proven as follows. We denote the purification of $\rho$ by $|\psi S R\rangle$, and we define $\sigma_{S R}:=\Lambda_{S}\left(\psi_{S R}\right)$ and $\sigma_{S R, \eta}$ as that with the initial state of $E$ as $\rho_{\eta}$. We then have

$$
\begin{aligned}
\left(1-\frac{[\delta(\rho)]^{2}}{2}\right)^{2} & =\left\langle\psi_{S R}\left|U_{S}^{\dagger} \sigma_{S R} U_{S}\right| \psi_{S R}\right\rangle \\
& =\sum_{\eta} q_{\eta}\left\langle\psi_{S R}\left|U_{S}^{\dagger} \sigma_{S R, \eta} U_{S}\right| \psi_{S R}\right\rangle \\
& =\sum_{\eta} q_{\eta}\left(1-\frac{\delta(\rho)_{\eta}^{2}}{2}\right)^{2} \\
& \leqslant\left(1-\frac{\sum_{\eta} q_{\eta} \delta(\rho)_{\eta}^{2}}{2}\right)^{2} \\
& \leqslant\left(1-\frac{\sum_{\eta} q_{\eta} \delta(\rho)_{\eta}^{2}}{4}\right)^{2},
\end{aligned}
$$

which directly implies (D35). Applying (D35) to (D34), we arrive at the relation (D33).

Finally, substituting (D33) into (D32), along with the nonincreasingness of $l(x)$, we obtain

$$
\begin{aligned}
\sqrt{\mathcal{F}_{\rho_{E}}\left(A_{E}\right)} & \geqslant 2 \sqrt{l\left[\sum_{\eta} q_{\eta} \bar{\delta}\left(\rho_{S},\left\{\psi_{i}\right\}\right)_{\eta}\right]} \\
& \geqslant 2 \sqrt{l\left[\sqrt{2} \times \bar{\delta}\left(\rho_{S},\left\{\psi_{i}\right\}\right)\right]} \\
& =\frac{\chi\left(\rho_{S},\left\{\psi_{i}\right\}\right)}{5 \bar{\delta}\left(\rho_{S}\right)}-4 \Delta_{A_{S}},
\end{aligned}
$$

which readily implies the desired result (D11).

\section{APPENDIX E: A GENERALIZED VERSION OF THEOREM 1 FOR THE CASE WHERE $A_{S}+A_{E}$ IS NOT CONSERVED}

In this section, we consider the case that the total dynamics is unitary, but the quantity $A$ is not conserved perfectly, i.e., the case of $\left[U_{S E}, A_{S}+A_{E}\right] \neq 0$. In this case, we can define the degree of asymmetry of $U_{S E}$ :

$$
\mathcal{A}_{U_{S E}}:=\frac{\Delta_{i\left[U_{S E}, A_{S}+A_{E}\right]}}{2} .
$$

Then, we can obtain the following theorem.

Theorem 3. When an implementation set $\left(\mathcal{H}_{E}, A_{E}\right.$, $\rho_{E}, U_{S E}$ ) implements $U_{S}$ within error $\delta$, the following inequality holds:

$$
\sqrt{\mathcal{F}_{\delta}^{\mathrm{cost}}\left[U_{S}\right]} \geqslant \frac{\mathcal{A}_{U_{S}}-\mathcal{A}_{U_{S E}}}{\delta}-6 \max \left\{\Delta_{A_{S}}, 2 \mathcal{A}_{U_{S E}}\right\} .
$$

We can obtain the proof of this theorem just by substituting the following inequalities for (42), (43), and $\Delta \leqslant \Delta_{A_{S}}$ in the proof of Theorem 1:

$$
\max \left\{V_{\sigma_{E, \uparrow}}\left(A_{E}\right), V_{\sigma_{E, \downarrow}}\left(A_{E}\right)\right\} \leqslant \delta+2 \max \left\{\Delta_{A_{S}}, 2 \mathcal{A}_{U_{S E}}\right\},
$$

$$
\begin{gathered}
2\left(\mathcal{A}_{U_{S}}-\mathcal{A}_{U_{S E}}\right) \leqslant \Delta+4 \delta\left(\rho_{S, \uparrow+\downarrow}\right) \Delta_{A_{S}}, \\
\Delta \leqslant \Delta_{A_{S}}+\mathcal{A}_{U_{S E}} .
\end{gathered}
$$

We show these inequalities below. The inequality (E5) is obvious. Let us show (E4). We define

$$
\begin{aligned}
& \Delta_{\uparrow}^{S}:=\operatorname{Tr}\left[A_{S}\left(\rho_{S, \uparrow}-\sigma_{S, \uparrow}\right)\right], \\
& \Delta_{\downarrow}^{S}:=\operatorname{Tr}\left[A_{S}\left(\rho_{S, \downarrow}-\rho_{S, \downarrow}^{\prime}\right)\right] .
\end{aligned}
$$

Then, clearly $\left|\Delta_{\uparrow}^{S}-\Delta_{\downarrow}^{S}\right| \leqslant \Delta+2 \mathcal{A}_{U_{S E}}$. In the same manner as the derivation of (B8), we obtain

$$
2 \mathcal{A}_{U_{S}} \leqslant\left|\Delta_{\uparrow}^{S}-\Delta_{\downarrow}^{S}\right|+4 \delta\left(\rho_{S, \uparrow+\downarrow}\right) \Delta_{A_{S}} .
$$

Therefore, we obtain (E4).

Next, we show (E3). We use the following important fact: Let us take an arbitrary positive operator $A$ and arbitrary unitary $U$. When $\|[U, A]\| \leqslant \chi$ holds for a positive real number $\chi$, the following inequality holds for an arbitrary state $\rho$ :

$$
\left|V_{\rho}^{2}(A)-V_{\rho}^{2}\left(U^{\dagger} A U\right)\right| \leqslant \chi\left[2 V_{\rho}(A)+\chi\right],
$$

where $V_{\rho}(A)$ is the standard deviation of $A$ in $\rho$.

Proof of (E9): Because of $\|[A, U]\|=\left\|A-U^{\dagger} A U\right\|$, the Hermitian $X:=A-U^{\dagger} A U$ satisfies $\|X\| \leqslant \chi$. By using $X$, we can express $V_{\rho}^{2}\left(U^{\dagger} A U\right)$ as follows:

$$
\begin{aligned}
V_{\rho}^{2}\left(U^{\dagger} A U\right) & =\left\langle(A-X)^{2}\right\rangle_{\rho}-\langle A-X\rangle_{\rho}^{2} \\
& =V_{\rho}^{2}(A)-2 \operatorname{Cov}_{\rho}(A ; X)+V_{\rho}^{2}(X),
\end{aligned}
$$

where $\operatorname{Cov}_{\rho}(A ; X):=\frac{1}{2} \operatorname{Tr}[\rho(A X+X A)]-\langle A\rangle_{\rho}\langle X\rangle_{\rho}$. Because of $V_{\rho}(X) \leqslant\|X\| \leqslant \chi$ and the quantum correlation coefficient is lower than or equal to 1 , we obtain

$$
\begin{aligned}
\left|V_{\rho}^{2}\left(U^{\dagger} A U\right)-V_{\rho}^{2}(A)\right| & \leqslant 2\left|\operatorname{Cov}_{\rho}(A ; X)\right|+V_{\rho}^{2}(X) \\
& \leqslant 2 V_{\rho}(X) V_{\rho}(A)+V_{\rho}^{2}(X) \\
& \leqslant \chi\left(2 V_{\rho}(A)+\chi\right) .
\end{aligned}
$$

Let us show (E3). By using (E9), we first show that the variances of $A_{S}+A_{E}$ in the initial and the final states are very close to each other. The variance of $A_{S}+A_{E}$ in the initial state is $V_{\rho_{S, i}}^{2}\left(A_{S}\right)+V_{\rho_{E}}^{2}\left(A_{E}\right)$, and corresponds to $V_{\rho}^{2}(A)$ in (E9). The variance of $A_{S}+A_{E}$ in the final state is $V_{\sigma_{S, i}}^{2}\left(A_{S}\right)+V_{\sigma_{E, i}}^{2}\left(A_{E}\right)+2 \operatorname{Cov}_{A_{S}+A_{E}}\left(e^{-i H \tau}\left(\rho_{S, i} \otimes\right.\right.$ $\left.\left.\rho_{E}\right) e^{i H \tau}\right)$, and corresponds to $V_{\rho}^{2}\left(U^{\dagger} A U\right)$ in (E9). Substituting $A_{S}+A_{E}, e^{-i H \tau}$, and $\rho_{S, i} \otimes \rho_{E}$ for $A, U$, and $\rho$ of (E9), 
we obtain

$$
\begin{aligned}
V_{\rho_{S, i}}^{2} & \left(A_{S}\right)+V_{\rho_{E}}^{2}\left(A_{E}\right) \\
\geqslant & V_{\sigma_{S, i}}^{2}\left(A_{S}\right)+V_{\sigma_{E, i}}^{2}\left(A_{E}\right)+2 \operatorname{Cov}_{A_{S}}+A_{E}\left(e^{-i H \tau}\left(\rho_{S, i} \otimes \rho_{E}\right) e^{i H \tau}\right) \\
& -\chi\left(2 \sqrt{V_{\rho_{S, i}}^{2}\left(A_{S}\right)+V_{\rho_{E}}^{2}\left(A_{E}\right)}+\chi\right),
\end{aligned}
$$

where $V_{\rho}\left(A_{S}\right)$ is the standard deviation of the quantity $A$ in $\rho$, and $\operatorname{Cov}_{A_{S}+A_{E}}(\sigma)$ is the covariance of $A$ of the state of $\sigma$ on $S E$. Because $-V_{\sigma_{S, i}}\left(A_{S}\right) V_{\sigma_{E, i}}\left(A_{E}\right) \leqslant \operatorname{Cov}_{A_{S}+A_{E}}\left(e^{-i H \tau}\left(\rho_{S, i} \otimes\right.\right.$ $\left.\rho_{E}\right) e^{i H \tau}$ ) (this is a basic feature of the covariance) and
$V_{\rho}\left(A_{S}\right) \leqslant \Delta_{A_{S}} / 2$ for any $\rho$, we obtain

$$
\begin{aligned}
& V_{\sigma_{E, i}}\left(A_{E}\right)-V_{\sigma_{S, i}}\left(A_{S}\right) \\
& \quad \leqslant \sqrt{V_{\sigma_{S, i}}^{2}\left(A_{S}\right)+V_{\sigma_{E, i}}^{2}\left(A_{E}\right)-2 V_{\sigma_{S, i}}\left(A_{S}\right) V_{\sigma_{E, i}}\left(A_{E}\right)} \\
& \quad \leqslant \sqrt{V_{\rho_{E}}^{2}\left(A_{E}\right)+V_{\rho_{S, i}}^{2}\left(A_{S}\right)+\chi\left(2 \sqrt{V_{\rho_{S, i}}^{2}\left(A_{S}\right)+V_{\rho_{E}}^{2}\left(A_{E}\right)}+\chi\right)} \\
& \quad \leqslant V_{\rho_{E}}\left(A_{E}\right)+1.5 \max \left\{\Delta_{A_{S}}, \chi\right\} .
\end{aligned}
$$

By substituting $2 \mathcal{A}_{U_{S E}}$ for $\chi$, we obtain (E3).
[1] J. Åberg, Catalytic Coherence, Phys. Rev. Lett. 113, 150402 (2014).

[2] E. T. Jaynes and F. W. Cummings, Comparison of Quantum and Semiclassical Radiation Theory with Application to the Beam Maser, Proc. IEEE 51, 89 (1963).

[3] L. Mandel and E. Wolf, Optical Coherence and Quantum Optics (Cambridge University, Cambridge, England, 2008).

[4] R. J. Glauber, Photon Correlations, Phys. Rev. Lett. 10, 84 (1963).

[5] R. J. Glauber, Coherent and Incoherent States of the Radiation Field, Phys. Rev. 131, 2766 (1963).

[6] L. M. K. Vandershypen and I. L. Chuang, NMR techniques for quantum control and computation, Rev. Mod. Phys. 76, 1037 (2004).

[7] T. Rudolph and B. C. Sanders, Requirement of Optical Coherence for Continuous-Variable Quantum Teleportation, Phys. Rev. Lett. 87, 077903 (2001).

[8] S. J. van Enk and C. A. Fuchs, Quantum State of an Ideal Propagating Laser Field, Phys. Rev. Lett. 88, 027902 (2001).

[9] S. D. Bartlett, T. Rudolph, and R. W. Spekkens, Dialogue Concerning Two Views on Quantum Coherence: Factist and Fictionist, Int. J. Quantum. Inform. 4, 17 (2006).

[10] M. P. Woods, R. Silva, and J. Oppenheim, Autonomous Quantum Machines and Finite-Sized Clocks, Ann. Inst. Henri Poincare 20, 125 (2019).

[11] A. S. L. Malabarba, A. J. Short, and P. Kammerlander, Clockdriven Quantum Thermal Engines, New. J. Phys. 17, 045027 (2015).

[12] M. Ozawa, Conservative Quantum Computing, Phys. Rev. Lett. 89, 057902 (2002).

[13] M. Ozawa, Uncertainty principle for quantum instruments and computing, Int. J. Quantum. Inform. 1, 569 (2003).

[14] T. Karasawa and M. Ozawa, Conservation-law-induced quantum limits for physical realizations of the quantum not gate, Phys. Rev. A 75, 032324 (2007).

[15] T. Karasawa, J. Gea-Banacloche, and M. Ozawa, Gate fidelity of arbitrary single-qubit gates constrained by conservation laws, J. Phys. A: Math. Theor. 42, 225303 (2009).

[16] H. Tajima, N. Shiraishi, and K. Saito, Uncertainty Relations in Implementation of Unitary Operations, Phys. Rev. Lett. 121, 110403 (2018).

[17] E. Chitambar and G. Gour, Quantum resource theories, Rev. Mod. Phys. 91, 025001 (2019).

[18] P. Faist and R. Renner, Fundamental Work Cost of Quantum Processes, Phys. Rev. X 8, 021011 (2018).
[19] P. Faist, M. Berta, and F. Brandão, Thermodynamic Capacity of Quantum Processes, Phys. Rev. Lett. 122, 200601 (2019).

[20] Z. Liu and A. Winter, Resource theories of quantum channels and the universal role of resource erasure, arXiv:1904.04201 (2019).

[21] M. G. Díaz, K. Fang, X. Wang, M. Rosati, M. Skotiniotis, J. Calsamiglia, and A. Winter, Using and reusing coherence to realize quantum processes, Quantum 2, 100 (2018).

[22] Y. Liu and X. Yuan, Operational resource theory of quantum channels, Phys. Rev. Research 2, 012035 (2020).

[23] T. Theurer, D. Egloff, L. Zhang, and M. B. Plenio, Quantifying Operations with an Application to Coherence, Phys. Rev. Lett. 122, 190405 (2019).

[24] S. D. Bartlett, T. Rudolph, and R. W. Spekkens, Reference frames, superselection rules, and quantum information, Rev. Mod. Phys. 79, 555 (2007).

[25] G. Gour and R. W. Spekkens, The resource theory of quantum reference frames: Manipulations and monotones, New J. Phys. 10, 033023 (2008).

[26] I. Marvian and R. W. Spekkens, The theory of manipulations of pure state asymmetry: Basic tools and equivalence classes of states under symmetric operations, New J. Phys. 15, 033001 (2013).

[27] I. Marvian and R. W. Spekkens, How to quantify coherence: Distinguishing speakable and unspeakable notions, Phys. Rev. A 94, 052324 (2016).

[28] I. Marvian, Symmetry, Asymmetry and Quantum Information, Ph.D. thesis, 2012.

[29] R. Takagi, Skew informations from an operational view via resource theory of asymmetry Sci. Rep. 9, 14562 (2019).

[30] I. Marvian and R. W. Spekkens, A No-Broadcasting Theorem for Quantum Asymmetry and Coherence and a Trade-Off Relation for Approximate Broadcasting Phys. Rev. Lett. 123, 020404 (2019).

[31] M. Lostaglio and M. P. Müller, Coherence and Asymmetry Cannot be Broadcast, Phys. Rev. Lett. 123, 020403 (2019).

[32] I. Marvian and R. W. Spekkens, Extending Noether's theorem by quantifying the asymmetry of quantum states, Nat. Commun. 5, 3821 (2014).

[33] B. Yadin and V. Vedral, General framework for quantum macroscopicity in terms of coherence, Phys. Rev. A 93, 022122 (2016).

[34] I. Marvian and R. W. Spekkens, Asymmetry properties of pure quantum states, Phys. Rev. A 90, 014102 (2014). 
[35] I. Marvian, Coherence distillation machines are impossible in quantum thermodynamics, Nat Commun 11, 25 (2020).

[36] S. L. Braunstein and C. M. Caves, Statistical Distance and the Geometry of Quantum States, Phys. Rev. Lett. 72, 3439 (1994).

[37] M. Keyl and R. F. Werner, J. Math. Phys. 40, 3283 (1999).

[38] C. H. Bennett, D. P. DiVincenzo, and J. A. Smolin, and W. K. Wootters, Mixed-state entanglement and quantum error correction, Phys. Rev. A 54, 3824 (1996).

[39] G. Tóth and D. Petz, Extremal properties of the variance and the quantum Fisher information, Phys. Rev. A 87, 032324 (2013).

[40] S. Yu, Quantum Fisher Information as the Convex Roof of Variance, arXiv:1302.5311 (2013).

[41] X. N. Feng and L. F. Wei, Quantifying quantum coherence with quantum Fisher information, Sci. Rep. 7, 15492 (2017).

[42] Our resource theoretic formulation can be translated by the formulation using the Hamiltonian. To perform the covariant operation $\Lambda_{S E}$, we introduce another system $E^{\prime}$ and its initial state $\rho_{E^{\prime}}$. The $\rho_{E^{\prime}}$ is symmetric with respect to $A_{E^{\prime}}$, that is the operator of $A$ on $E^{\prime}$. Before the covariant operation, the Hamiltonian of the composite system $S E$ is denoted as $H_{S}+H_{E}+H_{E^{\prime}}$ such that $\left[A_{S}, H_{S}\right]=0,\left[A_{E}, H_{E}\right]=$ 0 and $\left[A_{E^{\prime}}, H_{E^{\prime}}\right]=0$. The covariant operation on $S E$ is written by an interaction Hamiltonian $h_{S E E^{\prime}}$ and an interval $\tau$ as $\Lambda_{S E}\left(\rho_{S} \otimes \rho_{E}\right)=\operatorname{Tr}_{E^{\prime}}\left[U_{S E E^{\prime}}\left(\rho_{S} \otimes \rho_{E} \otimes \rho_{E^{\prime}}\right) U_{S E E^{\prime}}^{\dagger}\right]$, where $U_{S E E^{\prime}}:=e^{-i\left(H_{S}+H_{E}+H_{E^{\prime}}+h_{S E E^{\prime}}\right) \tau}$ and $\left[h_{S E E^{\prime}}, A_{S}+A_{E}+A_{E^{\prime}}\right]=0$. The existence of $A_{E^{\prime}}, \rho_{E^{\prime}}$ and $h_{S E E^{\prime}}$ is guaranteed by the fact that any covariant operation is always realized by a invariant unitary and a symmetric state [see the paragraph above Eq. (5)].

[43] M. Hayashi, Quantum Information Theory: An Introduction (Springer, New York, 2006).

[44] If $\left[U_{S}, A_{S}\right] \neq 0, \quad$ the inequality $\lambda_{\max }\left(\left[U_{S}, A_{S}\right]\right)>$ $0>\lambda_{\min }\left(\left[U_{S}, A_{S}\right]\right)$ must hold, that follows from $\operatorname{Tr}\left[U_{S}^{\dagger} A_{S} U_{S}-A_{S}\right]=0$.

[45] One might wonder why the region $B$ extends to the region $\delta>4 \sqrt{2} \mathcal{A}_{U_{S}} /\left(9 \Delta_{A_{S}}\right)$. This is indicated by Theorem 2 as follows. Theorem 2 guarantees the existence of $\mathcal{I}$ that satisfies $\delta_{\mathcal{I}} \leqslant \delta$ and $\mathcal{F}_{\rho_{E}}\left(A_{E}\right)=\mathcal{F}$ for arbitrary $(\delta, \mathcal{F})$ such that $\delta \leqslant 4 \sqrt{2} \mathcal{A}_{U_{S}} /\left(9 \Delta_{A_{S}}\right)$ and $\mathcal{F} \geqslant \frac{\mathcal{A}_{U_{S}}}{\delta}-\sqrt{2} \Delta_{A_{S}}$. Therefore, for arbitrary $(\delta, \mathcal{F})$ satisfying $\delta=4 \sqrt{2} \mathcal{A}_{U_{S}} /\left(9 \Delta_{A_{S}}\right)$ and $\mathcal{F} \geqslant$ $\frac{\mathcal{A}_{U_{S}}}{\delta}-\sqrt{2} \Delta_{A_{S}}=\frac{17 \Delta_{A_{S}}}{4 \sqrt{2}}$, there exists an $\mathcal{I}$ satisfying $\delta_{\mathcal{I}} \leqslant \delta$ and $\mathcal{F}_{\rho_{E}}\left(A_{E}\right)=\mathcal{F}$. We refer to this $\mathcal{I}$ as $\mathcal{I}_{\mathcal{F}}$. $\mathcal{I}_{\mathcal{F}}$ satisfies $\delta_{\mathcal{I}} \leqslant \delta$ and $\mathcal{F}_{\rho_{E}}\left(A_{E}\right)=\mathcal{F}$ even for $(\delta, \mathcal{F})$ satisfying $\delta>$ $4 \sqrt{2} \mathcal{A}_{U_{S}} /\left(9 \Delta_{A_{S}}\right)$ and $\mathcal{F} \geqslant \frac{\mathcal{A}_{U_{S}}}{\delta}-\sqrt{2} \Delta_{A_{S}}=\frac{17 \Delta_{A_{S}}}{4 \sqrt{2}}$. Therefore, even if $\delta>4 \sqrt{2} \mathcal{A}_{U_{S}} /\left(9 \Delta_{A_{S}}\right)$, then the region $\mathcal{F} \geqslant \frac{17 \Delta_{A_{S}}}{4 \sqrt{2}}$ is included by the region $B$.

[46] If the CPTP map $\mathcal{E}$ satisfies only Condition 1, then there is no tradeoff relation between cost and error. For example, consider $S$ as a qubit, $A_{S}$ as $|1\rangle\langle 1|-| 0\rangle\langle 0|, U_{S}$ as Hadamard gate [it changes $|0\rangle$ to $(|0\rangle+|1\rangle) / \sqrt{2}]$, and $\rho_{S}$ as $|0\rangle\langle 0|$. Let $E, A_{E}$, $\rho_{E}$, and $U_{S E}$ be a qubit, a $|1\rangle\langle 1|-| 0\rangle\langle 0|$, a $(|0\rangle+|1\rangle) / \sqrt{2}$, and a SWAP gate, respectively. Then, although $U_{S}$ is an asymmetric unitary, the finite values of $\mathcal{F}_{\rho_{E}}\left(A_{E}\right)$ and $\delta\left(\rho_{S}\right)=0$ hold simultaneously.

[47] Note that the magnitude of the quantum fluctuation in the external system $E$ is not necessarily greater than the expected value of $A_{E}$. Namely, the initial state $\rho_{E}$ can satisfy $\operatorname{Tr}\left[\rho_{E} A_{E}\right] \gg$ $\mathcal{F}_{\rho_{E}}\left(A_{E}\right)$.

[48] P. Ehrenfest and T. Ehrenfest, The Conceptual Foundations of the Statistical Approach in Mechanics (Dover, New York, 2015).
[49] A. Lenard, Thermodynamical Proof of the Gibbs Formula for Elementary Quantum Systems J. Stat. Phys. 19, 575 (1978).

[50] J. Kurchan, A Quantum Fluctuation Theorem, arXiv:condmat/0007360 (2000).

[51] H. Tasaki, Jarzynski Relations for Quantum Systems and Some Applications, arXiv:cond-mat/0009244 (2000).

[52] U. Seifert, Stochastic Thermodynamics, Fluctuation Theorems, and Molecular Machines, Rep. Prog. Phys. 75, 126001 (2012).

[53] J. J. Park, K. H. Kim, T. Sagawa, and S. W. Kim, Heat Engine Driven by Purely Quantum Information Phys. Rev. Lett. 111, 230402 (2013).

[54] T. N. Ikeda, N. Sakumichi, A. Polkovnikov, and M. Ueda, The second law of thermodynamics under unitary evolution and external operations, Ann. Phys. (NY) 354, 338 (2015).

[55] G. Benenti, G. Casati, K. Saito, and R. S. Whitney, Fundamental Aspects of Steady-state Conversion of Heat to Work at the Nanoscale, Phys. Rep. 694, 1 (2017).

[56] N. Shiraishi and H. Tajima, Efficiency Versus Speed in Quantum Heat Engines: Rigorous Constraint from Lieb-Robinson Bound, Phys. Rev. E 96, 022138 (2017).

[57] J. J. Park, S. W. Kim, and V. Vedral, Fluctuation Theorem for Arbitrary Quantum Bipartite Systems arXiv:1705.01750 (2017).

[58] We can apply our results to the transient case that the final Hamiltonian of $S$ differs from the initial Hamiltonian using the controller trick proposed in [61].

[59] P. Skrzypczyk, A. J. Short, and S. Popescu, Work Extraction and Thermodynamics for Individual Quantum Systems, Nat. Commun. 5, 4185 (2014).

[60] Y. Guryanova, S. Popescu, A. J. Short, R. Silva, and P. Skrzypczyk, Thermodynamics of Quantum Systems with Multiple Conserved Quantities, Nat. Commun. 7, 12049 (2016).

[61] M. Horodecki and J. Oppenheim, Fundamental Limitations for Quantum and Nanoscale Thermodynamics, Nat. Commun. 4, 2059 (2013).

[62] F. Brandão, M. Horodecki, N. Ng, J. Oppenheim, and S. Wehner, The second laws of quantum thermodynamics, Proc. Natl. Acad. Sci. USA 112, 3275 (2015).

[63] H. Tajima, E. Wakakuwa, and T. Ogawa, Large Deviation Implies First and Second Laws of Thermodynamics, arXiv:1611.06614 (2016).

[64] J. Åberg, Truly Work-like Work Extraction via a Single-shot Analysis, Nat. Commun. 4, 1925 (2013).

[65] G. Gour, M. P. Müller, V. Narasimhachar, R. W. Spekkens, and N. Y. Halpern, The Resource Theory of Informational Nonequilibrium in Thermodynamics, Phys. Rep. 583, 1 (2015).

[66] Y. Morikuni, H. Tajima, and N. Hatano, Quantum Jarzynski Equality of Measurement-based Work Extraction, Phys. Rev. E 95, 032147 (2017).

[67] H. Tasaki, Quantum Statistical Mechanical Derivation of the Second Law of Thermodynamics: A Hybrid Setting Approach, Phys. Rev. Lett. 116, 170402 (2016).

[68] M. Hayashi and H. Tajima, Measurement-based Formulation of Quantum Heat Engine, Phys. Rev. A 95, 032132 (2017).

[69] M. Perarnau-Llobet, Elisa Bäumer, K. V. Hovhannisyan, M.Huber, and Antonio Acin, No-Go Theorem for the Characterization of Work Fluctuations in Coherent Quantum Systems, Phys. Rev. Lett. 118, 070601 (2017). 
[70] E. P. Wigner, Die Messung quntenmechanischer Operatoren, Z. Phys. 133, 101 (1952).

[71] H. Araki and M. M. Yanase, Measurement of quantum mechanical operators, Phys. Rev. 120, 622 (1960).

[72] M. M. Yanase, Optimal measuring apparatus, Phys. Rev. 123, 666 (1961)

[73] M. Ozawa, Conservation Laws, Uncertainty Relations, and Quantum Limits of Measurements, Phys. Rev. Lett. 88, 050402 (2002).
[74] H. Tajima and H. Nagaoka, Coherence-variance uncertainty relation and coherence cost for quantum measurement under conservation laws, arXiv:1909.02904 (2019).

[75] Here, we define $\mathcal{K} \models_{\delta} \mathcal{E}_{S} \quad(\mathcal{K}=\mathcal{I}$ or $\mathcal{J})$ as $F\left(\mathcal{E}_{S} \otimes\right.$ $\left.1_{R}\left(\psi_{S R}\right), \Lambda_{S} \otimes 1_{R}\left(\psi_{S R}\right)\right) \geqslant\left(1-\frac{\delta^{2}}{2}\right)$ for arbitrary $\rho_{S} .\left(\psi_{S R}\right.$ is a purification of $\rho_{S}$. This definition is independent of the choice of the purification $\psi_{S R}$.)

[76] C. K. Chui, Concerning rates of convergence of Riemann sums, J. Approximation Theory 4, 279 (1971). 